Refinements of the Weyl tensor classification in five dimensions

This article has been downloaded from IOPscience. Please scroll down to see the full text article.

2012 Class. Quantum Grav. 29155016

(http://iopscience.iop.org/0264-9381/29/15/155016)

View the table of contents for this issue, or go to the journal homepage for more

Download details:

IP Address: 157.193.53.227

The article was downloaded on 16/07/2012 at $16: 13$

Please note that terms and conditions apply. 


\title{
Refinements of the Weyl tensor classification in five dimensions
}

\author{
Alan Coley $^{1}$, Sigbjørn Hervik ${ }^{2}$, Marcello Ortaggio ${ }^{3}$ \\ and Lode Wylleman ${ }^{2,4,5}$ \\ ${ }^{1}$ Department of Mathematics and Statistics, Dalhousie University, Halifax, Nova Scotia \\ B3H 3J5, Canada \\ ${ }^{2}$ Faculty of Science and Technology, University of Stavanger, N-4036 Stavanger, Norway \\ ${ }^{3}$ Institute of Mathematics, Academy of Sciences of the Czech Republic, Žitná 25, \\ 11567 Prague 1, Czech Republic \\ ${ }^{4}$ Department of Mathematical Analysis EA16, Ghent University, 9000 Gent, Belgium \\ ${ }^{5}$ Department of Mathematics, Utrecht University, 3584 CD Utrecht, The Netherlands \\ E-mail: aac@mathstat.dal.ca, sigbjorn.hervik@uis.no, ortaggio@math.cas.cz and \\ lode.wylleman@ugent.be
}

Received 31 March 2012, in final form 21 June 2012

Published 16 July 2012

Online at stacks.iop.org/CQG/29/155016

\begin{abstract}
We refine the null alignment classification of the Weyl tensor of a fivedimensional spacetime. The paper focusses on the algebraically special alignment types N, III, II and D, while types I and $\mathbf{G}$ are briefly discussed. A first refinement is provided by the notion of spin type of the components of highest boost weight. Second, we analyze the Segre types of the Weyl operator acting on bivector space and examine the intersection with the spin type classification. We present a full treatment for types $\mathbf{N}$ and III, and illustrate the classification from different viewpoints (Segre type, rank, spin type) for types II and $\mathbf{D}$, paying particular attention to possible nilpotence, which is a new feature of higher dimensions. We also point out other essential differences with the four-dimensional case. In passing, we exemplify the refined classification by mentioning the special subtypes associated to certain important spacetimes, such as Myers-Perry black holes, black strings, RobinsonTrautman spacetimes and purely electric/magnetic type D spacetimes.
\end{abstract}

PACS numbers: 04.20.Jb, 04.50.-h

\section{Contents}

1. Introduction 2

2. Preliminaries 3

2.1. Boost weight and Weyl tensor constituents 4

2.2. Null alignment type 5

2.3. The Weyl bivector operator 6

0264-9381/12/155016+50\$33.00 C 2012 IOP Publishing Ltd Printed in the UK \& the USA 1 


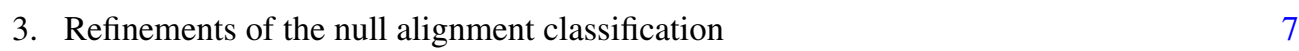

3.1. Spin type refinement $\quad 7$

3.2. Weyl operator refinement 10

4. Type $\mathbf{N} \quad 10$

4.1. Spin types 11

4.2. Weyl operator 11

4.3. Intersection of the two refinements 11

4.4. Comparison with 4D 12

4.5. Discussion 12

5. Type III 12

5.1. Spin types 13

5.2. Weyl operator $\quad 13$

5.3. Comparison with 4D 14

5.4. Discussion 15

6. Type II $\quad 15$

6.1. Spin types 16

6.2. Weyl operator 16

6.3. Type D spacetimes 25

6.4. Comparison with 4D 26

6.5. Discussion 27

7. Types I and $\mathbf{G} \quad 27$

7.1. The 'electric' and 'magnetic' parts of the Weyl operator 28

7.2. Exact purely electric/magnetic solutions 30

8. Conclusions and discussion $\quad 30$

Acknowledgments $\quad 31$

Appendix A. Jordan normal structure and projectors $\quad 31$

Appendix B. Details of the type III Weyl operator classification 33

B.1. Classification based on $\quad 33$

B.2. Subclassification based on $\rho\left(\mathrm{C}^{2}\right) \quad 34$

B.3. Intersection of the two refinements 36

Appendix C. Spin types versus eigenvalues for Weyl type II 39

C.1. Case $\bar{w}=0 \quad 39$

C.2. Case $\bar{w}_{3} \neq 0=\bar{w}_{4}=\bar{w}_{5}$

C.3. Case $\bar{w}_{4}=0 \neq \bar{w}_{3} \bar{w}_{5}$

C.4. General case $\bar{w}_{3} \bar{w}_{4} \bar{w}_{5} \neq 0 \quad 48$

References $\quad 48$

\section{Introduction}

Lorentzian spacetimes with more than four dimensions are of current interest in mathematical physics. It is consequently useful to have higher dimensional generalizations of the classification schemes which have been successfully employed in four dimensions. In particular, the introduction of the alignment theory [1-3], based on the concept of boost weight (abbreviated b.w. throughout this paper), has made it possible to algebraically classify any tensor in a Lorentzian spacetime of arbitrary dimensions by its (null) alignment type, including the classification of the Weyl tensor and the Ricci tensor. To complement this, a higher dimensional generalization of the Newman-Penrose formalism has been presented, which consists of the Bianchi [4] and Ricci identities [5] and of the commutator relations [6] 
written out for a null frame. More recently, the corresponding GHP formalism has also been developed [7].

However, other mathematical tools for the study of higher dimensional Lorentzian spacetimes can also be developed including, e.g., the classification of tensors utilizing bivectors. From this viewpoint, the algebraic (Segre type) classification of the Weyl tensor, considered as a linear operator on bivector space, turns out to be equivalent to the algebraic classification by alignment type in the special case of four dimensions (i.e. the Petrov classification [8]); however, these two classification schemes are non-equivalent in higher dimensions. In particular, the alignment classification is rather course, and developing the algebraic classification of the Weyl bivector operator may lead to a more refined scheme.

For this purpose the bivector formalism in higher dimensional Lorentzian spacetimes was developed in [9]. The Weyl bivector operator was defined in a manner consistent with its b.w. decomposition. Components of fixed b.w. were then characterized in terms of basic constituents which transform under irreducible representations of the spins. This leads to another refinement of the alignment classification, based on geometric relations between the highest b.w. constituents. The types arising will be referred to as spin types.

In this paper we study the general scheme of [9] (and thus the two classification refinements mentioned above, and their interplay) in the case of five-dimensional (5D) Lorentzian spacetimes. These are of particular interest for a number of reasons. First, they provide the simplest arena in which properties of gravity qualitatively differ from the well-known 4D case. Certain important new solutions such as black rings, which are intrinsically higher-dimensional, seem to admit a closed exact form only in five dimensions (see, e.g., [10] and references therein). An alternative spinor classification of the Weyl tensor has also been developed in 5D, and its connection with the b.w. approach has been discussed [11-13]. Since the two classifications are not equivalent, the refinements we propose may also be useful in the spinor classification. Finally, as a peculiar feature of five dimensions (see also [9]), the highest b.w. constituents are represented by square matrices, vectors and a single scalar. In this way both refinements of the 5D Weyl tensor classification can be carried out in a fully explicit manner and its main properties can be easily displayed.

The structure of the paper is as follows. In section 2 we review the algebraic properties of the 5D Weyl tensor (i.e. the constituents, the null alignment types and the Weyl bivector operator). The definitions and the main ideas regarding the spin type and the Weyl operator refinements are presented in section 3. In sections 4-6 we elaborate on both types of refinement and their intersections separately for each of the primary alignment types $\mathbf{N}$, III and II, where special attention is given to the type $\mathbf{D}$ subcase of $\mathbf{I I}$. In section 7 we discuss the split of the Weyl operator in electric and magnetic parts, which is most useful for types $\mathbf{I}$ and $\mathbf{G}$. We conclude in section 8 with a discussion and we make some brief remarks regarding future work. Finally, there are three appendices. Appendix A summarizes some useful basic facts about the Jordan normal form of square matrices. Appendix B provides further details of the Weyl operator classification for type III. The intersection of the spin type and eigenvalue structure classifications in the type II case is exemplified in appendix C.

\section{Preliminaries}

In this section we recapitulate the necessary definitions and results from earlier work, meanwhile introducing the notation and conventions to be used. 


\subsection{Boost weight and Weyl tensor constituents}

Consider a point $p$ of a 5D spacetime $(M, \mathbf{g})$ with Lorentzian metric signature 3 , and assume that the Weyl tensor at $p$ is non-zero. By definition this tensor inherits the basic Riemann tensor symmetries and is moreover traceless:

$$
C_{a b c d}=C_{c d a b}, \quad C_{a b c d}=-C_{b a c d}, \quad C_{a[b c d]}=0, \quad C_{b a d}^{a}=0,
$$

where round (square) brackets denote complete (anti-)symmetrization as usual. Let $\left(\boldsymbol{\ell}, \boldsymbol{n}, \mathbf{m}_{i}, i=3, \ldots, 5\right)$ be a null frame of $T_{p} M$, consisting of two null vectors $\boldsymbol{\ell}$ and $\boldsymbol{n}$, normalized by $l^{a} n_{a}=1$, and three spacelike orthonormal vectors $\mathbf{m}_{i}$, orthogonal to the null vectors $\left(\mathbf{m}_{i}{ }^{a} \mathbf{m}_{j a}=\delta_{i j}, \mathbf{m}_{i}{ }^{a} \boldsymbol{\ell}_{a}=\mathbf{m}_{i}{ }^{a} \boldsymbol{n}_{a}=0\right.$, with $\delta_{i j}$ the Kronecker delta). We take 0 and 1 to be the frame indices corresponding to $\ell$ and $\boldsymbol{n}$, respectively (e.g., $T_{a} l^{a}=T_{0}$ ), whereas $i, j, k, \ldots$ denote spacelike indices, running from 3 to $5 .{ }^{6}$ For a joint notation of the null frame indices we use capital Roman letters $A_{i}$ in the following.

Under a boost in the $(\ell, \boldsymbol{n})$-plane the frame vectors transform according to

$$
\boldsymbol{\ell} \mapsto \lambda \boldsymbol{\ell}, \quad \boldsymbol{n} \mapsto \lambda^{-1} \boldsymbol{n}, \quad \mathbf{m}_{i} \mapsto \mathbf{m}_{i}, \quad \lambda \in \mathbb{R} \backslash\{0\},
$$

such that the components of a rank $p$ tensor $T_{a_{1}, \ldots, a_{p}}$ change as follows:

$$
T_{A_{1}, \ldots, A_{p}} \mapsto \lambda^{b_{A_{1}, \ldots, A_{p}}} T_{A_{1}, \ldots, A_{p}}, \quad b_{A_{1}, \ldots, A_{p}} \equiv \sum_{i=1}^{p}\left(\delta_{A_{i} 0}-\delta_{A_{i} 1}\right),
$$

where $\delta_{A B}$ is the Kronecker-delta symbol. Thus the integer $b_{A_{1}, \ldots, A_{p}}$ is the difference between the numbers of 0 - and 1-indices, and is called the boost weight (b.w.) of the frame component $T_{A_{1}, \ldots, A_{p}}$ (or, rather, of the $p$-tuple $\left(A_{1}, \ldots, A_{p}\right)$ ). For the Weyl tensor, the conditions (1) imply that all components of b.w. \pm 4 or \pm 3 are zero, as well as algebraic relations between the Weyl components of fixed b.w. $(-2 \leqslant$ b.w. $\leqslant 2)[1,4]$ :

b.w. $2: C_{0}{ }^{i} 0 i=0 ; \quad$ b.w. $-2: C_{1}{ }^{i}{ }_{1 i}=0$;

b.w. $1: C_{010 i}=C_{0}{ }^{j}{ }_{i j} ; \quad$ b.w. $-1: C_{101 i}=C_{1}{ }^{j}{ }_{i j}$;

b.w. $0: 2 C_{0(i j) 1}=C_{i}^{k}{ }_{j k}, \quad 2 C_{0[i j] 1}=-C_{01 i j}, \quad 2 C_{0101}=-C^{i j}{ }_{i j}=2 C_{0}{ }^{i}{ }_{1 i}$.

Consider now the spin group, which is isomorphic to $O(3)$ and acts on the null frame according to

$$
\boldsymbol{\ell} \mapsto \boldsymbol{\ell}, \quad \boldsymbol{n} \mapsto \boldsymbol{n}, \quad \mathbf{m}_{j} \mapsto \mathbf{m}_{i} G_{j}^{i}, \quad G_{j}^{i} G_{k}{ }^{j}=\delta_{k}^{i} .
$$

The independent Weyl tensor components of a fixed b.w. $q$ define objects which transform under irreducible representations of the spin group. These objects were presented in [9], for general spacetime dimension $n+2$, and are here referred to as the b.w. $q$ (Weyl) constituents ${ }^{7}$. In particular, the components $C_{i j k l}$ (b.w. 0) and $C_{1 i j k}$ (b.w. -1) are decomposed as follows $(n \geqslant 3)$ :

$$
C_{k l}^{i j} \equiv \bar{H}_{[k l]}^{[i j]}=\bar{C}_{k l}^{i j}+\frac{4}{n-2} \delta_{[k}^{[i} \bar{S}_{l]}^{j]}+\frac{2}{n(n-1)} \bar{R} \delta_{[k}^{[i} \delta_{l]}^{j]}
$$

\footnotetext{
6 We omit the index 2. This is in accordance with [9], but in contrast with [1, 4] and [12], where $(01,234),(12,345)$ are used, respectively.

7 We will use the notation of the Weyl tensor components and constituents used in [9]. It may be useful to compare it with that employed in other works. For negative b.w. components reference [4] defined $2 \Psi_{i j}=C_{1 i 1 j}, 2 \Psi_{j k i}=C_{1 i j k}$ and $\Psi_{i}=-C_{011 i}$, while for zero b.w. components, reference [14] introduced $\Phi_{i j}=C_{0 i 1 j}$, with $\Phi_{i j}^{S}$ and $\Phi_{i j}^{A}$ for its symmetric, respectively, antisymmetric part and $\Phi$ for its trace. These quantities have then appeared in several subsequent papers. A different set of symbols for the full set of Weyl components has been defined in [7].
} 
Table 1. 5D Weyl tensor components and constituents.

\begin{tabular}{lll}
\hline b.w. & Constituents & Weyl tensor components \\
\hline+2 & $\hat{H}_{i j}$ & $C_{0 i 0 j} \equiv \hat{H}_{i j}$ \\
+1 & $\hat{n}_{i j}, \hat{v}_{i}$ & $C_{0 i j k} \equiv \hat{L}_{i[j k]}=2 \delta_{i[j} \hat{v}_{k]}+\hat{n}_{i}^{l} \varepsilon_{l j k}$ \\
& $\bar{S}_{i j}, \bar{w}_{i}, \bar{R}$ & $C_{010 i} \equiv \hat{K}_{i}=-2 \hat{v}_{i}$ \\
& & $\left.C^{i j}{ }_{k l} \equiv \bar{H}^{[i j]}[k l]=4 \delta_{[k}^{[i} \bar{S}^{j]} l\right]$ \\
& & $C_{1 i 0 j} \equiv M_{i j}=-\frac{1}{3} \bar{R} \delta_{[l}^{[i} \delta_{l]}^{j]}$ \\
& & $C_{01 i j} \equiv A_{[i j]}=\varepsilon_{i j k} \bar{w}^{k}$ \\
& & $C_{0101} \equiv \Phi=-\frac{1}{2} \bar{R} \delta_{i j}-\frac{1}{2} \varepsilon_{i j k} \bar{w}^{k}$ \\
-1 & $\check{v}_{i}, \check{n}_{i j}$ & $C_{1 i j k} \equiv \check{L}_{i[j k]}=2 \delta_{i[j} \check{v}_{k]}+\check{n}_{i}{ }^{l} \varepsilon_{l j k}$ \\
& & $C_{101 i} \equiv \check{K}_{i}=-2 \check{v}_{i}$ \\
-2 & $\check{H}_{i j}$ & $C_{1 i 1 j} \equiv \check{H}_{i j}$ \\
\hline
\end{tabular}

$$
C_{1 i j k} \equiv \check{L}_{i[j k]}=2 \delta_{i[j} \check{v}_{k]}+\check{T}_{i j k}, \quad \check{T}_{i k}^{i}=0=\check{T}_{i(j k)} .
$$

Here $\bar{H}_{i j k l}$ symbolizes a $n$-dimensional Riemann-like tensor (i.e. a tensor exhibiting all the properties (1), except for the last one), while $\bar{C}_{i j k l}, \bar{R} \equiv \bar{H}^{i j}{ }_{i j}$ and $\bar{S}_{i j} \equiv \bar{H}^{k}{ }_{i k j}-\frac{1}{n} \bar{R} \delta_{i j}$ stand for the associated Weyl tensor, Ricci scalar and tracefree Ricci tensor, respectively. For the case of five dimensions $(n=3)$ to be treated here, we have that the b.w. 0 Weyl constituent $\bar{C}_{i j k l}$ vanishes identically,

$$
\bar{C}_{i j k l}=0
$$

while the b.w. -1 constituent $\check{T}$ is equivalent to a traceless symmetric matrix $\check{n}$ :

$$
\check{n}_{i j} \equiv \frac{1}{2} \varepsilon^{k l}{ }_{(i} \check{T}_{j) k l} \quad \Leftrightarrow \quad \check{T}^{i}{ }_{j k}=\varepsilon_{j k l} \check{n}^{i l}, \quad \check{n}_{i j}=\check{n}_{(i j)}, \quad \check{n}_{i}^{i}=0,
$$

where $\varepsilon_{i j k}$ denotes the sign of the permutation $(i j k)$ of (345). Analogously for $C_{0 i j k}$, giving rise to b.w. 1 constituents $\hat{v}$ and $\hat{n}$. Regarding the b.w. 0 constituent $A$, defined for general dimensions by $A_{i j} \equiv C_{01 i j}$, we will use one more simplification specific to $n=3$ (not made explicit in [9]): as $A_{i j}$ is antisymmetric in $i j$, we will use its dual vector $\bar{w}$ as the equivalent Weyl constituent:

$$
\bar{w}_{i}=\frac{1}{2} \varepsilon_{i j k} A^{j k} \quad \Leftrightarrow \quad A_{i j}=\varepsilon_{i j k} \bar{w}^{k}
$$

The symbols of the Weyl constituents and their relation with the Weyl components are summarized in table 1 , where the relations (4)-(6) have been implicitly included. The twoindex constituents $\hat{H}, \hat{n}, \bar{S}, \breve{n}$ and $\breve{H}$ are traceless and symmetric $3 \times 3$ matrices; those with one index define $3 \times 1$ column vectors $\hat{v}, \bar{w}$ and $\breve{v}$. Together with $\bar{R}$ they add up to the $25+9+1=35$ independent components of the 5D Weyl tensor.

\subsection{Null alignment type}

Given the null frame $\left(\boldsymbol{\ell}, \boldsymbol{n}, \mathbf{m}_{i}\right)$ of $T_{p} M$, the boost order of a rank $p$ tensor $T_{a_{1}, \ldots, a_{p}}$ with respect to the frame is defined to be the maximal b.w. of its non-vanishing components in the frame decomposition [2]. This integer is invariant under the subgroup Fix $([\ell])$ of Lorentz transformations fixing the null direction $[\ell]{ }^{8}$ It follows that the boost order is a function of $[\ell]$ only, denoted by $b_{T}([\ell])$. For the Weyl tensor $C_{a b c d}$ and for generic $\ell$ we have $b_{C}([\ell])=2$.

8 The subgroup of Fix $([\ell])$, consisting of special Lorentz transformations, is also known as $\operatorname{Sim}(n)$ in $n+2$ dimensions. 
If a null direction $[\ell]$ exists for which $b_{C}([\ell]) \leqslant 1$, it is called a Weyl aligned null direction (WAND) of alignment order $1-b_{C}([\ell])$. A WAND is called single if its alignment order is 0 , and multiple (double, triple, quadruple) if the alignment order is greater than zero $(1,2,3)$. The integer

$$
\zeta \equiv \min _{\ell} b_{C}([\ell])
$$

is a pointwise invariant of $(M, \mathbf{g})$, defining the (Weyl) primary or principal alignment type $2-\zeta$ at $p$; if $\zeta=2,1,0,1$ or -2 this type is still denoted by $\mathbf{G}, \mathbf{I}, \mathbf{I I}, \mathbf{I I I}$ or $\mathbf{N}$, respectively $[1,2] .{ }^{9}$ For types $\mathbf{N}$ and III the quadruple, respectively triple, WAND $[\ell]$ is in fact the unique multiple WAND [1]. ${ }^{10}$ If there is a unique double WAND in the type II case we will denote this by $\mathbf{I}_{0}$; if there are more of them we denote this by $\mathbf{D} \equiv \mathbf{I I}_{i i}$, in accordance with the secondary alignment type notation introduced in [1,2]. In the present paper we will focus on the algebraically special types $\mathbf{N}, \mathbf{I I I}$ and $\mathbf{I I}$ (or $\mathbf{I I}_{0}$ and $\mathbf{D}$ separately) in the Weyl algebraic classification scheme (and where the context is clear, we will refer to these algebraically special types simply by type II or one of its specializations). Similarly, for type I we will write $\mathbf{I}_{0}$ if there is a unique single WAND, and $\mathbf{I}_{i}$ if there more than one. In general, a spacetime admits no WANDs, and we denote the general case by type $\mathbf{G}$.

\subsection{The Weyl bivector operator}

Let $\wedge{ }^{2} T_{p} M$ be the ten-dimensional real vector space of contravariant bivectors (antisymmetric two-tensors $F^{a b}=F^{[a b]}$ ) at $p$. By the first couple of equations in (1), the map

$$
\text { C : } \quad F^{a b} \mapsto \frac{1}{2} C^{a b}{ }_{c d} F^{c d}=\frac{1}{2} F^{c d} C_{c d}{ }^{a b}
$$

determines a linear operator (or endomorphism) on $\wedge^{2} T_{p} M$, which we shall refer to as the Weyl operator.

A null frame of $T_{p} M$

$$
\left(\ell, \boldsymbol{n}, \mathbf{m}_{i}\right),
$$

where $i=3,4,5$, induces a null basis of $\wedge^{2} T_{p} M$ :

$$
\left(\mathbf{U}_{3}, \mathbf{U}_{4}, \mathbf{U}_{5}, \mathbf{W}, \mathbf{W}_{[45]}, \mathbf{W}_{[53]}, \mathbf{W}_{[34]}, \mathbf{V}_{3}, \mathbf{V}_{4}, \mathbf{V}_{5}\right),
$$

consisting of the simple bivectors (in abstract index notation):

$$
\mathbf{U}_{i}^{a b}=\boldsymbol{n}^{[a} \mathbf{m}_{i}^{b]}, \quad \mathbf{W}^{a b}=\boldsymbol{\ell}^{[a} \boldsymbol{n}^{b]}, \quad \mathbf{W}_{[j k]}^{a b}=\mathbf{m}_{j}^{[a} \mathbf{m}_{k}^{b]}, \quad \mathbf{V}_{i}^{a b}=\boldsymbol{\ell}^{[a} \mathbf{m}_{i}^{b]} .
$$

The only non-zero scalar products among these (as induced by the spacetime metric) are given by

$$
2 \mathbf{U}_{i}^{a b} \mathbf{V}_{j a b}=\delta_{i j}, \quad 2 \mathbf{W}^{a b} \mathbf{W}_{a b}=-1, \quad 2 \mathbf{W}_{[j k]}^{a b} \mathbf{W}_{[l m] a b}=\delta_{j l} \delta_{k m}-\delta_{j m} \delta_{k l} .
$$

With the notation of table 1 , the matrix representation of $\mathrm{C}$ with respect to (16)-(17) can be written in a $(3+4+3)$ block form [9]:

$$
\mathcal{C} \equiv\left[\begin{array}{ccc}
M^{t} & \hat{C}_{K} & \hat{H} \\
\check{C}_{K}^{t} & \Omega & \hat{C}_{-K}^{t} \\
\check{H} & \check{C}_{-K} & M
\end{array}\right],
$$

9 This generalizes the Petrov types I, II, D, III and $\mathbf{N}$ from four to higher dimensions. Petrov types II and D together correspond to primary alignment type II. Type $\mathbf{G}$ does not occur in four dimensions, but is the generic situation in higher dimensions [2]. Type $\mathbf{O}$ corresponds to the trivial case $C_{a b c d}=0$, which we have excluded here.

${ }^{10}$ If there was another multiple WAND $\left[\ell^{*}\right]$ we would get the contradiction $C_{a b c d}=0$ by considering components with respect to a null frame $\left(\ell, \ell^{*}, \mathbf{m}_{i}\right)$. 
where

$$
\hat{C}_{ \pm K} \equiv[ \pm \hat{K} \hat{L}], \quad \Omega \equiv\left[\begin{array}{cc}
-\Phi & -A^{t} \\
A & \bar{H}
\end{array}\right], \quad \check{C}_{ \pm K} \equiv[ \pm \check{K} \check{L}] .
$$

Note that, with respect to the given basis (17), the subspaces

$\mathcal{U} \equiv\left\langle\mathbf{U}_{3}, \mathbf{U}_{4}, \mathbf{U}_{5}\right\rangle, \quad \mathcal{W} \equiv\left\langle\mathbf{W}, \mathbf{W}_{[45]}, \mathbf{W}_{[53]}, \mathbf{W}_{[34]}\right\rangle, \quad \mathcal{V} \equiv\left\langle\mathbf{V}_{3}, \mathbf{V}_{4}, \mathbf{V}_{5}\right\rangle$

precisely contain the bivectors of b.w. $-1,0$ and +1 , respectively. If we denote the projection operator onto $\mathcal{X}$ by $p_{\mathcal{X}}$ and the restriction to $\mathcal{X}$ by $\left.\cdot\right|_{\mathcal{X}}$, then the block entries of $C$ are the respective matrix representations of the maps

$$
\begin{aligned}
& \left.\mathrm{M}^{t} \equiv p_{\mathcal{U}} \circ \mathrm{C}\right|_{\mathcal{U}},\left.\quad \hat{\mathrm{C}}_{\mathrm{K}} \equiv p_{\mathcal{U}} \circ \mathrm{C}\right|_{\mathcal{W}},\left.\quad \hat{\mathrm{H}} \equiv p_{\mathcal{U}} \circ \mathrm{C}\right|_{\mathcal{V}} \\
& \left.\check{\mathrm{C}}_{\mathrm{K}}{ }^{t} \equiv p_{\mathcal{W}} \circ \mathrm{C}\right|_{\mathcal{U}},\left.\quad \Omega \equiv p_{\mathcal{W}} \circ \mathrm{C}\right|_{\mathcal{W}},\left.\quad \hat{\mathrm{C}}_{-\mathrm{K}}{ }^{t} \equiv p_{\mathcal{W}} \circ \mathrm{C}\right|_{\mathcal{V}}, \\
& \left.\check{\mathrm{H}} \equiv p_{\mathcal{V}} \circ \mathrm{C}\right|_{\mathcal{U}},\left.\quad \check{\mathrm{C}}_{-\mathrm{K}} \equiv p_{\mathcal{V}} \circ \mathrm{C}\right|_{\mathcal{W}},\left.\quad \mathrm{M} \equiv p_{\mathcal{V}} \circ \mathrm{C}\right|_{\mathcal{V}} .
\end{aligned}
$$

\section{Refinements of the null alignment classification}

\subsection{Spin type refinement}

3.1.1. Weyl spin type of a null direction. The boost order of the Weyl tensor with respect to a null frame $\left(\boldsymbol{\ell}, \boldsymbol{n}, \mathbf{m}_{i}\right)$ is defined to be the maximal b.w. of its non-vanishing components and is a function of $[\ell]$ only, denoted by $b_{C}([\ell])$ (cf section 2.2). The last statement follows by considering the induced action of an element $g \in \operatorname{Fix}([\ell])$ on the leading terms. A more detailed consideration leads to other invariants for this action, and thus to other functions of (that is, properties associated with) $[\ell]$. To this end, we (uniquely) decompose $g$ into a product $S[G] A[\lambda] N[z]$, where $S[G]$ is a spin (7), $A[\lambda]$ a boost (2) and $N(z)$ a null rotation about $[\ell]$, acting on the null frame according to $\left(|z|^{2} \equiv z_{i} z^{i}\right)$

$$
\boldsymbol{\ell} \mapsto \boldsymbol{\ell}, \quad \boldsymbol{n} \mapsto \boldsymbol{n}+z^{j} \mathbf{m}_{j}-\frac{1}{2}|z|^{2} \boldsymbol{\ell}, \quad \mathbf{m}_{i} \mapsto \mathbf{m}_{i}-z_{i} \boldsymbol{\ell} .
$$

In conjunction with the vanishing of all b.w. $>b_{C}([\ell])$ terms, (23) readily implies that $N(z)$ leaves the leading terms (or, equivalently, the b.w. $b_{C}([\ell])$ constituents) invariant [2]. Moreover, $A[\lambda]$ has the effect of simply multiplying the $b_{C}([\ell])$ constituent components by a common factor $\lambda^{b_{C}([\ell])}$. It follows that spin-invariant quantities defined by the b.w. $b_{C}([\ell])$ constituents are properties associated with $[\ell]$. More specifically for 5D Weyl tensors, the spin-irreducible constituents of b.w. $b_{C}([\ell])$ consist of a traceless, symmetric matrix $X$ (for all values of $\left.b_{C}([\ell])\right)$, a column vector $x\left(\right.$ for $\left.-1 \leqslant b_{C}([\ell]) \leqslant 1\right)$ and a scalar $\bar{R}$ (for $b_{C}([\ell])=0$ only). This leads to the notion of spin type of $[\ell]$, as follows.

The matrix $X$ is either zero or (symmetric and thus) diagonalizable by applying spins. By tracelessness this leads to four possible primary spin types of $[\ell]$, symbolized in a Segre-like notation by

$$
\{(000)\}, \quad\{(11) 1\}, \quad\{110\}, \quad\{111\} .
$$

Here a zero (one) indicates a zero (non-zero) eigenvalue, and round brackets indicate equal eigenvalues. Hence, $\{(000)\}$ corresponds to the trivial case $X=0$. For primary spin type $\{(11) 1\}$ there is one non-degenerate eigendirection and an eigenplane orthogonal to it. The last two types are the non-degenerate primary spin types characterized by three different eigenvalues, each with one corresponding eigendirection; if the distinction between zero and non-zero eigenvalues is irrelevant the joint notation $\{111 / 0\}$ will be utilized. 
Table 2. Primary spin types of $[\ell]$ and normal forms for $X\left(X_{3} X_{4} \neq 0\right)$.

\begin{tabular}{lllll}
\hline Primary spin type & $\{(000)\}$ & $\{(11) 1\}$ & $\{110\}$ & $\{111\}$ \\
Normal form of $X$ & 0 & $X_{3} \kappa_{3}$ & $\operatorname{diff}\left[X_{3},-X_{3}\right]$ & $\operatorname{diff}\left[X_{3}, X_{4}\right]$ \\
\hline
\end{tabular}

If we denote the eigenvalues (without multiplicity) by $X_{i}, i=3, \ldots, 5$, we get $X=\operatorname{diag}\left[X_{3}, X_{4}\right] \equiv \operatorname{diag}\left(X_{3}, X_{4},-X_{3}-X_{4}\right)$ after diagonalization. For the non-degenerate primary types $\{111 / 0\}$ we write $X=\operatorname{diff}\left[X_{3}, X_{4}\right]$ to indicate that $X_{i} \neq X_{j}$ for $i \neq j$. We may interchange the $\mathbf{m}_{i}$ s such that $X=\operatorname{diff}\left[X_{3},-X_{3}\right]\left(X_{5}=0\right)$ in the case of spin type $\{110\}$, and $X=X_{3} \kappa_{3}$ in the case of spin type $\{(11) 1\}$, where

$$
\kappa_{3} \equiv \operatorname{diag}\left(1,-\frac{1}{2},-\frac{1}{2}\right) \leftrightarrow X_{4}=X_{5}=-\frac{1}{2} X_{3} .
$$

The possible normal forms for $X$ are summarized in table 2 . Note that for $b_{C}([\ell])= \pm 2$ the case $\{(000)\}$ should be excluded by definition of boost order (cf table 1).

Next, if $-1 \leqslant b_{C}([\ell]) \leqslant 1$ we consider the vector $x$ and first suppose that $X \neq 0$. If $x$ is non-zero, its position relative to the $X$-eigendirections is a Fix $([\ell])$-invariant, and one distinguishes three qualitatively different cases. Together with the possibility $x=0$ we get four secondary spin types of $[\ell]$, which we symbolize by

- 0: $x=0$;

- $\|: x$ is parallel to a non-degenerate eigendirection;

- $\perp: x$ is orthogonal to a non-degenerate eigendirection, and not coinciding with the other two such directions in the case of primary types $\{111 / 0\}$;

- $\mathrm{g}$ : $x$ is in 'general' position; i.e. none of the above hold.

These symbols are added in subscript to the primary spin type to form the (total) spin type of $[\ell]$. For spin type $\{110\}_{\|}$it may be important to indicate whether $x$ is parallel to the 0 -eigendirection or not, and we will symbolize this by $\{110\}_{\| 0}$ and $\{110\}_{\| 1}$, respectively. Likewise, for spin type $\{110\}_{\perp}$ we will write $\{110\}_{\perp 0}$ if $x$ is orthogonal to the 0 -eigendirection, and $\{110\}_{\perp 1}$ if it is not.

In the case $X=0$ the spin type can be $\{(000)\}_{\|}(x \neq 0)$ for $-1 \leqslant b_{C}([\ell]) \leqslant 1$, and $\{(000)\}_{0}[R \neq 0](x=0)$ for $b_{C}[\ell]=0$ (for this case we will add $\bar{R}=0$ or $\bar{R} \neq 0$ between square brackets after the spin type symbol whenever this distinction is important).

Regarding normal forms, it is possible and advantageous to order the $\mathbf{m}_{i} \mathrm{~s}$ such that $x_{4}=0$ (except for spin type $\{111\}_{\mathrm{g}}$ ) and $x_{3} \neq 0$ whenever $x \neq 0$, instead of taking an arrangement where the $X$-normal forms of table 2 are guaranteed. In fact, this has only implications for spin types $\{111\}_{\| 0},\{110\}_{\perp 0}$ and $\{(11) 1\}_{\perp}$, where the normal forms $X=\operatorname{diff}\left[0, X_{4}\right], X=\operatorname{diff}\left[X_{3}, 0\right]$ and, for example, $X=X_{5} \kappa_{5}$ should be taken instead, where

$$
\kappa_{5} \equiv \operatorname{diag}\left(-\frac{1}{2},-\frac{1}{2}, 1\right) \leftrightarrow X_{3}=X_{4}=-\frac{1}{2} X_{5} .
$$

Additionally, we will take $X=X_{5} \kappa_{5}$ for type $\{(11) 1\}_{\mathrm{g}}$ as well. The resulting normal forms for the highest b.w. constituents are summarized in table 3 . For $b_{C}([\ell])= \pm 1$ the case $\{(000)\}_{0}$ should be excluded by definition of boost order.

3.1.2. Weyl spin type at a point. Since on the one hand Weyl-preferred null directions may exist, and since there are only a finite number of possible spin types on the other, it is natural to define the notion of Weyl spin type at a spacetime point $p$. It is possible to distinguish four cases:

(1) If the Weyl tensor is of alignment type $\mathbf{N}, \mathbf{I I I}, \mathbf{I I}_{0}$ or $\mathbf{I}_{0}$, then the WAND of maximal alignment order $1-\zeta$ (cf definition (13)) is unique. 
Table 3. Spin types of $[\ell]$ and normal forms for $X$ and $x\left(-1 \leqslant b_{C}([\ell]) \leqslant 1\right)$. The scalars $x_{i}$ are non-zero. For spin types $\{111\}_{\| 0}$ and $\{110\}_{\perp 0}$ we have $X_{3}=0$, resp. $X_{4}=0$, but in all other cases the scalars $X_{i}$ are non-zero as well.

\begin{tabular}{|c|c|c|c|}
\hline Sec. type & $\begin{array}{c}\{111 / 0\} \\
X=\operatorname{diff}\left[X_{3}, X_{4}\right]\end{array}$ & $\{(11) 1\}$ & $\begin{array}{c}\{(000)\} \\
X=0\end{array}$ \\
\hline 0 & $x=0$ & $X=X_{3} \kappa_{3}$, & $x=0$ \\
\hline$\|$ & $x=\left(x_{3}, 0,0\right)$ & $x=\left(x_{3}, 0,0\right)$ & $x=\left(x_{3}, 0,0\right)$ \\
\hline$\perp$ & $x=\left(x_{3}, 0, x_{5}\right)$ & $x=\left(x_{3}, 0,0\right)$ & - \\
\hline g & $x=\left(x_{3}, x_{4}, x_{5}\right)$ & $x=\left(x_{3}, 0, x_{5}\right)$ & - \\
\hline
\end{tabular}

(2) If the alignment type is $\mathbf{D}$, consider two distinct double WANDs $[\boldsymbol{\ell}] \neq[\boldsymbol{n}]$. With respect to null frames $\left(\boldsymbol{\ell}, \boldsymbol{n}, \mathbf{m}_{i}\right)$ and $\left(\boldsymbol{n}, \boldsymbol{\ell}, \mathbf{m}_{i}\right)$, the b.w. $\zeta=0$ constituents (which are then the only non-zero Weyl components) relate like $(\bar{S}, \bar{w}, \bar{R})$ and $(\bar{S},-\bar{w}, \bar{R})$, and it immediately follows that the spin types of $[\boldsymbol{n}]$ and $[\boldsymbol{\ell}]$ are the same.

(3) If the alignment type is $\mathbf{I}_{i}$, one considers the single WANDs and, for instance, the total ordering

$$
\left(X_{1}, x_{1}\right)<\left(X_{2}, x_{2}\right) \Leftrightarrow X_{1}<X_{2} \quad \text { or } \quad X_{1}=X_{2}, \quad x_{1}<x_{2},
$$

on the set of possible spin types, where

$$
\{(000)\}<\{110\}<\{(11) 1\}<\{111\}, \quad 0<\|<\perp<\text { g. }
$$

(4) If the alignment type is $\mathbf{G}$, one considers all null directions and, for example, the ordering

$$
\{110\}<\{(11) 1\}<\{111\}
$$

on the set of possible spin types.

Definition 3.1. The Weyl spin type at a spacetime point $p$ is defined to be the spin type of any maximally aligned null direction in the case of the algebraically special alignment type II (and its specializations) and alignment type $\boldsymbol{I}_{0}$, and the minimal spin type of the single WANDs [of all null directions] with respect to the ordering (28) [(29)] in the case of alignment type $\boldsymbol{I}_{i}[\boldsymbol{G}]$.

\section{Remark 3.2.}

(1) The notion of spin type, introduced here for a 5D Weyl tensor, may be readily transferred (in principle) to any dimension and any tensor. Regarding the different alignment types of the Weyl tensor in general dimensions, some spin type subcases have already been pointed out in earlier work $[1,9,15]$, which will be commented on later.

(2) The spin type can be used as a classification tool. In particular, we may try to determine all spacetimes with given Ricci-Segre and Weyl alignment-spin types. In this respect, all 5D Einstein spacetimes $\left(R_{a b}=\frac{R}{5} g_{a b}\right)$ of alignment type $\mathbf{D}$ and spin type $\{(11) 1\}_{\|},\{(11) 1\}_{0}$, $\{(000)\}_{\|}$or $\{(000)\}_{0}[\bar{R} \neq 0]$ have been invariantly classified and partially integrated in [16]; the collection of these spin types corresponds to the situation where the Weyl tensor is isotropic in some spacelike plane, in addition to the boost isotropy in any plane spanned by double WANDs. 


\subsection{Weyl operator refinement}

The Weyl operator $\mathrm{C}$ on $\wedge^{2} T_{p} M$ is characterized by the list of elementary divisors

$$
\left(x-\lambda_{i}\right)^{m_{i j}}, \quad i=1, \ldots, r, \quad j=1, \ldots, v_{i},
$$

where the $\lambda_{i}$ are the distinct, possibly complex, eigenvalues of $C$ and $\nu_{i} \equiv \operatorname{dim}\left(E_{\lambda_{i}}\right)$ is the dimension of the $\lambda_{i}$-eigenspace, which equals the number of Jordan blocks corresponding to $\lambda_{i}$ in the Jordan normal form of C. The Segre type of C is the list of the orders $m_{i j}$ where, for fixed $i$, round brackets are used to enclose them in the case where $v_{i}>1$. For instance, [(3211)12] would indicate that there are three distinct eigenvalues, the first one corresponding to four Jordan blocks of dimension 3, 2, 1 and 1, while the other two correspond to one Jordan block each, of dimensions 1 and 2, respectively. The integer $\sum_{1 \leqslant j \leqslant v_{i}} m_{i j}$ (equaling 7, 1 and 2 for the respective eigenvalues in the example) is the dimension of the generalized eigenspace $M^{\lambda_{i}}$ corresponding to $\lambda_{i}$, which is a C-invariant subspace of $\wedge^{2} T_{p} M$. A basis of the latter is built by concatenating $v_{i}$ Jordan normal sequences (JNSs) of the form

$$
\mathbf{F}_{j}\left[m_{i j}\right] \equiv\left(\mathbf{F}_{j}, \mathrm{C}_{\lambda}\left(\mathbf{F}_{j}\right), \ldots, \mathrm{C}_{\lambda}^{m_{i j}-1}\left(\mathbf{F}_{j}\right)\right), \quad \mathrm{C}_{\lambda} \equiv \mathrm{C}-\lambda \operatorname{id}_{\wedge^{2} T_{p} M}
$$

We shall use the notation $W_{k}^{\prime} \lambda_{i}$ for the span of those bivectors $F_{j}$ for which the length $m_{i j}$ of the corresponding JNS equals $k$, and $p_{k}\left(\lambda_{i}\right)$ for the invariant $\operatorname{dim}\left(W_{k}^{\prime} \lambda_{i}\right)$. The concatenation of the $M^{\lambda_{i}}$-bases yields a Jordan normal basis (JNB) realizing the Jordan normal form of C. We refer to appendix A or standard text books on linear algebra (such as [17]) for a further discussion, dealing with vector space endomorphisms in general.

In four dimensions (4D), the Segre type classification of the Weyl bivector operator is fully equivalent to the alignment type classification, and both reduce to the six distinct Petrov types. In higher dimensions, however, a particular alignment type can allow for different Segre types. In the present 5D analysis we shall focus on the algebraically special alignment type II (and its specializations), and regard classification of a certain property of the Weyl operator as a refinement thereof.

We will treat alignment types $\mathbf{N}$ and III in full detail: we shall deduce the possible Segre types, and write down the kernel $\operatorname{Ker}(\mathrm{C})$, image $\operatorname{Im}(\mathrm{C})$ and a JNB of C. As a type N (III) Weyl operator is nilpotent of index 2 (3), it suffices to study the possibilities of $\rho(\mathbf{C})\left(\right.$ and $\left.\rho\left(\mathbf{C}^{2}\right)\right)$, according to formula (A.15); here and henceforth, we denote $\rho(Z)$ as the rank of a matrix/linear operator $Z$. We also present the compatibility of the Segre and spin type classifications.

Regarding type II (covering $\mathbf{I I}_{0}$ and $\mathbf{D}$ ), we shall emphasize the classification based on $\rho(M)$ and $\rho(\Omega)$, and on the potential nilpotence of $\mathrm{C}$. We shall also determine the possible spin types in the case where $M$ is nilpotent and $\Omega$ has a quadruple eigenvalue. The determination of all possible Segre types for a given spin type would involve a straightforward but tedious investigation. Rather, for illustration, we shall present the complete eigenvalue degeneracies for spin types $\{\cdot\}_{0},\{\cdot\}_{\|}$and $\{(11) 1\}_{\perp}$ in appendix C.

\section{Type $\mathbf{N}$}

In general dimensions, a type $\mathbf{N}$ Weyl tensor is characterized by having a quadruple WAND $[\ell]$. With respect to any null frame $\left(\boldsymbol{\ell}, \boldsymbol{n}, \mathbf{m}_{i}\right)$ all components of b.w. greater than $\zeta=-2$ vanish; i.e. only the Weyl constituent $\breve{H}$ is non-zero:

$$
\hat{H}=0, \quad \hat{n}=\hat{v}=0, \quad \bar{S}=\bar{w}=\bar{R}=0, \quad \check{n}=\check{v}=0, \quad \check{H} \neq 0 .
$$

In fact, the argument given in footnote 10 shows that $[\ell]$ is the only WAND. 
Table 4. Type $\mathbf{N}$ Weyl tensors: spin types (columns) for given Segre types (rows). The symbols and $\mathrm{x}$ indicate that the corresponding Segre type is not or is allowed, respectively.

\begin{tabular}{llll}
\hline & $\{110\}$ & $\{(11) 1\}$ & $\{111\}$ \\
\hline$[(2221111)]$ & - & $\mathrm{x}$ & $\mathrm{x}$ \\
{$[(22111111)]$} & $\mathrm{x}$ & - & - \\
\hline
\end{tabular}

\subsection{Spin types}

The allowed spin types of the WAND, and thus of the spacetime, are $\{(11) 1\},\{110\}$ and $\{111\}$ (these were summed up in section 4.5 of [9]). We shall use the normal forms for $X=\check{H}$ of table 2 . By an additional boost-normalization, we could naturally take $\check{H}=\operatorname{diff}[1,-1]$ in the case of spin type $\{110\}$, and $\check{H}_{3}= \pm \kappa_{3}$ in the $\{(11) 1\}$ case (where \pm is the sign of $X_{3}$ ).

\subsection{Weyl operator}

With (31) and the diagonal normal form of $\breve{H}$ the Weyl operator takes the form:

$$
\mathcal{C}=\left[\begin{array}{ccc}
0 & 0 & 0 \\
0 & 0 & 0 \\
\check{H} & 0 & 0
\end{array}\right], \quad \check{H}=\operatorname{diag}\left(\check{H}_{3}, \check{H}_{4}, \check{H}_{5}\right) .
$$

Obviously $\mathcal{C}^{2}=0$; i.e. $\mathrm{C}$ is nilpotent of index 2 such that its only eigenvalue is 0 , and $\mathcal{V} \oplus \mathcal{W} \preceq \operatorname{Ker}(\mathrm{C})$. Also, $\mathrm{C}\left(\mathbf{U}_{i}\right)=\check{H}_{i} \mathbf{V}_{i}$ (no sum over $i$ ), and by $\check{H}_{3} \check{H}_{4} \neq 0$ it follows that $2 \leqslant \rho(\mathrm{C})=\rho(\check{H}) \leqslant 3$, where $\rho(\mathrm{C})=2 \Leftrightarrow \check{H}_{5}=0$. From formula (A.15), with $\lambda_{A}=0, s(0)=2, \mathrm{~N}_{A}=\mathrm{C}$, we get

$$
p_{2}(0)=\rho(\mathrm{C}), \quad p_{1}(0)=10-2 \rho(\mathbf{C}) .
$$

This leads to two possibilities (cf section 3.2 for the notation).

(1) Segre type $[(2221111)] \Leftrightarrow \rho(\mathrm{C})=3 \Leftrightarrow \check{H}_{5} \neq 0$, with

$$
\begin{aligned}
& \operatorname{Im}(\mathbf{C})=\mathcal{V}=\left\langle\mathbf{V}^{3}, \mathbf{V}^{4}, \mathbf{V}^{5}\right\rangle, \quad \operatorname{Ker}(\mathbf{C})=\mathcal{V} \oplus \mathcal{W}, \\
& J N B=\left(\mathbf{U}_{3}[2], \mathbf{U}_{4}[2], \mathbf{U}_{5}[2],[\mathbf{W}],\left[\mathbf{W}_{[45]}\right],\left[\mathbf{W}_{[53]}\right],\left[\mathbf{W}_{[34]}\right]\right),
\end{aligned}
$$

corresponding to $W_{2}^{\prime(0)}=\mathcal{U}$ and $W_{1}^{\prime(0)}=\mathcal{W}$.

(2) Segre type $[(22111111)] \Leftrightarrow \rho(\mathrm{C})=2 \Leftrightarrow \check{H}_{5}=0$, with

$$
\begin{aligned}
& \operatorname{Im}(\mathbf{C})=\left\langle\mathbf{V}^{3}, \mathbf{V}^{4}\right\rangle, \quad \operatorname{Ker}(\mathbf{C})=\left\langle\mathbf{U}^{5}\right\rangle \oplus \mathcal{V} \oplus \mathcal{W}, \\
& J N B=\left(\mathbf{U}_{3}[2], \mathbf{U}_{4}[2],\left[\mathbf{U}_{5}\right],\left[\mathbf{V}_{5}\right],[\mathbf{W}],\left[\mathbf{W}_{[45]}\right],\left[\mathbf{W}_{[53]}\right],\left[\mathbf{W}_{[34]}\right]\right),
\end{aligned}
$$

corresponding to $W_{2}^{\prime(0)}=\left\langle\mathbf{U}^{3}, \mathbf{U}^{4}\right\rangle$ and $W_{1}^{\prime(0)}=\left\langle\mathbf{U}^{5}\right\rangle \oplus\left\langle\mathbf{V}^{5}\right\rangle \oplus \mathcal{W}$.

Here $\mathbf{U}_{i}[2]=\left[\mathbf{U}^{i}, \check{H}_{i} \mathbf{V}_{i}\right]$ for $\check{H}_{i} \neq 0(\operatorname{cf}(30))$.

\subsection{Intersection of the two refinements}

The intersection of the spin type and Segre type classifications is trivial and summarized in table 4: case 1 above covers spin types $\{111\}$ and $\{(11) 1\}$, whereas case 2 corresponds precisely to spin type $\{110\}$. 


\subsection{Comparison with $4 D$}

Consider a 4D spacetime which is of (Weyl-Petrov) type $\mathbf{N}$ at a point. With respect to any null frame $\left(\boldsymbol{\ell}, \boldsymbol{n}, \mathbf{m}_{3}, \mathbf{m}_{4}\right)$ with $[\ell]$ the quadruple, unique WAND, we formally have (31), so that with respect to a bivector frame

$$
\left(\mathbf{U}_{3}, \mathbf{U}_{4}, \mathbf{W}, \mathbf{W}_{[34]}, \mathbf{V}_{3}, \mathbf{V}_{4}\right)
$$

defined as in (17), the Weyl operator $C$ takes the $(2+2+2)$-block form formally equal to (32). Hence $C$ is nilpotent of index 2 . However, $\{11\}$ is the only possible spin type here, where we may take $\check{H}=\operatorname{diag}(1,-1)$ after boost-normalization. Also, $\rho(\mathrm{C})=2$ and the only possible Segre type of $C$ is [(2211)]. On writing

$$
\mathcal{V} \equiv\left\langle\mathbf{V}_{3}, \mathbf{V}_{4}\right\rangle, \quad \mathcal{W} \equiv\left\langle\mathbf{W}, \mathbf{W}_{[34]}\right\rangle, \quad \mathcal{U} \equiv\left\langle\mathbf{U}_{3}, \mathbf{U}_{4}\right\rangle
$$

we have

$$
\begin{aligned}
& \operatorname{Im}(\mathrm{C})=\mathcal{V}, \quad \operatorname{Ker}(\mathrm{C})=\mathcal{V} \oplus \mathcal{W}, \\
& J N B=\left(\left[\mathbf{U}_{3}, \mathbf{V}_{3}\right],\left[\mathbf{U}_{4},-\mathbf{V}_{4}\right],[\mathbf{W}],\left[\mathbf{W}_{[34]}\right]\right)
\end{aligned}
$$

We note that certain four-dimensional results on the Weyl operator (including the rank properties) were given in $[18,19]$.

\subsection{Discussion}

From the above we conclude that for alignment type $\mathbf{N}$ Weyl tensors, the spin type classification refines the Weyl operator Segre type classification; the latter coincides with the classification based on the rank of C; i.e. on the number of non-zero eigenvalues of the matrix $\breve{H}$. Although only the $5 \mathrm{D}$ case has been treated here, it is clear that these statements still hold in $n+2$ spacetime dimensions: for $\rho(\mathrm{C})=m \geqslant 2$ we will have precisely $m$ two-dimensional and $n-2(m-1)$ one-dimensional Jordan blocks, reflected in the Segre type; this Segre type is independent of the degeneracies of non-zero $\breve{H}$-eigenvalues. Note that $m \geqslant 2$ is due to the tracelessness of $\check{H}$; the case $m=2$ (Segre type $[(2211 \cdots 1)]$ ) corresponds precisely to the unique spin type $\{1100 \cdots 0\}$, while for fixed $m>2 \check{H}$-eigenvalue degeneracies become possible and the corresponding Segre type covers several spin types.

In $4 \mathrm{D}$ the matrix $\stackrel{H}{H}$ is two-dimensional and $m=2$ is the only possibility, corresponding to spin type $\{11\}$. Most remarkably, for type $\mathbf{N}$ non-Kundt Einstein spacetimes $\left(R_{a b}=\frac{R}{D} g_{a b}\right)$ in any dimensions $D$, the case $m=2$ is the unique possibility as well, which is due to the compatibility with the Bianchi identities [4, 7]. Some explicit examples have been constructed in [20].

However, such a constraint does not apply to Kundt-Einstein spacetimes. As an illustration, let us recall the homogeneous plane-wave spacetimes,

$$
\mathrm{d} s^{2}=2 \mathrm{~d} u\left(\mathrm{~d} v+a_{i j} x^{i} x^{j} \mathrm{~d} u\right)+\delta_{i j} \mathrm{~d} x^{i} \mathrm{~d} x^{j},
$$

where $a_{i j}$ is a constant matrix. If the matrix $a_{i j}$ is traceless then this is Ricci-flat, otherwise some pure radiation will be present. In both cases this metric is of Weyl type $\mathbf{N}$ and the eigenvalue type of $a_{i j}$ is directly related to the spin type of the Weyl tensor.

\section{Type III}

In general dimensions, a type III Weyl tensor is characterized by having a triple WAND [ $\ell]$. With respect to any null frame $\left(\ell, \boldsymbol{n}, \mathbf{m}_{i}\right)$ all components of b.w. greater than $\zeta=-1$ vanish, whereas those of b.w. -1 are not all zero; in terms of constituents this is

$$
\hat{H}=0, \quad \hat{n}=\hat{v}=0, \quad \bar{S}=\bar{w}=\bar{R}=0, \quad(\check{n}, \check{v}) \neq(0,0) .
$$


The argument given in footnote 10 shows that any other WAND must be single; i.e. [ $\ell]$ is the unique triple WAND and there can be no double WANDs. The existence of a single WAND corresponds to $\check{H}=0$, and is symbolized within the full alignment type notation by III $_{i}[1]$.

\subsection{Spin types}

The allowed spin types of the unique triple WAND, and thus of the spacetime, are the combinations in table 2 , with the exception of $\{(000)\}_{0}$ (which would yield type $\mathbf{N}$ or $\mathbf{O}$ ). The secondary spin type '0' (i.e. $\breve{v}=0$ ) was denoted as type III(a) in [1, 15] and III(A) in [9]. The primary spin types were also mentioned in [9], where $\{(000)\}(\check{n}=0)$ was denoted by III(B).

\subsection{Weyl operator}

From (34), and taking the diagonal normal form for $\check{n}$, the Weyl operator takes the form:

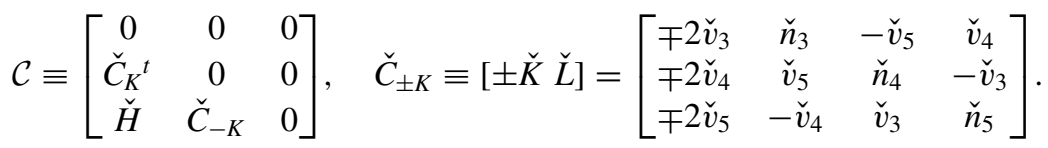

Obviously we have $\mathcal{C}^{3}=0$ and thus 0 is the only eigenvalue, just as for type $\mathbf{N}$. However, contrary to the type $\mathbf{N}$ case, a type III Weyl operator satisfies $\mathcal{C}^{2} \neq 0$. A proof hereof, in general dimensions, was given in [6], lemma 12; however, let us present a shortcut, specifically for five dimensions.

Proposition 5.1. A 5D type III Weyl operator $\mathrm{C}$ is nilpotent of index 3 ; i.e. $\mathrm{C}^{3}=0 \neq \mathrm{C}^{2}$.

Proof. We have

$$
\mathcal{C}^{2} \equiv\left[\begin{array}{ccc}
0 & 0 & 0 \\
0 & 0 & 0 \\
\check{C}_{-K} \cdot \check{C}_{K}^{t} & 0 & 0
\end{array}\right]
$$

where a dot denotes matrix multiplication. Suppose that $\mathcal{C}^{2}=0 \Leftrightarrow \check{C}_{-K} \cdot \check{C}_{K}^{t}=0$. By $\check{C}_{ \pm K}=[ \pm \check{K} \breve{L}]$, this is equivalent to

$$
\check{L} \cdot \check{L}^{t}=\check{K} \cdot \check{K}^{t}
$$

This would imply $\rho(\check{L})=\rho\left(\check{L} \cdot \check{L}^{t}\right)=1$ and hence $\check{L}=a x^{t}$, with $a, x \in \mathbb{R}^{3 \times 1}$. Compatibility with (37) then requires $\breve{L}=\check{K} e^{t}$, with $e$ a unit vector in $\mathbb{R}^{3 \times 1}$. But then $\check{C}_{K}=\check{K}\left[1 e^{t}\right]$ such that $2 \leqslant \rho\left(\check{C}_{K}\right)=1$ : this is a contradiction.

The difference between the indices of nilpotence serves as an easily testable criterion for distinguishing the alignment types III and $\mathbf{N}$.

Next, from (35) it is clear that the order 2 minors ${ }^{11} \check{C}_{K}\left(\begin{array}{ll}i_{1} & i_{2} \\ j_{1} & j_{2}\end{array}\right)$ of $\check{C}_{ \pm K}$ cannot all be zero, as this would lead to $\check{v}=\check{n}=0$ and thus type $\mathbf{N}$. Hence, $\rho\left(\check{C}_{K}\right)=\rho\left(\check{\mathbf{C}}_{-\mathrm{K}}\right)=\rho\left(\check{\mathrm{C}}_{\mathrm{K}}^{t}\right)$ equals 2 or 3 . Moreover, from the structure of (35) we immediately see that $\mathcal{V} \supsetneqq \operatorname{Ker}(\mathrm{C})$ and

$$
4 \leqslant 2 \rho\left(\check{C}_{K}\right) \leqslant \rho(\mathrm{C}) \leqslant 3+\rho\left(\check{C}_{K}\right) \leqslant 6 .
$$

Defining the order 3 minors

$$
d \equiv \operatorname{det}(\check{L})=\check{n}_{3} \check{n}_{4} \check{n}_{5}+\check{n}_{3} \check{v}_{3}^{2}+\check{n}_{4} \check{v}_{4}^{2}+\check{n}_{5} \check{v}_{5}^{2},
$$

${ }^{11}$ An order $p$ minor $M\left(\begin{array}{llll}i_{1} & i_{2} & \cdots & i_{p} \\ j_{1} & j_{2} & \cdots & j_{p}\end{array}\right)$ of a matrix $M \in \mathbb{R}^{m \times n}, 1 \leqslant i_{1}<\cdots<i_{p} \leqslant m, 1 \leqslant j_{1}<\cdots<j_{p} \leqslant n$, is the determinant of the $p \times p$ submatrix $A$ of $M$ with $A_{k l}=M_{i_{k} j_{l}}, 1 \leqslant k, l \leqslant p$. 
Table 5. Type III Weyl bivector operators: summary of the (six) possible Segre types and corresponding conditions on relevant determinants (defined in the text).

\begin{tabular}{llll}
\hline & $\rho\left(\mathrm{C}^{2}\right)=3$ & $\rho\left(\mathrm{C}^{2}\right)=2$ & $\rho\left(\mathrm{C}^{2}\right)=1$ \\
\hline$\rho(\mathrm{C})=6\left(D^{6} \neq 0\right)$ & {$[(3331)]\left(D^{6<} \neq 0\right)$} & {$[(3322)] \quad\left(D^{6<}=0\right)$} & - \\
$\rho(\mathrm{C})=5\left(D^{6}=0 \neq D^{45}\right)$ & - & {$[(33211)]\left(D^{45<} \neq 0\right)$} & {$[(32221)]\left(D^{45<}=0\right)$} \\
$\rho(\mathrm{C})=4\left(D^{6}=0=D^{45}\right)$ & - & {$[(331111)]\left(D^{45<} \neq 0\right)$} & {$[(322111)]\left(D^{45<}=0\right)$} \\
\hline
\end{tabular}

$$
d_{i} \equiv 2\left[\check{v}_{j} \check{v}_{k}\left(\check{n}_{j}-\check{n}_{k}\right)-\check{v}_{i}\left(\check{v}_{3}^{2}+\check{v}_{4}^{2}+\check{v}_{5}^{2}+\check{n}_{j} \check{n}_{k}\right)\right]
$$

we have

$$
\rho(\mathrm{C})=6 \quad \Leftrightarrow \quad \rho\left(C_{K}\right)=3 \quad \Leftrightarrow \quad D^{6} \equiv d^{2}+d_{3}^{2}+d_{4}^{2}+d_{5}^{2} \neq 0 .
$$

Conforming to the canonical forms of table 3 , we take $\check{v}_{3} \neq 0$ whenever $\check{v} \neq 0$, while in the case $\check{v}=0$ we may permute the $\mathbf{m}_{i} \mathrm{~s}$ such that $\check{n}_{3} \check{n}_{4} \neq 0$. Then, the first and second columns of $\breve{C}_{K}{ }^{t}$ are always independent, as well as columns $j$ and $k$ of $\breve{C}_{-K}$, where

$$
\begin{aligned}
& \check{v}=0\left(\check{n}_{3} \check{n}_{4} \neq 0\right): j=2, k=3 ; \\
& \check{v} \neq 0\left(\check{v}_{3} \neq 0\right): j=1, k=4 \text { or } 3 .
\end{aligned}
$$

It is then easily seen that only one order 5 minor is needed to distinguish between $\rho(C)=4$ and $\rho(\mathrm{C})=5$ :

$$
\rho(\mathrm{C})=4 \quad \Leftrightarrow \quad D^{45} \equiv \mathcal{C}\left(\begin{array}{ccccc}
j+3 & k+3 & 8 & 9 & 10 \\
1 & 2 & 3 & j+3 & k+3
\end{array}\right)=0 .
$$

Finally, based on formula (A.15) with $\lambda_{A}=0, s(0)=3, \mathrm{~N}_{A}=\mathrm{C}$, the numbers $p_{i}(0)$ of Jordan blocks of dimension $i$ are determined by $\rho(\mathbf{C})$ and $\rho\left(\mathbf{C}^{2}\right)$ :

$p_{1}(0)=10-2 \rho(\mathrm{C})+\rho\left(\mathrm{C}^{2}\right), \quad p_{2}(0)=\rho(\mathrm{C})-2 \rho\left(\mathrm{C}^{2}\right), \quad p_{3}(0)=\rho\left(\mathrm{C}^{2}\right)$.

For $\rho(\mathbf{C})=6$ we get $2 \leqslant \rho\left(\mathrm{C}^{2}\right) \leqslant 3$ from $p_{1}(0) \geqslant 0$, where

$$
\rho\left(\mathrm{C}^{2}\right)=2 \quad \Leftrightarrow \quad D^{6<} \equiv \operatorname{det}\left(\check{C}_{-K} \cdot C_{K}{ }^{t}\right) \equiv d^{2}-d_{3}^{2}-d_{4}^{2}-d_{5}^{2}=0 .
$$

For $\rho(\mathrm{C})<6$, proposition 5.1 and $p_{2}(0) \geqslant 0$ yield $1 \leqslant \rho\left(\mathrm{C}^{2}\right) \leqslant 2$, where

$$
\begin{aligned}
\rho\left(\mathrm{C}^{2}\right)=1 \Leftrightarrow D^{45<} \equiv & \left(\check{n}_{3}^{2}+\check{v}_{4}^{2}+\check{v}_{5}^{2}-4 \check{v}_{3}^{2}\right)\left(\check{n}_{4}^{2}+\check{v}^{2}-5 \check{v}_{4}^{2}\right) \\
& -\left(\check{v}_{5}\left(\check{n}_{3}-\check{n}_{4}\right)-5 \check{v}_{3} \check{v}_{4}\right)^{2}=0 .
\end{aligned}
$$

This leads to table 5, which summarizes the possible Segre types that arise in the classification based on the Weyl operator geometry in the type III case. For further details (like $\operatorname{Ker}(\mathrm{C}), \operatorname{Im}(\mathrm{C})$ and the determination of JNBs in the different cases) we refer to appendices B.1 and B.2.

Remark 5.2. If the full alignment type is III $_{i}(\check{H}=0$ in $(35))$ we clearly have $\rho(\mathcal{C})=$ $2 \rho\left(\check{C}_{K}\right)=6$ or 4 , i.e. $D^{45}$ vanishes in this case (cf (B.26) and (B.33)).

\subsection{Comparison with $4 D$}

Consider a 4D, alignment (Weyl-Petrov) type III Weyl tensor, and let [ $\ell$ ] be the unique triple WAND. Referring to the decomposition (9) we have $\breve{T}_{i j k}=0(\Leftrightarrow \check{n}=0)$ [9], such that the only possible spin type is $\{00\}_{\|}$. By applying a spin and a boost we may set $\check{v} \equiv\left(\check{v}_{3}, \breve{v}_{4}\right)=(0,1)$, 
while $\check{H}$ can be transformed to zero by a null rotation (23) about $[\ell]$, such that the full alignment type is $\mathbf{I I I}_{i}[8,9]$. In this gauge the Weyl operator $\mathbf{C}$ takes the $(2+2+2)$-block form

$$
\mathcal{C}=\left[\begin{array}{ccc}
0 & 0 & 0 \\
\check{C}_{K}{ }^{t} & 0 & 0 \\
0 & \check{C}_{-K} & 0
\end{array}\right], \quad \check{C}_{K}^{t}=\left[\begin{array}{cc}
0 & 1 \\
1 & 0
\end{array}\right], \quad \check{C}_{-K}=\left[\begin{array}{cc}
0 & -1 \\
1 & 0
\end{array}\right] .
$$

Obviously $C^{3}=0 \neq C^{2}, \rho(C)=2 \rho\left(C^{2}\right)=4$, and the only possible Segre type is [(33)] and

$\operatorname{Im}(\mathrm{C})=\mathcal{W} \oplus \mathcal{V}, \quad \operatorname{Ker}(\mathbf{C})=\mathcal{V}, \quad J N B=\left(\left[\mathbf{U}_{3}, \mathbf{W}_{[34]},-\mathbf{V}_{3}\right],\left[\mathbf{U}_{4}, \mathbf{W}, \mathbf{V}_{4}\right]\right)$

Cf also [18, 19].

\subsection{Discussion}

It is clear that the Weyl operator geometry approach distinguishes between type III and type $\mathbf{N}$ (for this purpose it suffices to consider $\rho(\mathbf{C})$, equivalent to specifying the number of eigenvectors, or simply the index of nilpotence of $\mathrm{C}$ ). However, for type III there exist different spin types that are indistinguishable from the Segre type viewpoint (and vice versa). We refer to appendix B.3 for a full discussion. Table B1 summarizes the relation between the spin type and Segre type refinement schemes for 5D alignment type III Weyl tensors. Just as for type $\mathbf{N}$, a certain Segre type covers several spin types, and different spin types may allow for exactly the same list of Segre types. However, except for spin types $\{(11) 1\}_{0},\{111\}_{0}$ and $\{(000)\}_{\|}$, such a list does not contain a unique element any more (as was the case for type $\mathbf{N}$ ).

Even before studying more general Weyl types (and/or going to higher dimensions), it is thus already clear from the above 5D type III analysis that the two schemes are essentially independent and represent substantial refinements. This is in contrast to the 4D case, where type III exactly corresponds to a Segre type [(33)] Weyl operator and allows for a single spin type $\left(\{00\}_{\|}\right)$; the same happens for the 4D types II and I (see below).

Finally, we mention that some of the discussed features readily generalize to $(n+2)$ dimensional type III Weyl tensors. In particular, the index of nilpotence for $\mathbf{C}$ is still 3 [6] such that formula (44) still holds, with 10 replaced by $(n+2)(n+1) / 2$. Also, $\rho(\mathrm{C}) \leqslant 2 n, \rho\left(\mathrm{C}^{2}\right) \leqslant n$ a priori, but the lower bounds would need a detailed analysis. Constraints on type III Ricciflat spacetimes were derived in [4] using the Bianchi identities. Some examples of type III Ricci-flat/Einstein spacetimes were constructed in [20], where the 'asymptotic' behavior of the Weyl tensor was also discussed.

\section{Type II}

In general dimensions, a (primary) type II Weyl tensor is characterized by having a double WAND $[\ell]$. With respect to any null frame $\left(\ell, \boldsymbol{n}, \mathbf{m}_{i}\right)$ all components of b.w. greater than $\zeta=0$ vanish, whereas those of b.w. 0 are not all zero; in terms of constituents this is:

$$
\hat{H}=0, \quad \hat{n}=\hat{v}=0, \quad(\bar{S}, \bar{w}, \bar{R}) \neq(0,0,0) .
$$

If the (full) type is D, and if $\boldsymbol{n}$ is a second double WAND, then the components of b.w. less than 0 also vanish:

$$
\check{n}=\check{v}=0, \quad \check{H}=0 .
$$




\subsection{Spin types}

The allowed spin types of any double WAND, and thus of the spacetime (cf section 3.1), are the combinations in table 2 , with the exception of $\{(000)\}_{0}[\bar{R}=0]$. In $[1,9,15]$ the secondary spin type '0' (i.e. $\bar{w}=0$ ) was denoted by subtype $\mathbf{I I}(\mathrm{d})$, the primary spin type $\{(000)\}(\bar{S}=0)$ by $\mathbf{I I}(\mathrm{b})$, and the case $\bar{R}=0$ by $\mathbf{I I}(\mathrm{a})$. In general dimensions the subtype $\mathbf{I I}(\mathrm{c})$ is defined by equation (10); however, this is identically satisfied in five dimensions.

\subsection{Weyl operator}

With (47) the block representation of the Weyl operator reduces to

$$
\mathcal{C} \equiv\left[\begin{array}{ccc}
M^{t} & 0 & 0 \\
\check{C}_{K}^{t} & \Omega & 0 \\
\check{H}^{t} & \check{C}_{-K} & M
\end{array}\right] .
$$

The various submatrices are defined in table 1 and (19), and we work with the diagonal normal form for $\bar{S}$, where

$$
\bar{R}_{i} \equiv \bar{S}_{i}+\frac{1}{3} \bar{R}
$$

with $\bar{S}_{i}$ being the diagonal entries (i.e. eigenvalues) of $\bar{S}$. Then the diagonal block entries $M$ and $\Omega$ take the form

$$
M=\left[\begin{array}{ccc}
-\frac{\bar{R}_{3}}{2} & -\frac{\bar{w}_{5}}{2} & \frac{\bar{w}_{4}}{2} \\
\frac{\bar{w}_{5}}{2} & -\frac{\bar{R}_{4}}{2} & -\frac{\bar{w}_{3}}{2} \\
-\frac{\bar{w}_{4}}{2} & \frac{\bar{w}_{3}}{2} & -\frac{\bar{R}_{5}}{2}
\end{array}\right], \quad \Omega=\left[\begin{array}{cccc}
\bar{R} & -\bar{w}_{3} & -\bar{w}_{4} & -\bar{w}_{5} \\
\bar{w}_{3} & \frac{\bar{R}}{2}-\bar{R}_{3} & 0 & 0 \\
\bar{w}_{4} & 0 & \frac{\bar{R}}{2}-\bar{R}_{4} & 0 \\
\bar{w}_{5} & 0 & 0 & \frac{\bar{R}}{2}-\bar{R}_{5} .
\end{array}\right] .
$$

We have

$$
M=0 \quad \Leftrightarrow \quad \Omega=0 \quad \Leftrightarrow \quad \bar{R}_{i}=\bar{w}_{i}=0,
$$

which is not allowed for type II. Also note that $M$, being the sum of an antisymmetric and a diagonalized symmetric part, may represent any $3 \times 3$ matrix and thus may take any Jordan normal form, while this is not the case for $\Omega$.

We shall start by showing that a 5D, primary alignment type II Weyl operator C can be nilpotent, in contrast with the 4D case (cf section 6.4). We will then study its classification from different perspectives: Segre type of $M$ or $\Omega, \rho(M)$ and $\rho(\Omega)$, and spin type. More precisely, we will exemplify the Segre type classifications by treating particular cases and determining all possibilities for the other properties. We then give the conditions for when the ranks $M$ and $\Omega$ attain a given value, and comment on the relation with $\rho(\mathbf{C})$. We also describe the kernels and images of $M$ and $\Omega$, and pay special attention to the type $\mathbf{D}$ subcase. Finally, in appendix $C$ we present the possible eigenvalue degeneracies for spin types $\{\cdot\}_{0},\{\cdot\}_{\|}$and $\{(11) 1\}_{\perp}$, and comment on the more general spin types.

We shall use an extended Segre type notation to indicate the Jordan block dimensions (elementary divisors) of $M, \Omega$ and $\mathrm{C}$ (cf section 3.2). If there is a single eigenvalue zero we indicate this by writing 0 instead of 1 . For a multiple zero eigenvalue, or if we want to emphasize a specific eigenvalue, we write this value followed by the corresponding Jordan block dimensions between round brackets. For a pair of single complex eigenvalues we use $Z \bar{Z}$ or $a \pm \mathrm{i} b(Z \bar{Z})$ (and, e.g., $(Z 21)(\bar{Z} 21)$ or $a+\mathrm{i} b(Z 21), a-\mathrm{i} b(\bar{Z} 21)$ if both correspond 
to one Jordan block of dimension 2 and one of dimension 1). $W \bar{W}$ indicates a different pair of complex eigenvalues (for instance, $\mathrm{C}[\bar{R}(2211), \pm 3 \mathrm{i} \omega(Z \bar{Z}), 10]$ indicates that $\mathrm{C}$ has one eigenvalue $\bar{R}$ with Jordan block dimensions $2,2,1$, 1, one pair of single complex eigenvalues $\pm 3 \mathrm{i} \omega$, one single zero and one single non-zero eigenvalue).

6.2.1. Nilpotence of $\mathrm{C}$. The lower-triangular block structure of a type II Weyl operator (49) is preserved by taking powers $\mathcal{C}^{k}$, which have $\left(\left(M^{k}\right)^{t}, \Omega^{k}, M^{k}\right)$ on the diagonal. Hence, if $M^{k_{0}}=\Omega^{k_{0}}=0$ then $\mathrm{C}^{k_{0}}$ is (at most) a type III operator, and thus $\mathrm{C}^{3 k_{0}}=0$. Therefore, $\mathrm{C}$ is nilpotent if and only if both $\mathrm{M}$ and $\Omega$ are nilpotent.

The characteristic polynomials of $M$ and $\Omega$ are:

$$
\begin{aligned}
& k_{M}(x)=x^{3}-\sigma_{M}^{1} x^{2}+\sigma_{M}^{2} x-\sigma_{M}^{3}, \\
& k_{\Omega}(x)=x^{4}-\sigma_{\Omega}^{1} x^{3}+\sigma_{\Omega}^{2} x^{2}-\sigma_{\Omega}^{3} x+\sigma_{\Omega}^{4},
\end{aligned}
$$

where

$-2 \sigma_{M}^{1}=-2 \operatorname{Tr} M=\sigma_{\Omega}^{1}=\operatorname{Tr} \Omega=\bar{R}=\bar{R}_{3}+\bar{R}_{4}+\bar{R}_{5}$,

$4 \sigma_{M}^{2}=\sigma_{\Omega}^{2}=\bar{R}_{4} \bar{R}_{5}+\bar{R}_{5} \bar{R}_{3}+\bar{R}_{3} \bar{R}_{4}+\bar{w}_{3}^{2}+\bar{w}_{4}^{2}+\bar{w}_{5}^{2}$,

$-8 \sigma_{M}^{3}=-8 \operatorname{det}(M)=\bar{R}_{3} \bar{R}_{4} \bar{R}_{5}+\bar{R}_{3} \bar{w}_{3}^{2}+\bar{R}_{4} \bar{w}_{4}^{2}+\bar{R}_{5} \bar{w}_{5}^{2}$,

$\sigma_{\Omega}^{3}=-8 \sigma_{M}^{3}+\frac{1}{4}\left(\bar{R}-2 \bar{R}_{3}\right)\left(\bar{R}-2 \bar{R}_{4}\right)\left(\bar{R}-2 \bar{R}_{5}\right)$,

$4 \sigma_{\Omega}^{4}=4 \operatorname{det}(\Omega)=\left(\bar{R}-2 \bar{R}_{4}\right)\left(\bar{R}-2 \bar{R}_{5}\right) \bar{w}_{3}^{2}+\left(\bar{R}-2 \bar{R}_{5}\right)\left(\bar{R}-2 \bar{R}_{3}\right) \bar{w}_{4}^{2}$

$$
+\left(\bar{R}-2 \bar{R}_{3}\right)\left(\bar{R}-2 \bar{R}_{4}\right) \bar{w}_{5}^{2}+\frac{1}{4} \bar{R}\left(\bar{R}-2 \bar{R}_{3}\right)\left(\bar{R}-2 \bar{R}_{4}\right)\left(\bar{R}-2 \bar{R}_{5}\right) .
$$

For further purpose, note that these coefficients are linear polynomials in $\bar{w}_{3}^{2}, \bar{w}_{4}^{2}$ and $\bar{w}_{5}^{2}$. The matrices $M$ and $\Omega$ are nilpotent if and only if (abbreviated iff henceforward) all their eigenvalues are zero, which is equivalent to the vanishing of all $\sigma_{M}^{i}$ and $\sigma_{\Omega}^{i}$. From $\sigma_{M}^{1}=\sigma_{\Omega}^{3}+8 \sigma_{M}^{3}=0$ it follows that $\bar{R}_{4}=0=\bar{R}_{3}+\bar{R}_{5}$ (by suitable axis permutation), and then from $\sigma_{M}^{2}=\sigma_{M}^{3}=\sigma_{\Omega}^{4}=0$ we obtain

$$
\bar{R}_{4}=0, \quad \bar{R}_{3}=-\bar{R}_{5} \neq 0, \quad 2 \bar{w}_{3}^{2}=2 \bar{w}_{5}^{2}=\bar{R}_{5}^{2}, \quad \bar{w}_{4}=0 .
$$

Hence, $C$ is nilpotent iff (59) is satisfied. Note that the spin type is then $\{110\}_{\perp 0}[\bar{R}=0]$. We easily find that $M^{3}=0 \neq M^{2}$ and $\Omega^{3}=0 \neq \Omega^{2}$ in this case, such that the Segre types of $M$ and $\Omega$ are $M[0(3)]$ and $\Omega[0(31)]$. Thus, the value of $k_{0}$ above equals 3 , and the operator $C$ will be generically nilpotent of index 9 , but lower indices $\geqslant 3$ can occur. In particular, we clearly have that if a $5 \mathrm{D}$, alignment type $D$ Weyl bivector operator is nilpotent, its index of nilpotence is 3, the Segre type being C[(3331)]. Thus, in this case and from this viewpoint, it is undistinguishable from a generic type III Weyl operator. Let us discuss an easily testable criterion distinguishing between these two situations.

From lemma 8 in [6] we know that any type $\mathbf{D}$ (and, in fact, any primary type II) Weyl tensor (in arbitrary dimensions) must have a non-zero polynomial invariant, whereas all such invariants necessarily vanish in the type III (or $\mathbf{N}$ ) case ${ }^{12}$. The polynomial invariants of the Weyl tensor $C_{a b c d}$ are (by definition) traces of curvature operators built from $C_{a b c d}$ [22]. An example of a first order operator is the aforementioned operator $C$ acting on bivector space.

\footnotetext{
12 Indeed, this is true for any tensor: the alignment theorem and the VSI corollary in [21] state that a tensor has only vanishing polynomial invariants if and only if it is of type III, or simpler.
} 
An example of a second order operator is $C^{a b c d} C_{d e f g}$, acting on the space of contravariant 3tensors. Remarkably, there is another natural first order operator associated to the Weyl tensor, which does not seem to have been considered in the literature before (not even in the 4D case). This is the following operator, acting on the space $S^{2}\left(T_{p} M\right)$ of symmetric two-tensors ${ }^{13}$ :

$$
\mathrm{C}^{s}: Z^{a b}=Z^{(a b)} \mapsto C^{a b}{ }_{c}{ }_{d} Z^{c d} .
$$

Now, both $C$ and $C^{s}$ are nilpotent in the type III (or N) case, and it is easy to verify, in an ad hoc manner in the present $5 \mathrm{D}$ context, that in the proper type II case where $\mathrm{C}$ is nilpotent (i.e. when (59) holds), then $\mathrm{C}^{s}$ cannot be nilpotent at the same time. However, let us first explicitly prove the following more general result, slightly strengthening lemma 8 in [6] and valid in any dimension.

Proposition 6.1. Suppose that a rank 4 tensor $T_{a b c d}$ has symmetries $T_{a b c d}=-T_{\text {bacd }}=T_{c d a b}$, is of primary type II or more special and has nilpotent associated operators $\mathrm{T}$ and $\mathrm{T}^{s}$. Then $T_{a b c d}$ is necessarily of type III or $\mathbf{N}$, i.e. a frame exists in which all components of b.w. 0,1 and 2 vanish.

Note. For a tensor $T_{a b c d}$ satisfying the mentioned symmetries we can indeed define the operators $\mathrm{T}$ and $\mathrm{T}^{s}$ on $\Lambda^{2} T_{p} M$ and $S^{2}\left(T_{p} M\right)$ by formally replacing $C_{a b c d}$ by $T_{a b c d}$ in (14) and (60), respectively. A null frame (15) of $T_{p} M$, where the indices $i$ now run from 3 to the dimension $(n+2)$ of the spacetime, induce a basis (17) of $\Lambda^{2} T_{p} M$ w.r.t. which $\mathrm{T}$ takes a $3 \times 3$ block matrix representation $\mathcal{T}$ as in (18), where we will use the same symbols as there but with a tilde decoration. On the other hand, (15) induces the basis

$$
\begin{array}{ll}
\mathbf{P}^{a b}=\boldsymbol{n}^{a} \boldsymbol{n}^{b}, & \mathbf{Q}_{i}^{a b}=\sqrt{2} \boldsymbol{n}^{(a} \mathbf{m}_{i}^{b)}, \\
\mathbf{O}^{a b}=\sqrt{2} \boldsymbol{n}^{(a} \boldsymbol{\ell}^{b)}, & \left.\mathbf{O}_{i j}^{a b}=\sqrt{2} \mathbf{m}_{i}^{(a} \mathbf{m}_{j}^{b)}(i<j), \quad \mathbf{O}_{i i}^{a b}=\mathbf{m}_{i}^{a} \mathbf{m}_{i}^{b} \text { (no sum over } i\right), \\
\mathbf{R}_{i}^{a b}=\sqrt{2} \boldsymbol{\ell}^{(a} \mathbf{m}_{i}^{b)}, & \mathbf{S}^{a b}=\boldsymbol{\ell}^{a} \boldsymbol{\ell}^{b}
\end{array}
$$

of $S^{2}\left(T_{p} M\right)$. Note that the boost orders of the tensors $\mathbf{P}, \mathbf{Q}_{i},\left(\mathbf{O}, \mathbf{O}_{i j}\right), \mathbf{R}_{i}$ and $\mathbf{S}$ along $\boldsymbol{\ell}$ are $-2,-1,0,1$ and 2, respectively; accordingly, w.r.t. such a frame the operator $\mathrm{T}^{s}$ takes a $5 \times 5$ block matrix representation $\mathcal{T}^{s}$.

Proof. Since $T_{a b c d}$ is of primary type II or more special, a null frame exists in which all its components of strictly positive b.w. (1 or 2) vanish, such that the block matrices $\mathcal{T}$ and $\mathcal{T}^{s}$ are lower triangular. Hence, the nilpotence of $\mathrm{T}$ and $\mathrm{T}^{s}$ is equivalent to the nilpotence of all block entries on the diagonals of $\mathcal{T}$ and $\mathcal{T}^{s}$. Consider first the middle (b.w. 0) block of $\mathcal{T}^{s}$, corresponding to (62). It is easy to check that it is symmetric ${ }^{14}$, and thus should vanish (since a symmetric matrix is diagonalizable). In particular we get $\mathbf{O}^{a b} T_{a c b d} \mathbf{O}^{c d}=-T_{0101}=0$, and $\mathbf{O}^{a b} T_{a c b d} \mathbf{O}_{i j}^{c d}=\tilde{M}_{i j}+\tilde{M}_{j i}=0$ (for all $i$ and $j$ ) implying that the matrix with entries $\tilde{M}_{i j}:=T_{1 i 0 j}$ is antisymmetric. Next, consider the first block on the diagonal of $\mathcal{T}$ and the second block on the diagonal of $\mathcal{T}^{s}$ : these have entries $2\left(\mathbf{V}_{i}\right)_{a b} \mathbf{T}\left(\mathbf{U}_{j}\right)^{a b}=-\tilde{M}_{i j}$ and $\left(\mathbf{R}_{i}\right)_{a b} \mathrm{~T}^{s}\left(\mathbf{Q}_{j}\right)^{a b}=T_{01 i j}-\tilde{M}_{i j}$. Thus they are antisymmetric, whence diagonalizable and 0 . This gives $T_{01 i j}=T_{1 i 0 j}=0$. Finally, the middle block $\tilde{\Omega}$ of $\mathcal{T}$ is now symmetric and thus should also vanish; this gives $T_{i j k l}=0$. Hence, all b.w. 0 components of $T_{a b c d}$ also vanish in the considered frame, and thus the tensor is of type III or $\mathbf{N}$.

In particular we thus conclude that if a proper type II Weyl tensor has a nilpotent Weyl operator $\mathrm{C}$, then the operator $\mathrm{C}^{s}$ has non-zero eigenvalues, and consequently non-zero polynomial invariants (contrary to any type III tensor).

\footnotetext{
${ }^{13}$ It can be shown that the same operator acting on bivector space is equivalent to $\mathrm{C}$.

${ }^{14}$ The $\sqrt{2}$ normalization factors in (62) have been introduced for this reason. Essentially this normalization implies that the dual basis vectors of the (62) frame vectors are the metric dual ones.
} 
6.2.2. Case of a nilpotent M. In order to illustrate the classification of a type II operator C from the viewpoint of the extended Segre type of $M$, we shall determine the possible spin types and the extended Segre types of $\Omega$, in the case of a nilpotent $M$; i.e. when the extended Segre type of $M$ is either $M[0(3)]\left(M^{3}=0 \neq M^{2} \leftrightarrow \rho(M)=2\right)$ or $M[0(21)]$ $\left(M^{2}=0 \neq M \leftrightarrow \rho(M)=1\right)$.

The matrix $M \neq 0$ is nilpotent iff all of the eigenvalues are zero. This happens iff

$$
\sigma_{M}^{1}=0, \quad \sigma_{M}^{2}=0, \quad \sigma_{M}^{3}=0 .
$$

The first equation gives $\bar{R}=0$, so that $\bar{R}_{i}=\bar{S}_{i}$, and the second equation together with (55) implies that spin type $\{(000)\}_{\|}\left(\right.$all $\left.\bar{R}_{i}=0\right)$ is not allowed. Therefore, when M is nilpotent at least two $\bar{R}_{i}$ must be non-zero, and at most two of them can coincide. Hence, without loss of generality, we may assume that $\bar{R}_{3} \neq \bar{R}_{5}$. We can then solve $\sigma_{M}^{2}=\sigma_{M}^{3}=0$ for $\bar{w}_{3}^{2}$ and $\bar{w}_{5}^{2}$, yielding the necessary and sufficient conditions for $\mathrm{M}$ being nilpotent:

$\bar{R}_{4}=-\bar{R}_{3}-\bar{R}_{5}, \quad \bar{w}_{3}^{2}=\frac{\bar{R}_{5}^{3}-\bar{w}_{4}^{2}\left(\bar{R}_{3}+2 \bar{R}_{5}\right)}{\bar{R}_{5}-\bar{R}_{3}}, \quad \bar{w}_{5}^{2}=\frac{\bar{R}_{3}^{3}-\bar{w}_{4}^{2}\left(2 \bar{R}_{3}+\bar{R}_{5}\right)}{\bar{R}_{3}-\bar{R}_{5}}$.

To determine which spin types are allowed we use the normal forms of table 3 with $(X, x)=(\bar{S}, \bar{w})$ and proceed by increasing the number of zero $\bar{w}$-components. The generic spin type is $\{111 / 0\}_{\mathrm{g}}$, corresponding to $\bar{w}_{3} \bar{w}_{4} \bar{w}_{5} \neq 0$. If $\bar{w}_{4}=0 \neq \bar{w}_{3} \bar{w}_{5}$ we generically have spin type $\{111\}_{\perp}$; the special case of spin type $\{(11) 1\}_{g}$ corresponds to the subcase

$$
\bar{R}_{3}=\bar{R}_{4}=-\frac{1}{2} \bar{R}_{5}, \quad \bar{w}_{4}=0, \quad 3 \bar{w}_{3}^{2}=24 \bar{w}_{5}^{2}=2 \bar{R}_{5}^{2}
$$

of (65), while $\{110\}_{\perp}$ leads to $\{110\}_{\perp 0}$ and gives back (59). If $\bar{w}_{4}=\bar{w}_{5}=0 \neq \bar{w}_{3}$, we find from (65):

$$
\bar{R}_{3}=0, \quad \bar{R}_{4}=-\bar{R}_{5} \neq 0, \quad \bar{w}_{4}=\bar{w}_{5}=0, \quad \bar{w}_{3}^{2}=\bar{R}_{5}^{2} .
$$

It can be readily verified that this subcase corresponds precisely to the case where the nilpotence index of $M$ is 2 (Segre type $M[0(21)]$ ), whereas in all other cases this index is 3 (Segre type $M[0(3)])$. Note that (67) is a subcase of spin type $\{110\}_{\| 0}[\bar{R}=0]$. Finally, $\bar{w}=0$ leads to a diagonal, and hence non-nilpotent, $M$.

To determine the possible Segre types of $\Omega$, we first determine the possible multiplicities and nature (real or complex) of the eigenvalues. This is most easily done by means of the discriminant sequence of $(53)$ [23, 24]. Given (65) the coefficients (54)-(58) reduce to

$$
\begin{aligned}
\sigma_{1}^{\Omega} & =\sigma_{2}^{\Omega}=0, \\
\sigma_{3}^{\Omega} & =-2 \bar{R}_{3} \bar{R}_{4} \bar{R}_{5}, \\
\sigma_{4}^{\Omega} & =\left(\bar{R}_{3}-\bar{R}_{4}\right)\left(\bar{R}_{5}-\bar{R}_{4}\right) \bar{w}_{4}^{2}-\left(\bar{R}_{3}^{2}+\bar{R}_{5}^{2}\right) \bar{R}_{4}^{2},
\end{aligned}
$$

where $\bar{R}_{4}=-\bar{R}_{3}-\bar{R}_{5}$, and the discriminant sequence list becomes

$$
\left[4,0,-36\left(\sigma_{3}^{\Omega}\right)^{2}, D_{4}\right], \quad D_{4}=256\left(\sigma_{4}^{\Omega}\right)^{3}-27\left(\sigma_{3}^{\Omega}\right)^{4} \text {. }
$$

Here $D_{4}$ is the classical discriminant of the degree 4 polynomial $k_{\Omega}(x)$ (given (68)) which vanishes iff the latter has multiple roots. If $\sigma_{3}^{\Omega}=\sigma_{4}^{\Omega}=0$ (i.e. 0 is a multiple root) we are in the case (59) of a nilpotent $C$. In all other cases it follows that the so-called revised sign list (see $[23,24]$ ) must have at least one sign switch, such that $\Omega$ has at least one pair of complex eigenvalues. If $D_{4}>0$ there are two (differing) complex pairs (Segre type $\Omega[Z \bar{Z} W \bar{W}]$ ), while for $D_{4} \leqslant 0$ there are two additional real eigenvalues, which coincide iff $D_{4}=0$. In this last case one can further show that this real (non-zero) eigenvalue must correspond to a two-

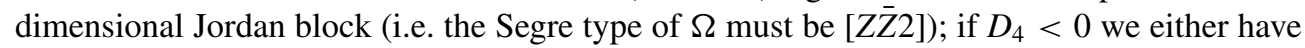


Table 6. Type II Weyl tensors: allowed spin types, and corresponding Segre types and ranks of $M$ and $\Omega$, in the case of a nilpotent matrix $M$. Spin types $\{110\}_{\perp 0},\{(11) 1\}_{\mathrm{g}}$ and $\{110\}_{\| 0}$ correspond to the special cases (59), (66) and (67) of the characterizing equations (65), respectively. In all cases $\bar{R}=0$.

\begin{tabular}{lllll}
\hline Spin types & Segre type of $M$ & $\rho(M)$ & Segre type of $\Omega$ & $\rho(\Omega)$ \\
\hline$\{110\}_{\| 0}$ & {$[0(21)]$} & 1 & {$[Z \bar{Z} 11]$} & 4 \\
$\{110\}_{\perp 0}$ & {$[0(3)]$} & 2 & {$[0(31)]$} & 2 \\
$\{(11) 1\}_{\mathrm{g}},\{111\}_{\perp},\{110\}_{\mathrm{g}}$ & {$[0(3)]$} & 2 & {$[Z \bar{Z} 11]$} & 4 \\
$\{111\}_{\mathrm{g}}$ & {$[0(3)]$} & 2 & {$[Z \bar{Z} 11],[Z \bar{Z} W \bar{W}],[Z \bar{Z} 2]$} & 4 \\
& & & {$[Z \bar{Z} 10]$} & 3 \\
\hline
\end{tabular}

[ZZ̄11] $\left(\sigma_{4}^{\Omega} \neq 0\right)$ or $[Z \bar{Z} 10]\left(\sigma_{4}^{\Omega}=0 \neq \sigma_{3}^{\Omega}\right)$. In the latter case we have $\rho(\Omega)=3$, while $\rho(\Omega)=2$ in the nilpotent case (59); in all other cases we have $\rho(\Omega)=4$.

Regarding the compatibility of the spin types and the Segre types of $\Omega$, we first observe from (71) that $D_{4}<0$ is implied by $\sigma_{4}^{\Omega}<0$. Primary spin type $\{110\}$ is equivalent to $\sigma_{3}^{\Omega}=0$ (see (69)), and we may take $\bar{R}_{4}=\bar{R}_{3}+\bar{R}_{5}=0$ by permuting the axes; moreover, if $\bar{w}_{4} \neq 0$ we then see from (70) that $\sigma_{4}^{\Omega}<0$. The case $\bar{w}_{4}=0 \neq \bar{R}_{4}$ immediately yields $\sigma_{4}^{\Omega}<0$ as well. It follows that $D_{4} \geqslant 0$ is only consistent with the most generic spin type $\{111\}_{\mathrm{g}}$ and the spin type $\{110\}_{\perp 0}\left(\bar{w}_{4}=0=\bar{R}_{4}\right)$ corresponding to nilpotent C; in all other case we have $D_{4}<0$ and thus Segre type $\Omega[Z \bar{Z} 11]$.

A summary of the allowed spin types and Segre types of $M$ and $\Omega$, and their compatibility, in the case of a nilpotent $M$ is given in table 6 .

6.2.3. Case of a quadruple $\Omega$-eigenvalue. Given the analysis of the previous paragraph, we now illustrate the classification of a type $\mathbf{I I}$ operator $\mathbf{C}$ from the viewpoint of the extended Segre type of $\Omega$, by working out the case where $\Omega$ has a quadruple eigenvalue $x_{0}$. This comprises the subcase $x_{0}=0$ of a nilpotent $\Omega$. Strikingly, we will see that the spin type classification forms a pure refinement of the $\Omega$-Segre type classification (in the sense that a certain spin type is compatible with at most one $\Omega$-Segre type) and that the extended Segre type of $M$ is almost always $M[Z \bar{Z} 1]$.

The characteristic polynomial $k_{\Omega}(x)$ of the matrix $\Omega$ has a quadruple root, namely

$$
x_{0}=\frac{1}{4} \sigma_{1}^{\Omega}=\frac{1}{4} \bar{R},
$$

if and only if

$$
\sigma_{2}^{\Omega}=\frac{3}{8} \bar{R}^{2}, \quad \sigma_{3}^{\Omega}=\frac{1}{16} \bar{R}^{3}, \quad \sigma_{4}^{\Omega}=\frac{1}{256} \bar{R}^{4} .
$$

By (54), (55) and (57) it follows that the $\sigma_{i}^{M}$ can be written in terms of the $\bar{R}_{i}$ only:

$$
\sigma_{1}^{M}=-\frac{1}{2} \bar{R}, \quad \sigma_{2}^{M}=\frac{3}{32} \bar{R}^{2}, \quad \sigma_{3}^{M}=\frac{1}{32}\left(F-\frac{1}{4} \bar{R}^{3}\right),
$$

where

$$
F \equiv\left(\bar{R}-2 \bar{R}_{3}\right)\left(\bar{R}-2 \bar{R}_{4}\right)\left(\bar{R}-2 \bar{R}_{5}\right), \quad \bar{R}=\bar{R}_{3}+\bar{R}_{4}+\bar{R}_{5} .
$$

Therefore, the cases where $k_{M}(x)$ has a root 0 or $x_{0}$ respectively correspond to

$$
F=\frac{1}{4} \bar{R}^{3}, \quad F=\frac{5}{2} \bar{R}^{3},
$$

and they occur simultaneously iff $\mathrm{C}$ is nilpotent. Also, the discriminant sequence of a general degree 3 polynomial (52), with arbitrary $\sigma_{M}^{i}$, is

$$
\left[3, F_{3}, D_{3}\right], \quad F_{3} \equiv 2\left(\sigma_{1}^{M}\right)^{2}-6 \sigma_{2}^{M}, \quad 27 D_{3} \equiv-\left[27 \sigma_{3}^{M}-\sigma_{M}^{1}\left(9 \sigma_{2}^{M}-2\left(\sigma_{M}^{1}\right)^{2}\right)\right]^{2}+4 F_{3}^{3} .
$$


Table 7. Type II Weyl tensors: allowed spin types, and corresponding Segre types and ranks of $\Omega$ and $M$, in the case where $\Omega$ has a quadruple eigenvalue. The second row corresponds the case of a nilpotent $\mathrm{C}$.

\begin{tabular}{lllll}
\hline Spin types & Segre type of $\Omega$ & $\rho(\Omega)$ & Segre type of $M$ & $\rho(M)$ \\
\hline$\{(11) 1\}_{\|}[\bar{R} \neq 0]$ & {$[(211)]$} & 4 & {$[Z \bar{Z} 1]$} & 3 \\
$\{110\}_{\perp 0}[\bar{R}=0]$ & {$[0(31)]$} & 2 & {$[0(3)]$} & 2 \\
$\{111\}_{\perp}[\bar{R} \neq 0]$ & {$[(31)]$} & 4 & {$[Z \bar{Z} 1]$} & 3 \\
$\{111\}_{\mathrm{g}}[\bar{R}=0]$ & {$[0(4)]$} & 3 & {$[Z \bar{Z} 1]$} & 3 \\
$\{110\}_{\mathrm{g}}[\bar{R} \neq 0]$ & {$[4]$} & 4 & {$[Z \bar{Z} 1]$} & 3 \\
$\{111\}_{\mathrm{g}}[\bar{R} \neq 0]$ & {$[4]$} & 4 & {$[Z \bar{Z} 1],\left[Z \bar{Z} 1_{c}\right]$} & 3 \\
& & & {$[Z \bar{Z} 0]$} & 2 \\
\hline
\end{tabular}

In the considered situation of a type II Weyl operator with a quadruple $\Omega$-eigenvalue, (54)-(55) and the first equation of (73) imply $F_{3}=-\frac{1}{16} \bar{R}^{2} \leqslant 0$, such that $D_{3} \leqslant 0$ from (77), where $F_{3}<0 \Rightarrow D_{3}<0$. It follows that either $\mathrm{C}$ is nilpotent or $k_{M}(x)$ has a pair of complex roots. The nilpotent case is characterized here by $\bar{R}=\bar{R}_{3} \bar{R}_{4} \bar{R}_{5}=0$. Regarding the latter case we will indicate the respective subcases in (76) by $M[Z \bar{Z} 0]$ and $M\left[Z \bar{Z} 1_{c}\right]$, and otherwise write $M[Z \bar{Z} 1]$.

Just as in the case of a nilpotent $M$, we can show that $\bar{w}=0$ and spin type $\{(000)\}_{\|}$are not allowed. The assumptions of $\bar{w}_{3} \neq 0=\bar{w}_{4}=\bar{w}_{5}$ or primary spin type $\{(11) 1\}$ both lead to the case (up to permutation of the axes):

$$
\bar{w}_{4}=\bar{w}_{5}=0, \quad \frac{1}{2} \bar{R}_{3}=\bar{R}_{4}=\bar{R}_{5}=\bar{w}_{3} \quad\left(x_{0}=\bar{w}_{3}\right),
$$

which is a subcase of spin type $\{(11) 1\}_{\|}[\bar{R} \neq 0]$. It can be verified that this is the only case where $\Omega$ has Jordan blocks of dimension at most 2, and that we have extended Segre types $\Omega\left[\bar{w}_{3}(211)\right]$ and $M\left[-\frac{1 \pm i}{2}(Z \bar{Z}),-\bar{w}_{3}(1)\right]$ and thus $\mathrm{C}[(Z Z)(\bar{Z} \bar{Z})(11)(1111)]$. In all other cases, given (55), (57) and (58), (73) can be solved for the $\bar{w}_{i}^{2}$, yielding:

$\bar{w}_{3}^{2}=\frac{\left(\bar{R}_{3}-\frac{1}{4} \bar{R}\right)^{4}}{\left(\bar{R}_{3}-\bar{R}_{4}\right)\left(\bar{R}_{3}-\bar{R}_{5}\right)}, \quad \bar{w}_{4}^{2}=\frac{\left(\bar{R}_{4}-\frac{1}{4} \bar{R}\right)^{4}}{\left(\bar{R}_{4}-\bar{R}_{5}\right)\left(\bar{R}_{4}-\bar{R}_{3}\right)}, \quad \bar{w}_{5}^{2}=\frac{\left(\bar{R}_{5}-\frac{1}{4} \bar{R}\right)^{4}}{\left(\bar{R}_{5}-\bar{R}_{3}\right)\left(\bar{R}_{5}-\bar{R}_{4}\right)}$.

By substituting this into $\left(\Omega-\frac{1}{4} \bar{R}\right)^{3}$ we find that Segre type $\left.\Omega[(31)]\right)$ corresponds precisely to spin type $\{111 / 0\}_{\perp}$ (i.e. the situation where exactly one $\bar{w}$-component vanishes); in agreement with table 3 this is for

$$
\bar{w}_{4}=0 \neq \bar{w}_{3} \bar{w}_{5} \quad \Leftrightarrow \quad 4 \bar{R}_{4}-\bar{R}=0 \neq\left(4 \bar{R}_{3}-\bar{R}\right)\left(4 \bar{R}_{5}-\bar{R}\right) .
$$

The case $\bar{R}=\bar{R}_{4}=0$ is equivalent to spin type $\{110\}_{\perp 0}$ and gives the case (59) of nilpotent C; for $\bar{R} \neq 0$ one necessarily has spin type $\{111\}_{\perp}$ (i.e. $\{110\}_{\perp 1}$ is not allowed) and extended Segre type $M[Z \bar{Z} 1]$ (i.e. (80) is incompatible with (75)-(76)). Finally, the Segre type $\Omega[4]$ allows for the spin types $\{110\}_{\mathrm{g}}[\bar{R} \neq 0],\{111\}_{\mathrm{g}}[\bar{R}=0]$ and $\{111\}_{\mathrm{g}}$, where the second one gives a nilpotent $\Omega$ and only the last one is compatible with either of the equations in (76).

A summary of the allowed spin types and Segre types of $\Omega$ and $M$, and their compatibility, in the case of a quadruple $\Omega$-eigenvalue is given in table 7 .

6.2.4. Classification based on $\rho(M)$ and $\rho(\Omega)$. The classification by the ranks of $M$ and $\Omega$, and their intersection, gives a rather course subclassification of an alignment type II Weyl tensor, but in combination with the determination of the (kernel and) image of $\mathrm{C}$ may be useful for particular purposes. 
For example, the image has a particular implication for the holonomy group of the spacetime under consideration. The infinitesimal generators of the holonomy group are spanned by:

$$
R_{a b c d} X^{c} Y^{d}, \quad R_{a b c d ; e_{1}} X^{c} Y^{d} Z^{e_{1}}, \quad \ldots
$$

for all vectors $X, Y, Z, \ldots \in T_{p} M$, through the isomorphism $\bar{\imath}: \wedge^{2} T_{p}^{*} M \mapsto \mathfrak{o}(1, n-1)$, where $\mathfrak{o}(1, n-1)$ is the Lie algebra of the Lorentz group. The map $\bar{\imath}$ is explicitly given by raising an index: $X_{a b} \mapsto X_{b}^{a}$. Similarly, by lowering an index we have an isomorphism $\iota: \wedge^{2} T_{p} M \mapsto \mathfrak{o}(1, n-1)$. In particular, this implies that the image of the Riemann bivector operator generates a vector subspace of $\mathfrak{h o l}$ (the Lie algebra of the holonomy group); i.e.

$$
\iota(\operatorname{Im}(\mathrm{R})) \subset \mathfrak{h o l} .
$$

In the case of a Ricci-flat spacetime, this implies that $\iota(\operatorname{Im}(C)) \subset \mathfrak{h o l}$, and thus we can obtain a minimal dimension for the holonomy algebra by considering the rank of $C$. Indeed, if the $\iota(\operatorname{Im}(\mathrm{C}))$ does not close as a Lie algebra, we can consider the algebra it generates (which must also be in $\mathfrak{h o l}$ ). For example, since the dimension of the Lorentz group is 10, we immediately get that the vacuum cases where $\rho(\mathrm{C})=10$ generate the whole Lorentz group (=holonomy group). Indeed, since there are no nine- or eight-dimensional proper subgroups of the Lorentz group, we also have that $\rho(C) \geqslant 8$ must have the full Lorentz group as its holonomy group. Thus to conclude, the image $\operatorname{Im}(C)$ generates a subalgebra of the infinitesimal holonomy algebra. The allowed spin types in each case can be computed.

The generic case, of course, corresponds to having $\rho(M)=3$ and $\rho(\Omega)=4$. We will now work out all possible special cases where lower rank combinations are allowed. From (51) we have $\rho(M) \neq 0 \neq \rho(\Omega)$ and cases of zero-rank matrices can thus be ruled out from the start. Moreover, by considering the order 2 minors of $\Omega$ it is easily shown that $\rho(\Omega)=1$ is not allowed either. As a final preliminary remark we mention that $\rho(\Omega)=2$ (all order $3 \Omega$-minors being zero) leads to the two cases (87) and (88) below, for which $\rho(M)=2$.

We now work out the classification based on $\rho(M)$, and its intersection with that of $\rho(\Omega)$. $\rho(M)=3$. This is the case of a generic matrix $M$, corresponding to

$$
-8 \operatorname{det}(M)=\bar{R}_{3} \bar{R}_{4} \bar{R}_{5}+\bar{R}_{3} \bar{w}_{3}^{2}+\bar{R}_{4} \bar{w}_{4}^{2}+\bar{R}_{5} \bar{w}_{5}^{2} \neq 0 .
$$

If $\Omega$ is also generic then $\rho(\Omega)=4$. The case $\rho(\Omega)<4$ necessarily gives $\rho(\Omega)=3$ by the above remark. This happens if $\operatorname{det}(\Omega)=0$ (i.e. if (58) vanishes while (81) is valid), and occurs for either

$$
\begin{aligned}
& w_{3}^{2}=\frac{\bar{R}_{4}+\bar{R}_{5}-\bar{R}_{3}}{4\left(\bar{R}_{4}-\bar{R}_{5}-\bar{R}_{3}\right)\left(\bar{R}_{4}-\bar{R}_{5}+\bar{R}_{3}\right)} \mathcal{P} \\
& \mathcal{P}=\bar{R}_{3}\left[\bar{R}_{3}^{2}+\bar{R}_{3}\left(\bar{R}_{4}+\bar{R}_{5}\right)-\left(\bar{R}_{4}-\bar{R}_{5}\right)^{2}+4\left(\bar{w}_{3}^{2}+\bar{w}_{4}^{2}\right)\right] \\
& \quad-\bar{R}_{5}\left[\bar{R}_{5}^{2}-\bar{R}_{4} \bar{R}_{5}-\bar{R}_{4}^{2}+4\left(\bar{w}_{4}^{2}-\bar{w}_{5}^{2}\right)\right]-\bar{R}_{4}\left[\bar{R}_{4}^{2}-4\left(\bar{w}_{4}^{2}-\bar{w}_{5}^{2}\right)\right],
\end{aligned}
$$

or

$$
\bar{R}_{3}=0=\bar{R}_{4}-\bar{R}_{5}
$$

In the latter case $(81)$ reduces to $\bar{R}_{5}\left(\bar{w}_{4}^{2}+\bar{w}_{5}^{2}\right) \neq 0$. It is understood that other cases can be obtained from these two by simple axis-permutations (hereafter we will not mention such possibilities any further). The case of nilpotent $\Omega$, corresponding to (79) with $\bar{R}=0 \neq \bar{R}_{3} \bar{R}_{4} \bar{R}_{5}$, is a subcase of (82) (cf also table 7).

$\rho(M)=1$. This is the case where all order 2 minors of $M$ vanish, which happens iff

$$
\bar{R}_{3}=0, \quad \bar{w}_{4}=\bar{w}_{5}=0, \quad \bar{w}_{3}^{2}=-\bar{R}_{4} \bar{R}_{5}, \quad\left(\bar{R}_{4}, \bar{R}_{5}\right) \neq(0,0) .
$$


We have $\sigma_{3}^{M}=\sigma_{2}^{M}=0$, and generically the zero- $M$ eigenvalue is double $\left(\sigma_{1}^{M} \sim \bar{R} \neq 0\right)$. It becomes triple (i.e. M is nilpotent of index 2) for $\bar{R}=\bar{R}_{4}+\bar{R}_{5}=0$ (cf (67)). Note that $\bar{R}_{4} \neq \bar{R}_{5}$, and since $16 \operatorname{det}(\Omega)=-\left(\bar{R}_{4}-\bar{R}_{5}\right)^{4}$ by $(58)$ we have $\rho(\Omega)=4$.

$\rho(M)=2$. By setting $\sigma_{3}^{M}=\operatorname{det}(M)=0$ to zero we find all cases with $\rho(M)<3$, where we just have to exclude (84) and its permutations to get $\rho(M)=2$ (this is implicitly understood in the following). Generically, $M$ has a single zero eigenvalue. This comprises the case where all $R_{i}$ s coincide; by (81) it then follows that all of the $R_{i}$ s are zero, which is the case (87) below. Otherwise it is always possible to solve $\sigma_{3}^{M}=0$ for one $\bar{w}_{i}^{2}$, and we may assume $\bar{R}_{3} \neq \bar{R}_{5}$. The zero eigenvalue becomes at least double iff $\sigma_{3}^{M}=\sigma_{2}^{M}=0$, which is the case iff

$\bar{w}_{3}^{2}=\frac{-\bar{R}_{5}^{2}\left(\bar{R}_{3}+\bar{R}_{4}\right)+\bar{w}_{4}^{2}\left(\bar{R}_{4}-\bar{R}_{5}\right)}{\bar{R}_{5}-\bar{R}_{3}}, \quad \bar{w}_{5}^{2}=\frac{-\bar{R}_{3}^{2}\left(\bar{R}_{4}+\bar{R}_{5}\right)+\bar{w}_{4}^{2}\left(\bar{R}_{4}-\bar{R}_{3}\right)}{\bar{R}_{3}-\bar{R}_{5}}$

(note that different subcases have already been discussed in the tables). There is a triple zero eigenvalue iff, in addition, $\sigma_{1}^{M}=0$, which is the case (65) of a nilpotent $\mathrm{M}$ of index 3; the last three rows of table 6 produce examples fitting in the subsequent discussion of $\rho(\Omega)$.

Generically, we have $\rho(\Omega)=4$. For $\rho(\Omega)<4$ we have two possibilities. If (83) holds then $\operatorname{det}(\Omega)=0$ automatically, and $\operatorname{det}(M)=0 \Leftrightarrow \bar{R}_{5}\left(\bar{w}_{4}^{2}+\bar{w}_{5}^{2}\right)=0$, which leads to (87) and a subcase of (88). If (83) or its permutations do not hold, we necessarily have (e.g., $\left(\bar{R}_{4}-\bar{R}_{5}\right)\left(\bar{R}_{3}-\bar{R}_{4}-\bar{R}_{5}\right) \neq 0$ ) and the conditions $\operatorname{det}(M)=0=\operatorname{det}(\Omega)$ are solved simultaneously by

$$
\begin{aligned}
& \bar{w}_{4}^{2}=\frac{\left(\bar{R}_{3}-\bar{R}_{4}+\bar{R}_{5}\right)^{2}}{\bar{R}_{4}-\bar{R}_{5}}\left[\frac{\bar{R}_{5}-\bar{R}_{3}}{\left(\bar{R}_{3}-\bar{R}_{4}-\bar{R}_{5}\right)^{2}} \bar{w}_{3}^{2}+\frac{1}{4} \bar{R}_{5}\right], \\
& \bar{w}_{5}^{2}=\frac{\left(\bar{R}_{3}+\bar{R}_{4}-\bar{R}_{5}\right)^{2}}{\bar{R}_{4}-\bar{R}_{5}}\left[\frac{\bar{R}_{3}-\bar{R}_{4}}{\left(\bar{R}_{3}-\bar{R}_{4}-\bar{R}_{5}\right)^{2}} \bar{w}_{3}^{2}-\frac{1}{4} \bar{R}_{4}\right] .
\end{aligned}
$$

This generically corresponds to $\rho(\Omega)=3$.

The case $\rho(\Omega)=2$ arises for two different choices of the parameters.

(1) The first possibility is

$$
\bar{R}_{i}=0, \quad i=3,4,5
$$

This is precisely spin type $\{(000)\}_{\|}[\bar{R}=0]$. In this case the extended Segre types are $M[ \pm \mathrm{i} \omega(Z \bar{Z}), 0], \Omega[ \pm \mathrm{i} \omega(Z \bar{Z}), 0(11)]$ and thus $\mathrm{C}[\mathrm{i} \omega(Z 111),-\mathrm{i} \omega(\bar{Z} 111), 0(1111)]$.

(2) The second possibility is

$$
\bar{R}_{5}=\bar{R}_{4}-\bar{R}_{3}, \quad \bar{w}_{4}=0, \quad \bar{w}_{5}^{2}=\frac{\bar{R}_{3}\left(\bar{R}_{3} \bar{R}_{4}-\bar{R}_{4}^{2}-\bar{w}_{3}^{2}\right)}{\bar{R}_{4}-\bar{R}_{3}},
$$

which can be understood as a subcase of (86). The further subcase hereof

$$
\bar{R}_{4}=0, \quad \bar{w}_{3}^{2}=\frac{1}{2} \bar{R}_{3}^{2},
$$

is precisely the case (59) of a nilpotent C (cf also table 6).

A summary for the possible relative ranks of $M$ and $\Omega$ is given in table 8 .

6.2.5. $\operatorname{Ker}(\mathrm{M})$ and $\operatorname{Ker}(\Omega)$. From (49) it is easy to see that $\operatorname{Ker}(\mathrm{M}) \preceq \operatorname{Ker}(\mathrm{C})$. Of course, in the generic case $\mathrm{M}$ and $\Omega$ have full rank (i.e. $\rho(\mathrm{M})=3$ and $\rho(\Omega)=4$ ), so that $\operatorname{Ker}(\mathrm{M})=\{0\}$ and $\operatorname{Ker}(\Omega)=\{0\}$. The spin type is generically $\{111\}_{g}[\bar{R} \neq 0]$, but many special subcases are possible. In particular, we have the type $\{(000)\}_{\|}[\bar{R} \neq 0]$ if $\bar{R}_{3}=\bar{R}_{4}=\bar{R}_{5}$, or $\{(000)\}_{0}[\bar{R} \neq 0]$ if, additionally, $\bar{w}=0$. For these two simple types the conditions $\rho(\mathrm{M})=3$ and $\rho(\Omega)=4$ are, in fact, necessary.

The more special cases of table 8 are now discussed. here.

$\rho(M)=3, \rho(\Omega)=3$. This case is defined by either (82) or (83). Clearly $\operatorname{Ker}(M)=\{0\}$ 
Table 8. Weyl operator geometry for type II spacetimes: possible relative ranks of $M$ and $\Omega$ and equation numbers of the corresponding conditions (up to permutations of the axes).

\begin{tabular}{lllll}
\hline & $\rho(\Omega)=4$ & $\rho(\Omega)=3$ & $\rho(\Omega)=2$ & $\rho(\Omega)=1$ \\
\hline$\rho(M)=3 \Leftrightarrow(81)$ & Generic case & $(82)$ or $(83)$ & - & - \\
$\rho(M)=2$ & $(92)$ & $(86)$ & $(87)$ or $(88)$ & - \\
$\rho(M)=1$ & $(84)$ & - & - & - \\
\hline
\end{tabular}

(1) When (82) is satisfied we find that

$$
\begin{aligned}
\operatorname{Ker}(\Omega)=\left\langle\mathbf{W}^{+}\right\rangle, \quad \mathbf{W}^{+}= & {\left[\left(\bar{R}_{4}-\bar{R}_{5}\right)^{2}-\bar{R}_{3}^{2}\right] \mathbf{W}+2\left(\bar{R}_{4}-\bar{R}_{5}+\bar{R}_{3}\right) \bar{w}_{4} \mathbf{W}_{[53]} } \\
& +2\left(\bar{R}_{5}+\bar{R}_{3}-\bar{R}_{4}\right) \bar{w}_{5} \mathbf{W}_{[34]}+\sqrt{\frac{\mathcal{P}\left[\bar{R}_{3}^{2}-\left(\bar{R}_{4}-\bar{R}_{5}\right)^{2}\right]}{\bar{R}_{3}-\bar{R}_{4}-\bar{R}_{5}}} \mathbf{W}_{[45]} .
\end{aligned}
$$

The spin type is generically $\{111\}_{g}[\bar{R} \neq 0]$, but many special subcases are possible.

(2) If, instead, (83) holds we get

$$
\operatorname{Ker}(\Omega)=\left\langle\mathbf{W}^{*}\right\rangle, \quad \mathbf{W}^{*}=-\bar{w}_{5} \mathbf{W}_{[53]}+\bar{w}_{4} \mathbf{W}_{[34]} .
$$

Here the spin type is $\{(11) 1\}_{g}[\bar{R} \neq 0]$; it specializes to $\{(11) 1\}_{\perp}[\bar{R} \neq 0]$ if $\bar{w}_{3}=0$.

$\rho(M)=2, \rho(\Omega)=4$. It is easy to see that if $\bar{R}_{3}=\bar{R}_{4}=\bar{R}_{5}=0$ then $\rho(\Omega)=2$; therefore, here we can assume that the $\bar{R}_{i}$ are not all identically zero. Let us take, for definiteness, $\bar{R}_{3} \neq 0$. Then, from (81) the condition $\operatorname{det}(M)=0$ gives

$$
\bar{w}_{3}^{2}=-\frac{\bar{R}_{3} \bar{R}_{4} \bar{R}_{5}+\bar{R}_{4} \bar{w}_{4}^{2}+\bar{R}_{5} \bar{w}_{5}^{2}}{\bar{R}_{3}} .
$$

It is now straightforward to see that

$$
\operatorname{Ker}(\mathrm{M})=\left\langle\mathbf{V}^{+}\right\rangle, \quad \mathbf{V}^{+}=\frac{\bar{R}_{4} \bar{w}_{4}^{2}+\bar{R}_{5} \bar{w}_{5}^{2}}{\bar{R}_{3}} \mathbf{V}_{3}-\left(\bar{R}_{5} \bar{w}_{5}+\bar{w}_{3} \bar{w}_{4}\right) \mathbf{V}_{4}+\left(\bar{R}_{4} \bar{w}_{4}-\bar{w}_{3} \bar{w}_{5}\right) \mathbf{V}_{5},
$$

where we substitute $\bar{w}_{3}$ from (92). Again, the spin type is generically $\{111\}_{g}[\bar{R} \neq 0]$, but many special subcases are possible.

$\rho(M)=2, \rho(\Omega)=3$. Using (86) we can easily compute the generators of the onedimensional spaces $\operatorname{Ker}(\mathrm{M})$ and $\operatorname{Ker}(\Omega)$; the expressions are rather long and non-illuminating, and are therefore omitted.

The spin type is in general $\{111\}_{g}[\bar{R} \neq 0]$. This specializes to $\{(11) 1\}_{g}[\bar{R} \neq 0]$ (but in a non-canonical frame) if $\bar{R}_{4}=\bar{R}_{3}$, or to $\{111\}_{g}[\bar{R}=0]$ if $\bar{R}_{5}=-\bar{R}_{3}-\bar{R}_{4}$. When $\bar{w}_{3}=0$ we get the type $\{111\}_{\perp}[\bar{R} \neq 0]$, which becomes $\{111\}_{\perp}[\bar{R}=0]$ if, additionally, $\bar{R}_{5}=-\bar{R}_{3}-\bar{R}_{4}$. Further, the type is $\{110\}_{g}[\bar{R} \neq 0]$ if $\bar{R}_{5}=2 \bar{R}_{3}-\bar{R}_{4}$; this becomes $\{110\}_{\perp 0}[\bar{R} \neq 0]$ with the further condition $\bar{w}_{3}=0$, or $\{110\}_{\perp 1}[\bar{R} \neq 0]$ for $\bar{w}_{4}=0$.

$\rho(M)=2, \rho(\Omega)=2$. There are two different possibilities corresponding to this situation.

(1) When (87) holds we get

$$
\begin{aligned}
& \operatorname{Ker}(\mathbf{M})=\left\langle\mathbf{V}^{*}\right\rangle, \quad \mathbf{V}^{*}=\bar{w}_{3} \mathbf{V}_{3}+\bar{w}_{4} \mathbf{V}_{4}+\bar{w}_{5} \mathbf{V}_{5}, \\
& \operatorname{Ker}(\Omega)=\left\langle\mathbf{W}^{* 1}, \mathbf{W}^{* 2}\right\rangle, \quad \mathbf{W}^{* 1}=-\bar{w}_{5} \mathbf{W}_{[45]}+\bar{w}_{3} \mathbf{W}_{[34]}, \quad \mathbf{W}^{* 2}=-\bar{w}_{4} \mathbf{W}_{[45]}+\bar{w}_{3} \mathbf{W}_{[53]} .
\end{aligned}
$$

This corresponds univocally to spin type $\{(000)\}_{\|}[\bar{R}=0]$. 
Table 9. Possible values of $\rho(\mathbf{C})$ for all permitted combinations of values of $\rho(M)$ and $\rho(\Omega)$ in type II spacetimes.

\begin{tabular}{llll}
\hline & $\rho(\Omega)=4$ & $\rho(\Omega)=3$ & $\rho(\Omega)=2$ \\
\hline$\rho(M)=3$ & $\rho(\mathrm{C})=10$ & $\rho(\mathrm{C})=9$ & - \\
$\rho(M)=2$ & $8 \leqslant \rho(\mathrm{C}) \leqslant 9$ & $7 \leqslant \rho(\mathrm{C}) \leqslant 9$ & $6 \leqslant \rho(\mathrm{C}) \leqslant 8$ \\
$\rho(M)=1$ & $6 \leqslant \rho(\mathrm{C}) \leqslant 8$ & - & - \\
\hline
\end{tabular}

(2) Assuming, instead, that (88) is satisfied we obtain

$$
\begin{aligned}
& \operatorname{Ker}(\mathrm{M})=\left\langle\mathbf{V}^{\times}\right\rangle, \mathbf{V}^{\times}=\bar{w}_{5}\left(\bar{R}_{3}-\bar{R}_{4}\right) \mathbf{V}_{3}-\bar{R}_{3}\left(\bar{R}_{3}-\bar{R}_{4}\right) \mathbf{V}_{4}+\bar{w}_{3} \bar{R}_{3} \mathbf{V}_{5}, \\
& \operatorname{Ker}(\Omega)=\left\langle\mathbf{W}_{[53]}, \mathbf{W}^{\times}\right\rangle, \mathbf{W}^{\times}=\bar{w}_{5}\left(\bar{R}_{3}-\bar{R}_{4}\right) \mathbf{W}+\bar{w}_{3} \bar{w}_{5} \mathbf{W}_{[45]}+\left(\bar{R}_{3} \bar{R}_{4}-\bar{R}_{4}^{2}-\bar{w}_{3}^{2}\right) \mathbf{W}_{[34]},
\end{aligned}
$$

where it is understood that $\bar{w}_{5}$ is as in (88). We also recall the special subcase (89) where both $M$ and $\Omega$ are nilpotent.

The spin type is in general $\{111\}_{\perp}[\bar{R} \neq 0]$. It becomes $\{(11) 1\}_{\|}[\bar{R} \neq 0]$ when $\bar{R}_{3}=0$ (and $\{(11) 1\}_{0}[\bar{R} \neq 0]$ if, additionally, $\bar{w}_{3}=0$ ); this is the situation, for instance, for the Kerr black string (where $\bar{w}_{3}=0$ in the equatorial plane). In addition, we may have the type $\{110\}_{\perp 0}[\bar{R}=0]$ for $\bar{R}_{4}=0$ (and $\{110\}_{0}[\bar{R}=0]$ if also $\bar{w}_{3}=0$ ), which includes the case when $\mathrm{C}$ is nilpotent; or $\{111\}_{\|}[\bar{R} \neq 0]$ if, instead, $\bar{w}_{3}=0$.

$\rho(M)=1$. The conditions for this to occur were already given in (84) (and correspond to a specialization of (92) above). Now we have (without loss of generality we can assume $\left.\bar{R}_{4} \neq 0\right)$

$$
\begin{aligned}
& \operatorname{Ker}(\mathrm{M})=\left\langle\mathbf{V}_{3}, \mathbf{V}^{0}\right\rangle, \quad \mathbf{V}^{0}=-\frac{\bar{w}_{3}}{\bar{R}_{4}} \mathbf{V}_{4}+\mathbf{V}_{5}, \\
& \operatorname{Im}(\mathrm{M})=\left\langle-\bar{R}_{4} \mathbf{V}_{4}+\bar{w}_{3} \mathbf{V}_{5}\right\rangle,
\end{aligned}
$$

while $\operatorname{Ker}(\Omega)=\{0\}$. If $\bar{R} \neq 0$ then $\mathrm{M}$ also admits a non-zero eigenvalue $-\bar{R} / 2=$ $\left(\bar{w}_{3}^{2}-\bar{R}_{4}^{2}\right) /\left(2 \bar{R}_{4}\right)$, with eigendirection $\operatorname{Im}(\mathrm{M})$. If $\bar{R}=0$ then $\mathrm{M}$ is nilpotent and $\operatorname{Im}(\mathrm{M})$ coincides with the subspace $\left\langle\mathbf{V}^{0}\right\rangle$ of $\operatorname{Ker}(\mathrm{M})$.

Here the spin type is in general $\{111\}_{\|}[\bar{R} \neq 0]$. It specializes to $\{(11) 1\}_{0}[\bar{R} \neq 0]$ for $\bar{R}_{5}=0$, and to $\{110\}_{\| 0}[\bar{R}=0]$ for $\bar{R}_{5}=-\bar{R}_{4}$ (in which case $\mathrm{M}$ is nilpotent). The Segre types of $M, \Omega$ and $\mathrm{C}$ are easy to determine (some cases are presented below).

6.2.6. $\rho(\mathrm{C})$ in the various subcases. We have

$$
6 \leqslant 2 \rho(M)+\rho(\Omega) \leqslant \rho(C) \leqslant \min \{\rho(M)+7, \rho(\Omega)+6\} \leqslant 10 .
$$

All inequalities are a priori clear from (49), except for $6 \leqslant 2 \rho(M)+\rho(\Omega)$ which follows from the detailed discussion above. Considering the various previous subcases we thus obtain the possibilities summarized in table 9. In general, the precise value of $\rho(\mathbb{C})$ also depends on the negative b.w. components.

\subsection{Type $\boldsymbol{D}$ spacetimes}

Type II spacetimes specialize to type $\mathbf{D}$ when $\check{C}_{K}^{t}=\check{H}=\check{C}_{-K}=0$ in (49). In this case, $\mathcal{U}, \mathcal{W}$ and $\mathcal{V}$ are all invariant subspaces (recall also (20)-(22)) and the equality $\rho(\mathbf{C})=2 \rho(M)+\rho(\Omega)$ holds. Therefore, in each case $\rho(\mathrm{C})$ sticks to the lower value given in table 9 and we can explicitly present the Kernel and Image of $\mathrm{C}$, as follows. 


$$
\begin{aligned}
& \rho(M)=3, \rho(\Omega)=4 . \text { Here } \rho(\mathrm{C})=10, \text { so that } \\
& \operatorname{Ker}(\mathrm{C})=\{0\}, \quad \operatorname{Im}(\mathrm{C})=\mathcal{U} \oplus \mathcal{W} \oplus \mathcal{V} .
\end{aligned}
$$

Both the Schwarzschild-Tangherlini and Myers-Perry solutions belong to this class. $\rho(M)=3, \rho(\Omega)=3$. Here $\rho(C)=9$, and we have

$$
\operatorname{Ker}(\mathbf{C})=\left\langle\mathbf{W}^{+}\right\rangle, \quad \operatorname{Im}(\mathbf{C})=\mathcal{U} \oplus \operatorname{Im}\left(\left.\Omega\right|_{\mathcal{W} \backslash\left\langle\mathbf{W}^{+}\right\rangle}\right) \oplus \mathcal{V} .
$$

with $\mathbf{W}^{+}$defined as in (90).

$$
\rho(M)=2, \rho(\Omega)=4 \text {. Here } \rho(\mathrm{C})=8 \text { and }
$$

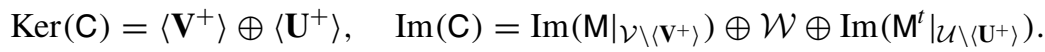

with $\mathbf{V}^{+}$as in (93) and $\mathbf{U}^{+}$defined analogously (i.e. $\operatorname{Ker}\left(\mathrm{M}^{t}\right)=\left\langle\mathbf{U}^{+}\right\rangle$).

$$
\rho(M)=2, \rho(\Omega)=3 \text {. Now } \rho(\mathrm{C})=7 \text { and }
$$

$$
\begin{aligned}
& \operatorname{Ker}(C)=\operatorname{Ker}(M) \oplus \operatorname{Ker}\left(M^{t}\right) \oplus \operatorname{Ker}(\Omega), \\
& \operatorname{Im}(C)=\operatorname{Im}\left(\left.M\right|_{\mathcal{V} \backslash \operatorname{Ker}(M)}\right) \oplus \operatorname{Im}\left(\left.\Omega\right|_{\mathcal{W} \backslash \operatorname{Ker}(\Omega)}\right) \oplus \operatorname{Im}\left(M^{t} \mid \mathcal{U} \backslash \operatorname{Ker}\left(\mathrm{M}^{t}\right),\right.
\end{aligned}
$$

with long expressions for $\operatorname{Ker}(\mathrm{M}), \operatorname{Ker}\left(\mathrm{M}^{t}\right)$ and $\operatorname{Ker}(\Omega)$, that we omit.

$$
\rho(M)=2, \rho(\Omega)=2 \text {. Now } \rho(\mathbf{C})=6 \text { and there are two possibilities, so that either }
$$

$$
\begin{aligned}
& \operatorname{Ker}(\mathrm{C})=\left\langle\mathbf{V}^{*}\right\rangle \oplus\left\langle\mathbf{U}^{*}\right\rangle \oplus\left\langle\mathbf{W}^{* 1}, \mathbf{W}^{* 2}\right\rangle, \\
& \operatorname{Im}(\mathrm{C})=\operatorname{Im}\left(\left.\mathrm{M}\right|_{\mathcal{V} \backslash\left\langle\mathbf{V}^{*}\right\rangle}\right) \oplus \operatorname{Im}\left(\left.\Omega\right|_{\mathcal{W} \backslash\left\langle\mathbf{W}^{* 1}, \mathbf{W}^{* 2}\right\rangle}\right) \oplus \operatorname{Im}\left(\mathbf{M}^{t} \mid \mathcal{U} \backslash\left\langle\mathbf{U}^{*}\right\rangle\right. \\
&
\end{aligned}
$$

with the definitions of (94) (and $\operatorname{Ker}\left(\mathrm{M}^{t}\right)=\left\langle\mathbf{U}^{*}\right\rangle$ ), or

$$
\begin{aligned}
& \operatorname{Ker}(\mathbf{C})=\left\langle\mathbf{V}^{\times}\right\rangle \oplus\left\langle\mathbf{U}^{\times}\right\rangle \oplus\left\langle\mathbf{W}_{[53]}, \mathbf{W}^{\times}\right\rangle, \\
& \operatorname{Im}(\mathbf{C})=\operatorname{Im}\left(\left.\mathbf{M}\right|_{\mathcal{V} \backslash\left\langle\mathbf{V}^{\times}\right\rangle}\right) \oplus \operatorname{Im}\left(\left.\Omega\right|_{\mathcal{W} \backslash\left\langle\mathbf{W}_{[33]}, \mathbf{W}^{\times}\right\rangle}\right) \oplus \operatorname{Im}\left(\left.\mathbf{M}^{t}\right|_{\mathcal{U} \backslash\left\langle\mathbf{U}^{\times}\right\rangle}\right),
\end{aligned}
$$

with the definitions of (95) (and $\left.\operatorname{Ker}\left(\mathrm{M}^{t}\right)=\left\langle\mathbf{U}^{\times}\right\rangle\right)$. For instance, the Kerr black string belongs to the latter class (see the text after (95)).

$$
\rho(M)=1, \rho(\Omega)=4 \text {. Also in this case } \rho(\mathrm{C})=6 \text {, but now }
$$

$\operatorname{Ker}(\mathbf{C})=\left\langle\mathbf{V}_{5}, \mathbf{V}^{0}\right\rangle \oplus\left\langle\mathbf{U}_{5}, \mathbf{U}^{0}\right\rangle, \quad \operatorname{Im}(\mathbf{C})=\operatorname{Im}\left(\left.\mathbf{M}\right|_{\mathcal{V} \backslash\left\langle\mathbf{V}_{5}, \mathbf{V}^{0}\right\rangle}\right) \oplus \mathcal{W} \oplus \operatorname{Im}\left(\left.\mathbf{M}^{t}\right|_{\mathcal{U} \backslash\left\langle\mathbf{U}_{5}, \mathbf{U}^{0}\right\rangle}\right)$,

with $\mathbf{V}^{0}$ as in (96) and $\mathbf{U}^{0}$ defined analogously (i.e. $\operatorname{Ker}\left(\mathrm{M}^{t}\right)=\left\langle\mathbf{U}_{5}, \mathbf{U}^{0}\right\rangle$ ). Cf (97) for the explicit form of $\operatorname{Im}(M)$.

\subsection{Comparison with $4 D$}

For $n=2$ we have $\bar{S}=0$ in addition to (10). Thus the only possible spin types for a 4D primary alignment type $\mathbf{I I}$ (i.e. Petrov type $\mathbf{I I}$ or $\mathbf{D}$ ) Weyl bivector operator are $\{(00)\}_{\|}[R \neq 0]$, $\{(00)\}_{\|}[R=0]$ and $\{(00)\}_{0}[R \neq 0]$. The first one is the generic case. In the type $\mathbf{D}$ subcase, in the second and third cases the Weyl tensor is dubbed purely magnetic and purely electric, respectively (see also section 7.2$)^{15}$.

The $2 \times 2$ matrices $M$ and $\Omega$ take the form [9]:

$$
M=\left[\begin{array}{cc}
-\frac{1}{4} \bar{R} & -\frac{1}{2} A_{34} \\
\frac{1}{2} A_{34} & -\frac{1}{4} \bar{R}
\end{array}\right], \quad \Omega=\left[\begin{array}{cc}
\frac{1}{2} \bar{R} & -A_{34} \\
A_{34} & \frac{1}{2} \bar{R}
\end{array}\right],
$$

\footnotetext{
${ }^{15}$ Observe that $\bar{R}$ and $A_{34}$ correspond, in the Newman-Penrose notation, to the real and imaginary part of $\Psi_{2}$, respectively.
} 
with respective eigenvalues $-\frac{1}{4}\left(\bar{R} \pm 2 \mathrm{i} A_{34}\right)$ and $\frac{1}{2}\left(\bar{R} \pm 2 \mathrm{i} A_{34}\right)$. Thus the eigenvalue degeneracies are

$$
\begin{array}{lcc}
\text { Spin type }\{(00)\}_{\|}: M[Z \bar{Z}], & \Omega[W \bar{W}], & \mathrm{C}[(Z Z)(\bar{Z} \bar{Z})(W \bar{W})] ; \\
\text { Spin type }\{(00)\}_{0}: M[(11)], & \Omega[(11)], & \mathrm{C}[(1111)(11)] .
\end{array}
$$

For Petrov type $\mathbf{D}$ this also gives the extended Segre types for C; for Petrov type II the extended Segre types are $C[Z[2] \bar{Z}[2], W \bar{W}]$ and $C[(22)(11)]$, respectively. Note that neither $M$ nor $\Omega$ can be nilpotent, basically due to the fact that they are the sum of an antisymmetric matrix and a multiple of the identity (instead of a general symmetric matrix).

\subsection{Discussion}

We have studied the (refined) algebraic classification of the 5D primary alignment type II Weyl operator C from the perspectives of Segre type (of $M$ or $\Omega$, and also of $\rho(M)$ and $\rho(\Omega)$ ) and spin type. In particular, we have described the Segre type classifications by treating particular cases, and then determined other properties such as the values of the ranks of $M$ and $\Omega$, the relation with $\rho(\mathrm{C})$, and the kernels and images of $M$ and $\Omega$, within each particular case.

The nilpotence of $\mathrm{C}$ is of particular interest. We found that the type II Weyl operator C is nilpotent if and only if both $\mathrm{M}$ and $\Omega$ are nilpotent (and the Segre types of $M$ and $\Omega$ are $M[0(3)]$ and $\Omega[0(31)]$, respectively), and the operator $C$ is generically nilpotent of index 9 (but lower indices can occur). In order to illustrate the classification of a type II operator $\mathrm{C}$ we determined the possible spin types and the extended Segre types of $\Omega$ in the case of a nilpotent $M$; a summary of the allowed spin types and Segre types of $M$ and $\Omega$, and their compatibility, was given in table 6 .

The classification of a type II operator C from the viewpoint of the extended Segre type of $\Omega$ was also illustrated in the case where $\Omega$ has a quadruple eigenvalue. Remarkably, we found that the spin type classification forms a pure refinement of the $\Omega$-Segre type classification and that the extended Segre type of $M$ is almost always $M[Z \bar{Z} 1]$. A summary of the allowed spin types and Segre types of $\Omega$ and $M$, and their compatibility, in the case of a quadruple $\Omega$-eigenvalue was given in table 7 .

We then focussed attention on the special type D subcase of type II spacetimes. In this case, the Kernel and Image of $\mathbf{C}$ were explicitly presented, and the possible values of $\rho(\mathbf{C})$ for all permitted combinations of values of $\rho(M)$ and $\rho(\Omega)$ in type II spacetimes were given (see table 9).

In appendix $\mathrm{C}$ we discuss the classification of a type II Weyl operator based on its spin type. For spin types $\{\cdot\}_{0},\{\cdot\}_{\|}$and $\{(11) 1\}_{\perp}$ we present the degeneracies in the eigenvalue spectra of $\mathrm{M}, \Omega$ and $\mathrm{C}$, and briefly comment on the more general spin types. As was the case for type III, a certain spin type gives rise to several possible eigenvalue degeneracies, opposed to the situation in $4 \mathrm{D}$.

Finally, let us point out that several other properties of type II/D Einstein spacetimes in higher (in particular, five) dimensions have been studied in [14, 28].

\section{Types I and G}

In general dimensions, a type I Weyl tensor is characterized by having a single WAND [ $\ell]$ and no multiple WANDs. With respect to any null frame $\left(\boldsymbol{\ell}, \boldsymbol{n}, \mathbf{m}_{i}\right)$ all components of b.w. greater than $\zeta=1$ vanish, whereas those of b.w. 1 are not all zero:

$$
\hat{H}=0, \quad(\hat{n}, \hat{v}) \neq(0,0)
$$


If this is the unique single WAND the type is $\mathbf{I}_{0}$, otherwise the type is $\mathbf{I}_{i}$. If there are no WANDs at all, the Weyl type is $\mathbf{G}$.

In $4 \mathrm{D}$ type $\mathbf{G}$ does not occur (i.e. WANDs always exist), and if the type is $\mathbf{I}$ then there are exactly four WANDs (such that the type is automatically $\mathbf{I}_{i}$; this is the so called algebraically general case in 4D). In higher than four dimensions, however, type $\mathbf{G}$ is generic [2] and type $\mathbf{I}$ is algebraically special with respect to type $\mathbf{G}$. However, in many applications the presence of a multiple WAND is important, and thus the distinction between types I/G and types II/III/N also appears to be significant.

We further note that type $\mathbf{I}(\mathrm{A})$ [9] (i.e. type I (a) of [1]) corresponds to the collection of the spin types $\mathbf{I}\{\cdot\}_{0}$ (i.e. $\hat{v}=0$ ), and type $\mathbf{I}(\mathrm{B})$ to $\mathrm{I}\{(000)\}_{\|}(\hat{n}=0)$. See, e.g., $[29,30]$ for examples of type $\mathbf{I} / \mathbf{G}$ (vacuum) spacetimes in five dimensions.

\subsection{The 'electric' and 'magnetic' parts of the Weyl operator}

For the type $\mathbf{I} / \mathbf{G}$ case there is another split which may be useful [25]. The split can be done for any of the types, but it is probably most useful for the type $\mathbf{I} / \mathbf{G}$ cases (and also possibly for type D). The split utilizes the existence of a Cartan involution of the general linear group.

Consider the full Lorentz group $G=O(1,4)$. Let $K \cong O(4)$ be a maximal compact subgroup of $O(1,4)$. Then there exists a unique Cartan involution $\theta$ of $G$ with the following properties [31]: (i) $\theta$ is invariant under the adjoint action of $K: A d_{K}(\theta)=\theta$. (ii) $O(1,4)$ is $\theta$-stable. (iii) $\theta$ is the following automorphism of the Lie algebra $\mathfrak{g l}(n, \mathbb{R}): X \mapsto-X^{*}$, where $*$ denotes the adjoint (which is equal to the transpose here since the coefficients are real).

If $\theta_{1}$ and $\theta_{2}$ are two such Cartan involutions of $G$, associated with maximal compact subgroups $K_{1}$ and $K_{2}$, then there exists a $g \in G$ such that $\theta_{1}=\operatorname{Int}(g) \theta_{2} \operatorname{Int}\left(g^{-1}\right)$, where $\operatorname{Int}(g)$ is the inner automorphism by $g$. By a slight abuse of notation, we will denote any representation of $\theta$ simply by $\theta$.

First, let us consider the case when $\theta: T_{p} M \mapsto T_{p} M$. The above-mentioned requirements enable us to choose a unit time-like vector $\mathbf{u}$ that is $K$-invariant, and let us then choose the $\theta$ which has $\mathbf{u}$ as an eigenvector. Therefore, in the orthonormal basis, $\left\{\mathbf{u}, \mathbf{m}_{i=2, \ldots, 5}\right\}$, we have the matrix representation:

$$
\theta=\left(\theta_{b}^{a}\right)=\operatorname{diag}(-1,1,1,1,1) .
$$

We note that $\theta^{2}=1$. This consequently picks out a special time-like direction and any other $\theta_{2}$ is related to a (different) time-like vector $\mathbf{u}_{2}$.

Through the tensor structure of bivector space, we can let $\theta$ act on bivector space; explicitly, $\theta: F^{a b} \mapsto \theta^{a}{ }_{c} \theta_{d}^{b} F^{c d}$. By choosing the $\theta$ adapted to the time-like vector $\mathbf{u}=(\boldsymbol{\ell}+\boldsymbol{n}) / \sqrt{2}$, we get the matrix representation of $\theta$ acting on bivector space in a $(3+4+3)$-block form relative to the basis (16)-(17):

$$
\theta=\left[\begin{array}{lll}
0 & 0 & 1 \\
0 & \eta & 0 \\
1 & 0 & 0
\end{array}\right], \quad \eta=\operatorname{diag}(-1,1,1,1) .
$$

This will then act on the Weyl operator $\mathbf{C}$ through conjugation $\theta \mathbf{C} \theta$.

Since $\theta^{2}=1$, the eigenvalues of $\theta$ are \pm 1 . We can thus project the operator $C$ along the eigenspaces of $\theta$ :

$$
\mathbf{C}=\mathbf{C}_{+}+\mathbf{C}_{-}, \quad \text { where } \quad \mathbf{C}_{+}=\frac{1}{2}(\mathbf{C}+\theta \mathbf{C} \theta), \quad \mathbf{C}_{-}=\frac{1}{2}(\mathbf{C}-\theta \mathbf{C} \theta)
$$


Using the Weyl operator (18) we can then compute the components of the matrix representation of $\mathrm{C}_{ \pm}$relative to (16)-(17):

$$
\begin{aligned}
& \mathcal{C}_{+}= {\left[\begin{array}{cccc}
\frac{1}{2}\left(M+M^{t}\right) & \frac{1}{2}(\hat{K}+\check{K}) & \frac{1}{2}(\hat{L}+\check{L}) & \frac{1}{2}(\hat{H}+\check{H}) \\
\frac{1}{2}\left(\hat{K}^{t}+\check{K}^{t}\right) & -\Phi & 0 & -\frac{1}{2}\left(\hat{K}^{t}+\check{K}^{t}\right) \\
\frac{1}{2}\left(\hat{L}^{t}+\check{L}^{t}\right) & 0 & \bar{H} & \frac{1}{2}\left(\hat{L}^{t}+\check{L}^{t}\right) \\
\frac{1}{2}(\hat{H}+\check{H}) & -\frac{1}{2}(\hat{K}+\check{K}) & \frac{1}{2}(\hat{L}+\check{L}) & \frac{1}{2}\left(M+M^{t}\right)
\end{array}\right] } \\
& \mathcal{C}_{-}=\left[\begin{array}{cccc}
\frac{1}{2}\left(M-M^{t}\right) & \frac{1}{2}(\hat{K}-\check{K}) & \frac{1}{2}(\hat{L}-\check{L}) & \frac{1}{2}\left(\hat{H}-\check{H}^{t}\right) \\
-\frac{1}{2}\left(\hat{K}^{t}-\check{K}^{t}\right) & 0 & -A^{t} & -\frac{1}{2}\left(\hat{K}^{t}-\check{K}^{t}\right) \\
-\frac{1}{2}\left(\hat{L}^{t}-\check{L}^{t}\right) & A & 0 & \frac{1}{2}\left(\hat{L}^{t}-\check{L}^{t}\right) \\
-\frac{1}{2}(\hat{H}-\check{H}) & \frac{1}{2}(\hat{K}-\check{K}) & -\frac{1}{2}(\hat{L}-\check{L}) & \frac{1}{2}\left(M^{t}-M\right)
\end{array}\right] .
\end{aligned}
$$

Therefore, we can see that the components $C_{ \pm}$are the symmetric and anti-symmetric parts of the Weyl operator with respect to the Euclidean metric on bivector space. In 4D these components are referred to as the electric and magnetic parts of the Weyl tensor. In [25] these were defined as the higher dimensional electric and magnetic part of the Weyl tensors; thus, henceforth we will refer to the component $\mathrm{C}_{+}$as the electric part of the Weyl operator (tensor), while $C_{-}$will be referred to as the magnetic part. Note that, as in $4 \mathrm{D}$, these parts depend on the choice of a time-like vector $\mathbf{u}$ (and their representation with respect to a different time-like vector $\mathbf{u}_{2}$ will change accordingly).

For type I/G Weyl tensors it is cumbersome to say anything general about their eigenvalue structure; however, for purely electric or purely magnetic Weyl operators, we have the following [25]:

Theorem 7.1. A purely electric $\left(P E, \mathrm{C}_{-}=0\right)$ Weyl operator has only real eigenvalues. A purely magnetic $\left(P M, \mathrm{C}_{+}=0\right)$ Weyl operator has at least 2 zero eigenvalues while the remaining eigenvalues are purely imaginary.

This can be seen more easily if we switch to an orthonormal frame (see [9]). Then using a $(4+6)$-block form:

$\mathcal{C}_{+}=\left[\begin{array}{cc}S_{1} & 0 \\ 0 & S_{2}\end{array}\right], \quad \mathcal{C}_{-}=\left[\begin{array}{cc}0 & T \\ -T^{t} & 0\end{array}\right], \quad S_{1}, S_{2}$ symmetric, $T$ a $4 \times 6$ matrix.

In the purely electric case, the eigenvalues are the eigenvalues of $S_{1}$ and $S_{2}$, which are clearly real. In the purely magnetic case, we note that the matrix $T$ can be decomposed (using the singular value decomposition) as $T=g_{1} D g_{2}$, where $g_{1}$ and $g_{2}$ are $S O(4)$ and $S O(6)$ matrices, respectively, and $D$ is a diagonal $4 \times 6$ matrix $D=\operatorname{diag}\left(\lambda_{1}, \lambda_{2}, \lambda_{3}, \lambda_{4}\right)$. Consequently, a purely magnetic Weyl operator has eigenvalues $\left\{0,0, \pm \mathrm{i} \lambda_{1}, \pm \mathrm{i} \lambda_{2}, \pm \mathrm{i} \lambda_{3}, \pm \mathrm{i} \lambda_{4}\right\}$.

From the vanishing of the diagonal in (113) we see, in particular, that the b.w. 0 part of a PE Weyl tensor has spin type $\{\cdots\}_{0}$. The fact that PE implies only real eigenvalues is illustrated, for example, by the classification of the eigenvalue structure of the 5D type D Weyl operator in appendix $\mathrm{C}$. On the other hand, the converse is not true-these are only necessary criteria, not sufficient; indeed, among (C.14)-(C.79) in appendix C.2, one can find cases where the Weyl operator has only real eigenvalues (i.e. only 1 and 0 occurring), which thus constitute counterexamples to the converse (since $\bar{w} \neq 0$ and hence not PE).

For a PM Weyl tensor the b.w. 0 part has spin type $\{(000)\}_{\|}[\bar{R}=0]$ (the diagonal of (112) vanishes) which does indeed give purely imaginary eigenvalues, as illustrated by (C.43) in appendix C. 2 in the type $\mathbf{D}$ case. Also for the PM case the converse is not true, as is illustrated by (C.51) in appendix C.2 which has purely imaginary eigenvalues but is not PM. 


\subsection{Exact purely electric/magnetic solutions}

In 4D there have been many studies attempting to classify purely electric or magnetic solutions with various sources. There is a wealth of 4D purely electric Weyl spacetimes; e.g., all static spacetimes (or, more generally, all those admitting a shear- and vorticity-free time-like vector field), all spacetimes with spherical, hyperbolical or planar symmetry, all Bianchi type I spacetimes, and the Schwarzschild, C and Gödel metrics. However, only a very limited number of 4D purely magnetic Weyl spacetimes are known to exist. It has even been conjectured that purely magnetic vacuum or dust spacetimes do not exist (and this has been proved under quite mild conditions, but not in general; see, e.g., [32-36]). For an extensive review of known 4D purely magnetic Weyl solutions see [37]; see also [38, 39] for two more recent contributions. All known purely magnetic metrics are algebraically general (Petrov type I), except for the locally rotationally symmetric metrics given in [40, 41], which are of Petrov type D.

There are also many examples of purely electric spacetimes in $5 \mathrm{D}$, including static spacetimes, spacetimes with an $\mathbb{R}^{4}$ spatial translational invariance, spherically symmetric spacetimes, and spacetimes with $S O(2) \times S O(2)$ isotropy or with spatial isotropy $H \supset$ $S O(2) \times S O(2)$ [9]. Remarkably, with our general definition of Weyl electric and magnetic parts and of PE/PM spacetimes, many of the generic examples mentioned above can be lifted to five or higher dimensions - see [25] for full details. It would be useful to classify all such solutions. In addition, the purely magnetic spacetimes are much harder to find. The only purely magnetic spacetimes known so far in higher than four dimensions are conformally related to a two-parameter family of Riemann purely magnetic spacetimes; these are all type $\mathbf{I}_{i}$, see [25]. A complete algebraic classification may be helpful in the search for new exact 5D solutions.

\section{Conclusions and discussion}

In this paper we have presented a refinement of the null alignment classification of the Weyl tensor of a 5D spacetime based on the notion of spin type of the components of highest boost weight and the Segre types of the Weyl operator acting on bivector space (and we have examined the intersection between the two (sub)classifications). We have presented a full treatment for types $\mathbf{N}$ and III, and illustrated the classification from different viewpoints (Segre type, rank, spin type) for types II and $\mathbf{D}$, paying particular attention to possible nilpotence, since this is a completely new feature of higher dimensions. We also briefly discussed alignment types I and $\mathbf{G}$.

In future work we shall develop the algebraic classification further. In particular, it is hoped that canonical forms can be determined explicitly in each algebraic subcase. The analysis may be used to study particular spacetimes of special interest in detail; for example, stationary (static) spacetimes and warped product spacetimes. In particular, we could attempt to classify and analyze all vacuum Einstein type III spacetimes in 5D. We also note that the algebraic techniques may be of use in other applications, since the analysis is independent of any field equations.

This work is timely because of the recent interest in the study of general relativity (GR) in higher dimensions and, in particular, in higher dimensional black holes [10], motivated, in part, by supergravity, string theory and the gauge-gravity correspondence. Indeed, even at the classical level gravity in higher dimensions exhibits a much richer structure than in 4D. For example, there is no unique black hole solution in higher dimensions. In fact, there now exist a number of different asymptotically flat, higher dimensional vacuum black hole solutions [10], including Myers-Perry black holes [42], black rings [43, 44], and various solutions with multiple horizons (e.g., [45, 46]). 
Since the algebraic classification of spacetimes has played such a crucial role in understanding exact solutions in $4 \mathrm{D}$, it is likely to play an important role also in higher dimensions. However, compared to $4 \mathrm{D}$, the algebraic types defined by the higher dimensional alignment classification are rather broad and it has proven more difficult to derive general results. Therefore, it is important to develop more refined algebraic classifications, and it is hoped that the work in this paper will prove useful in the search and analysis of exact higher dimensional (and, in particular, 5D) black hole solutions.

It would also be useful to obtain a more constructive way of accessing the invariant classification information in higher dimensions. For example, in [24] discriminants were used to study the necessary conditions for the Weyl curvature operator (and hence the higher dimensional Weyl tensor) to be of algebraic type II or $\mathbf{D}$ in terms of simple scalar polynomial curvature invariants. In particular, the Sorkin-Gross-Perry soliton, the supersymmetric black ring, the doubly spinning black ring and a number of other higher dimensional spacetimes were investigated using discriminant techniques ${ }^{16}$.

\section{Acknowledgments}

AC was supported, in part, by NSERC. MO has been supported by research plan RVO: 67985840 and research grant GAČR P203/10/0749. LW has been supported by an Yggdrasil mobility grant no 211109 to Stavanger University, a BOF research grant of Ghent University, and a FWO mobility grant no V4.356.10N to Utrecht University, where parts of this work were performed. MO and LW are grateful to the Department of Mathematics and Statistics, Dalhousie University, for hospitality during the initial stages of this work.

\section{Appendix A. Jordan normal structure and projectors}

Let $V$ be the complexification of a real vector space of finite dimension $m$, and let $\mathrm{T}$ be a linear operator (endomorphism) on $V$. For any $\lambda \in \mathbb{C}$, define

$$
\mathrm{T}_{\lambda} \equiv \mathrm{T}-\lambda \mathrm{id}_{V}
$$

with $\operatorname{id}_{V}$ the identity transformation on $V$, and

$$
W_{k}^{(\lambda)} \equiv \operatorname{Ker}\left(T_{\lambda}^{k}\right), \quad k \in \mathbb{N} .
$$

Note that $W_{0}^{(\lambda)}=\{0\}$. One can show that a smallest natural number $s=s(\lambda), 0 \leqslant s \leqslant m$, exists such that for all $k \in \mathbb{N}$ :

$$
W_{0}^{(\lambda)} \supsetneqq W_{1}^{(\lambda)} \supsetneqq \cdots \supsetneqq W_{s-1}^{(\lambda)} \supsetneqq W_{s}^{(\lambda)}=W_{s+k}^{(\lambda)} .
$$

The complex number $\lambda$ is an eigenvalue of $\mathrm{T}$ if and only if $s(\lambda) \geqslant 1$, i.e. it is a solution of the characteristic equation $\operatorname{det}\left(\mathcal{T}-x \mathbf{1}_{m}\right)=0$ of $\mathrm{T}$, where $\mathbf{1}_{m}$ is the unit matrix of dimension $m$ and $\mathcal{T}$ is the representation matrix of $T$ with respect to any basis of $V$. In this case, $E_{\lambda} \equiv W_{1}^{(\lambda)} \neq\{0\}$ and $M^{(\lambda)} \equiv W_{s}^{(\lambda)}$ are called the eigenspace, respectively, generalized eigenspace of T corresponding to $\lambda$. Since $\mathrm{T}_{\lambda}\left(W_{k+1}^{(\lambda)}\right) \preceq W_{k}^{(\lambda)}$, the number $s(\lambda)$ is the index of nilpotence of the restriction of $\mathrm{T}_{\lambda}$ to $M^{(\lambda)}$. If $\left\{\lambda_{1}, \ldots, \lambda_{r}\right\}$ is the set of different eigenvalues of $\mathrm{T}$, then

$$
V=\bigoplus_{A=1}^{r} M^{\left(\lambda_{A}\right)}
$$

${ }^{16}$ Indeed, it was found that the Sorkin-Gross-Perry soliton spacetimes and the 5D supersymmetric rotating black holes are of type I or $\mathbf{G}$, while the doubly spinning black ring can only be of type II or more special at the horizon [47] (in fact, it is more generally known that at Killing horizons the Weyl type must be II or more special, at least under some assumptions on the matter content $[14,48])$. 
Let $\lambda$ be an eigenvalue of $\mathrm{T}$, take an arbitrary complementary subspace $W_{s}^{\prime(\lambda)}$ of $W_{s-1}^{(\lambda)}$ in $M^{(\lambda)}=W_{s}^{(\lambda)}$ :

$$
W_{s}^{(\lambda)}=W_{s-1}^{(\lambda)} \oplus W_{s}^{\prime \prime(\lambda)}
$$

and put $W_{s}^{\prime \prime(\lambda)}=W_{s}^{\prime(\lambda)}$ (for consistency with the next pair of equations). By induction, define for $k=1, \ldots, s-1$ an arbitrary complementary subspace $W_{s-k}^{\prime(\lambda)}$ of $W_{s-k-1}^{(\lambda)} \oplus \mathrm{T}_{\lambda}\left(W_{s-k+1}^{\prime \prime(\lambda)}\right)$ in $W_{s-k}^{(\lambda)}$ via

$$
\begin{aligned}
& W_{s-k}^{(\lambda)}=W_{s-k-1}^{(\lambda)} \oplus \mathrm{T}_{\lambda}\left(W_{s-k+1}^{\prime \prime(\lambda)}\right) \oplus W_{s-k}^{\prime(\lambda)}, \\
& W_{s-k}^{\prime \prime(\lambda)} \equiv \mathrm{T}_{\lambda}\left(W_{s-k+1}^{\prime \prime(\lambda)}\right) \oplus W_{s-k}^{\prime(\lambda)} .
\end{aligned}
$$

By construction, the dimensions $p_{k}=p_{k}(\lambda)$ of $W_{k}^{\prime(\lambda)}$ are independent of the choice of $W_{k}^{\prime(\lambda)}$. Let $\left(\mathbf{e}_{i_{k}}^{\lambda, k}\right)_{i_{k}=1, \ldots, p_{k}}$ be an arbitrary basis of $W_{k}^{\prime(\lambda)}, k=1, \ldots, s$. A sequence $\mathbf{e}_{i_{k}}^{\lambda}[k]$ of the form

$$
\mathbf{e}_{i_{k}}^{\lambda}[k] \equiv\left(\mathbf{e}_{i_{k}}^{\lambda, k}, \mathbf{T}_{\lambda}\left(\mathbf{e}_{i_{k}}^{\lambda, k}\right), \ldots, \mathbf{T}_{\lambda}^{k-1}\left(\mathbf{e}_{i_{k}}^{\lambda, k}\right)\right)
$$

is called a Jordan normal sequence (JNS) of length $k$, corresponding to $\lambda$. It follows that a basis of $M^{(\lambda)}$ is given by

$$
\bigcup_{k=1}^{s} \bigcup_{i_{k}=1}^{p_{k}} \mathbf{e}_{i_{k}}^{\lambda}[k]
$$

Note that, since $\mathbf{e}_{i_{k}}^{\lambda, k} \in W_{k}^{(\lambda)}=\operatorname{Ker}\left(\mathrm{T}_{\lambda}^{k}\right)$, the last element of a JNS, and only this one, is an element of $\operatorname{Ker}\left(\mathrm{T}_{\lambda}\right)=E_{\lambda}$, i.e. it is an eigenvector of $\mathrm{T}$ corresponding to $\lambda$. Combining (A.4)-(A.6) yields a basis of $V$, which is called a Jordan normal basis (JNB) for T. The matrix representation of $\mathrm{T}$ with respect to the JNB has a block-diagonal form, called the Jordan normal form of T; for a given eigenvalue $\lambda$ the corresponding JNSs of length $k$ give rise to $p_{k}(\lambda)$ Jordan blocks of dimension $k(1 \leqslant k \leqslant s(\lambda))$ :

$$
B_{\lambda}[k] \equiv\left[\begin{array}{ccccc}
\lambda & 0 & 0 & \cdots & 0 \\
1 & \lambda & 0 & \ddots & \vdots \\
0 & 1 & \lambda & \ddots & 0 \\
\vdots & \ddots & \ddots & \ddots & 0 \\
0 & \ldots & 0 & 1 & \lambda
\end{array}\right] .
$$

It follows that the algebraic multiplicity of the eigenvalue $\lambda$ (i.e. the power of $x-\lambda$ in the characteristic polynomial), $\operatorname{dim}\left(M^{(\lambda)}\right)$, and the geometric multiplicity, $\operatorname{dim}\left(E_{\lambda}\right)$, are given by

$$
\operatorname{dim}\left(M^{(\lambda)}\right)=\sum_{k=1}^{s(\lambda)} k p_{k}(\lambda), \quad \operatorname{dim}\left(E_{\lambda}\right)=\sum_{k=1}^{s(\lambda)} p_{k}(\lambda),
$$

the latter being the total number of Jordan blocks corresponding to the eigenvalue $\lambda$. In particular, $\operatorname{dim}(\operatorname{Ker}(\mathrm{T}))$ equals the total number $\sum_{k=1}^{s} p_{k}(0)=\operatorname{dim}\left(E_{0}\right)$ of blocks corresponding to $\lambda=0$. Also, blocks of dimension 1 give rise to a diagonal block of $\mathrm{T}$.

Consider a particular element $\lambda_{A}$ of the set $\left\{\lambda_{B}\right\}$ of eigenvalues of T. As $s\left(\lambda_{A}\right)$ is the index of nilpotence of $\mathrm{T}_{\lambda_{A}}$, it follows that $\perp_{A}$ defined by

$$
\perp_{A} \equiv \mathrm{id}_{V}-\left(\operatorname{id}_{V}-\tilde{\perp}_{A}\right)^{s\left(\lambda_{A}\right)}, \quad \tilde{\perp}_{A} \equiv \frac{\prod_{B \neq A} \mathrm{~T}_{\lambda_{B}}^{s\left(\lambda_{B}\right)}}{\prod_{B \neq A}\left(\lambda_{A}-\lambda_{B}\right)^{s\left(\lambda_{B}\right)}}
$$

is the projection operator on $M^{\left(\lambda_{A}\right)}$. Clearly, $\mathrm{id}_{V}=\sum_{A=1}^{r} \perp_{A}$, and one can use these projectors to decompose the operator $\mathrm{T}$ :

$$
\mathrm{T}=\mathrm{N}_{\mathrm{T}}+\sum_{A=1}^{r} \lambda_{A} \perp_{A} .
$$


This defines the nilpotent operator $\mathrm{N}_{\mathrm{T}}$ which contains all the information not encapsulated in the eigenvalues $\lambda_{A}$. The operator

$$
\mathrm{N}_{A} \equiv \perp_{A} \mathrm{~N}_{\mathrm{T}}=\mathrm{N}_{\mathrm{T}} \perp_{A}
$$

has index of nilpotence $s\left(\lambda_{A}\right)$, and since $\perp_{A} \perp_{B}=\delta_{A B} \perp_{A}$ and

$$
\mathrm{N}_{\mathrm{T}}=\sum_{A=1}^{r} \mathrm{~N}_{A}
$$

it follows that $\mathrm{N}_{\mathrm{T}}$ has index of nilpotence $\max _{A} s\left(\lambda_{A}\right)$. Combining (A.10) and (A.12), we get the orthogonal decomposition:

$$
\mathrm{T}=\sum_{A=1}^{r} \mathrm{~T}_{A}, \quad \mathrm{~T}_{A} \equiv \mathrm{N}_{A}+\lambda_{A} \perp_{A} .
$$

Note that for all $A, \perp_{A}$ and $\mathrm{N}_{A}$ are elements of the commutative algebra $\mathbb{Q}\left(\lambda_{1}, \ldots, \lambda_{r}\right)[\mathrm{T}]$, where $\mathbb{Q}\left(\lambda_{1}, \ldots, \lambda_{r}\right)$ is the field of rational functions in the eigenvalues $\lambda_{A}$ over $\mathbb{Q}$. Since the polynomial invariant $\operatorname{tr}\left(T^{l}\right)$ of $\mathrm{T}$ equals the sum of the $l$ th powers of the eigenvalues, namely $\sum_{A=1}^{r} \operatorname{dim}\left(M^{(\lambda)}\right) \lambda_{A}^{l}$, and since the coefficients of the characteristic equation of $\mathrm{T}$ are combinations of the latter via Newton's identities, the eigenvalues are uniquely determined by the polynomial invariants. It follows that $\perp_{A}$ and $\mathrm{N}_{A}$ can be determined (in principle) by taking powers of $\mathrm{T}$ and traces thereof.

Finally, given the eigenvalues of $\mathrm{T}$ its Jordan normal form can be easily determined by calculating $\rho\left(\mathrm{N}_{A}^{j}\right), j=0, \ldots, s\left(\lambda_{A}\right)-1$, with $\rho\left(\mathrm{N}_{A}^{0}\right) \equiv \operatorname{dim}\left(M^{\left(\lambda_{A}\right)}\right)$. Indeed, for $1 \leqslant i \leqslant k$ we have $\rho\left(\left[B_{\lambda_{A}}[k]-\lambda_{A} \mathbf{1}_{k}\right]^{j}\right)=k-j$. Hence,

$$
\rho\left(\mathrm{N}_{A}^{j}\right)=\sum_{k=j+1}^{s\left(\lambda_{A}\right)}(k-j) p_{k}\left(\lambda_{A}\right), \quad j=0, \ldots, s\left(\lambda_{A}\right)-1 .
$$

This gives a linear system of $s\left(\lambda_{A}\right)$ equations in the same number of unknowns $p_{k}\left(\lambda_{A}\right),{ }^{17}$ which can be readily solved to give

$$
p_{k}\left(\lambda_{A}\right)=\rho\left(\mathrm{N}_{A}^{k-1}\right)-2 \rho\left(\mathrm{N}_{A}^{k}\right)+\rho\left(\mathrm{N}_{A}^{k+1}\right), \quad k=1, \ldots, s\left(\lambda_{A}\right) .
$$

Given the eigenvalues, $N_{A}$ can be represented with respect to any basis, and the ranks of its powers can be easily determined by computing determinants.

\section{Appendix B. Details of the type III Weyl operator classification}

\section{B.1. Classification based on $\rho(\mathrm{C})$}

Let $(i j k)$ be a cyclic permutation of (345). We symbolize the order 2 minors $\check{C}_{K}\left(\begin{array}{ll}i_{1} & i_{2} \\ j_{1} & j_{2}\end{array}\right)$ of $\check{C}_{K}$ - up to sign — by (no summation over repeated indices)

$$
\begin{aligned}
& \alpha_{i} \equiv 2\left(\check{v}_{j}^{2}+\breve{v}_{k}^{2}\right), \quad \beta_{i} \equiv \check{v}_{i}^{2}+\check{n}_{j} \check{n}_{k}, \\
& \gamma_{i+}=\check{v}_{j} \check{v}_{k}+\check{n}_{i} \check{v}_{i}, \quad \gamma_{i-}=\check{v}_{j} \check{v}_{k}-\check{n}_{i} \check{v}_{i}, \\
& \delta_{i+}=2\left(\check{v}_{j} \check{v}_{k}+\check{n}_{j} \check{v}_{i}\right), \quad \delta_{i-}=2\left(\check{v}_{j} \check{v}_{k}-\check{n}_{k} \check{v}_{i}\right) .
\end{aligned}
$$

Adding to $\check{C}_{-K}$ its $i$ th row and taking the determinant we arrive at the identity

$$
2 \check{v}_{i} d+\check{n}_{i} d_{i}-\check{v}_{k} d_{j}+\check{v}_{j} d_{k}=0 \text {. }
$$

${ }^{17}$ Note that (A.14), $j=0$ is the first relation in (A.8), while subtracting (A.14), $j=1$ from it gives the second relation. 
Referring to (20)-(22) we have

$\rho(\mathrm{C})=3+\rho\left(\check{C}_{K}\right) \quad \Leftrightarrow \quad \rho(\mathrm{C})=6$ or $5 \quad \Leftrightarrow \quad \operatorname{Im}(\mathrm{C})=\operatorname{Im}\left(\left.\mathrm{C}\right|_{\mathcal{U}}\right) \oplus \operatorname{Im}\left(\check{\mathrm{C}}_{-\mathrm{K}}\right)$.

When $\rho(\mathrm{C})=4\left(\Rightarrow \rho\left(\check{C}_{K}\right)=2\right)$ the last sum is no longer direct (see also (B.12) below). This leads to the following discussion (see (38)-(43) for definitions).

- $\rho(\mathrm{C})=6 \Leftrightarrow \rho\left(C_{K}\right)=3 \Leftrightarrow D^{6} \neq 0$. In this case $\check{\mathrm{C}}_{\mathrm{K}}{ }^{t}$, and hence $\left.\mathrm{C}\right|_{\mathcal{U}}$, is one-to-one while $\check{C}_{-\mathrm{K}}$ maps onto $\mathcal{V}$. Hence, by (B.5):

$$
\begin{aligned}
& \operatorname{Im}(\mathrm{C})=\left\langle\mathrm{C}\left(\mathbf{U}_{3}\right), \mathrm{C}\left(\mathbf{U}_{4}\right), \mathrm{C}\left(\mathbf{U}_{5}\right)\right\rangle \oplus \mathcal{V}=\operatorname{Im}\left(\check{\mathrm{C}}_{\mathrm{K}}{ }^{t}\right) \oplus \mathcal{V}, \\
& \operatorname{Im}\left(\check{\mathrm{C}}_{\mathrm{K}}{ }^{t}\right)=\left\langle\check{\mathrm{C}}_{\mathrm{K}}{ }^{t}\left(\mathbf{U}_{3}\right), \check{\mathrm{C}}_{\mathrm{K}}{ }^{t}\left(\mathbf{U}_{4}\right), \check{\mathrm{C}}_{\mathrm{K}}{ }^{t}\left(\mathbf{U}_{5}\right)\right\rangle .
\end{aligned}
$$

As (B.4) is the $i$ th component of the vector relation $\check{C}_{-K} Y=0$, where

$$
Y \equiv\left[\begin{array}{llll}
d & d_{3} & d_{4} & d_{5}
\end{array}\right]^{t} \quad \leftrightarrow \quad \mathbf{Y} \equiv d \mathbf{W}+\sum_{i=3}^{5} d_{i} \mathbf{W}^{[j k]}
$$

we have that

$$
\operatorname{Ker}(\mathbf{C})=\operatorname{Ker}\left(\check{C}_{-\mathrm{K}}\right) \oplus \mathcal{V}, \quad \operatorname{Ker}\left(\check{C}_{-\mathrm{K}}\right)=\langle\mathbf{Y}\rangle
$$

- $\rho(\mathrm{C})<6 \Leftrightarrow \rho\left(C_{K}\right)=2 \Leftrightarrow D^{6}=0$. With the choice (41)-(42), for this case we have that

$$
\begin{aligned}
& \operatorname{Im}\left(\check{\mathrm{C}}_{\mathrm{K}}^{t}\right)=\left\langle\check{\mathrm{C}}_{\mathrm{K}}^{t}\left(\mathbf{U}_{3}\right), \check{\mathrm{C}}_{\mathrm{K}}^{t}\left(\mathbf{U}_{4}\right)\right\rangle, \quad \operatorname{Ker}\left(\check{\mathrm{C}}_{\mathrm{K}}{ }^{t}\right) \equiv\left\langle\mathbf{U}^{+}\right\rangle, \\
& \operatorname{Im}\left(\check{C}_{-K}\right)=\left\langle\left(\check{C}_{-K}\right)_{* j},\left(\check{C}_{-K}\right)_{* k}\right\rangle, \quad \operatorname{Ker}\left(\check{C}_{-\mathrm{K}}\right) \equiv\left\langle\mathbf{Y}^{1}, \mathbf{Y}^{2}\right\rangle .
\end{aligned}
$$

Here and in general, $N_{* i}\left(N_{i *}\right)$ stands for the $i$ th column (row) of a matrix $N$. One has $\mathbf{U}^{+} \in \mathcal{U}, \mathbf{U}^{+} \notin\left\langle\mathbf{U}_{3}, \mathbf{U}_{4}\right\rangle$ and $\mathrm{C}\left(\mathbf{U}^{+}\right) \in \mathcal{V}$, and

$$
\rho(\mathrm{C})=4 \quad \Leftrightarrow \quad D^{45}=0 \quad \Leftrightarrow \quad \mathrm{C}\left(\mathbf{U}^{+}\right) \in \operatorname{Im}\left(\check{C}_{-\mathrm{K}}\right) .
$$

In summary, we have that

(i) $\rho(\mathrm{C})=5 \Leftrightarrow D^{6}=0 \neq D^{45}$, where, with the aid of (B.5):

$$
\begin{aligned}
& \operatorname{Im}(\mathrm{C})=\left\langle\mathrm{C}\left(\mathbf{U}_{3}\right), \mathrm{C}\left(\mathbf{U}_{4}\right), \mathrm{C}\left(\mathbf{U}^{+}\right)\right\rangle \oplus \operatorname{Im}\left(\check{C}_{-\mathrm{K}}\right)=\operatorname{Im}\left(\check{\mathrm{C}}_{\mathrm{K}}{ }^{t}\right) \oplus \mathcal{V}, \\
& \operatorname{Ker}(\mathrm{C})=\operatorname{Ker}\left(\check{C}_{-\mathrm{K}}\right) \oplus \mathcal{V} .
\end{aligned}
$$

(ii) $\rho$ (C) $=4 \Leftrightarrow D^{6}=0=D^{45}$, where

$$
\begin{aligned}
& \operatorname{Im}(\mathbf{C})=\left\langle\mathbf{C}\left(\mathbf{U}_{3}\right), \mathbf{C}\left(\mathbf{U}_{4}\right)\right\rangle \oplus \operatorname{Im}\left(\check{C}_{-K}\right), \\
& \operatorname{Ker}(\mathbf{C})=\left\langle\mathbf{U}^{+}+\mathbf{W}^{+}\right\rangle \oplus \operatorname{Ker}\left(\check{C}_{-K}\right) \oplus \mathcal{V} .
\end{aligned}
$$

In appendix B.3 we will give explicit expressions for $\mathbf{Y}^{1}, \mathbf{Y}^{2}, \mathbf{W}^{+}$(which all belong to $\mathcal{W}), \mathbf{U}^{+}$and $D^{45}$, in the separate cases $\check{v}=0$ and $\check{v} \neq 0$.

\section{B.2. Subclassification based on $\rho\left(\mathrm{C}^{2}\right)$}

Referring to (36) we explicitly have

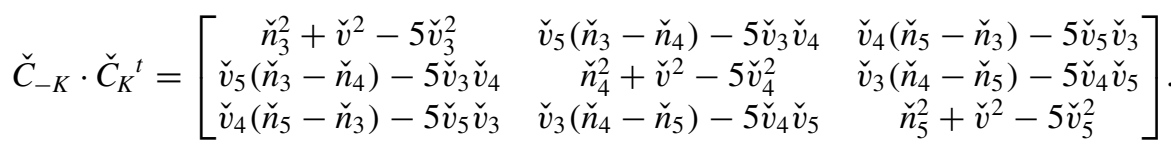


Referring to (20)-(22), the restriction of the map $\check{C}_{-K}$ to $\operatorname{Im}\left(\check{C}_{K}^{t}\right)$ induces the isomorphism of vector spaces

$$
\operatorname{Im}\left(C^{2}\right) \cong \frac{\operatorname{Im}\left(\check{C}_{\mathrm{K}}^{t}\right)}{\operatorname{Im}\left(\check{C}_{\mathrm{K}}^{t}\right) \cap \operatorname{Ker}\left(\check{C}_{-\mathrm{K}}\right)},
$$

such that

$$
\rho\left(\check{C}_{K}\right)-\rho\left(\mathrm{C}^{2}\right)=\operatorname{dim}\left(\operatorname{Im}\left(\check{\mathrm{C}}_{\mathrm{K}}^{t}\right) \cap \operatorname{Ker}\left(\check{\mathrm{C}}_{-\mathrm{K}}\right)\right) \text {. }
$$

For $\rho\left(\check{C}_{K}\right)=3$ we have $\operatorname{dim}\left(\operatorname{Im}\left(\check{C}_{\mathrm{K}}^{t}\right) \cap \operatorname{Ker}\left(\check{\mathrm{C}}_{-\mathrm{K}}\right)\right) \leqslant \operatorname{dim}\left(\operatorname{Ker}\left(\check{\mathrm{C}}_{-\mathrm{K}}\right)\right)=1$; regarding $\rho\left(\check{C}_{K}\right)=2$, and in view of (B.14), proposition 5.1 essentially states that the twodimensional vector spaces $\operatorname{Ker}\left(\check{C}_{-K}\right)$ and $\operatorname{Im}\left(\check{C}_{K}{ }^{t}\right)$ do not coincide. It follows that the difference $\rho\left(\check{C}_{K}\right)-\rho\left(\mathrm{C}^{2}\right)$ in (B.15) is either 0 or 1 , the latter case characterized by the existence of a (unique) bivector direction $\left\langle\mathbf{U}^{0}\right\rangle, \mathbf{U}^{0} \in \mathcal{U}$, satisfying

$0 \neq \check{C}_{\mathrm{K}}{ }^{t}\left(\mathbf{U}^{0}\right) \in \operatorname{Ker}\left(\check{C}_{-K}\right), \quad$ i.e. $\quad \mathrm{C}\left(\mathbf{U}^{0}\right) \notin \mathcal{V}, \quad \mathrm{C}^{2}\left(\mathbf{U}^{0}\right)=\check{\mathrm{C}}_{-\mathrm{K}}\left(\check{\mathrm{C}}_{\mathrm{K}}{ }^{t}\left(\mathbf{U}^{0}\right)\right)=0$.

This reconfirms the possible Segre types mentioned in table 5. For fixed value of $\rho(\mathrm{C})$ the first Segre type, corresponding to the highest value of $\rho\left(C^{2}\right)$, is the generic case. This leads to the following discussion (using also the corresponding previous results of subsection appendix B.1).

- $\rho(\mathrm{C})=6 \Leftrightarrow \rho\left(\check{C}_{K}\right)=3$. In this case $\rho\left(\mathrm{C}^{2}\right)$ is either 3 or 2 , where (cf (B.8))

$$
\rho\left(\mathrm{C}^{2}\right)=2 \quad \Leftrightarrow \quad D^{6<}=0 \quad \Leftrightarrow \quad\left\langle\check{\mathrm{C}}_{\mathrm{K}}{ }^{t}\left(\mathbf{U}^{0}\right)\right\rangle=\langle\mathbf{Y}\rangle .
$$

Here the determinant $D^{6<}$ is given by

$$
\begin{aligned}
D^{6<} & \equiv \operatorname{det}\left(\check{C}_{-K} \cdot C_{K}^{t}\right)=\operatorname{det}\left(\left[\begin{array}{c}
\left(\check{C}_{-K}\right)_{1 *} \\
\left(\check{C}_{-K}\right)_{2 *} \\
\left(\check{C}_{-K}\right)_{3 *}
\end{array}\right] \cdot\left[\left(C_{K}^{t}\right)_{* 1}\left(C_{K}^{t}\right)_{* 2}\left(C_{K}^{t}\right)_{* 3}\right]\right) \\
& =d^{2}-d_{3}^{2}-d_{4}^{2}-d_{5}^{2} \\
& =\operatorname{det}\left(\left[Y\left(\check{C}_{K}^{t}\right)_{* 1}\left(\check{C}_{K}^{t}\right)_{* 2}\left(\check{C}_{K}^{t}\right)_{* 3}\right]\right),
\end{aligned}
$$

with $d, d_{i}$ and $Y$ as defined in (38), (39) and (B.7). For later use, we notice that

$$
\rho(\mathrm{C})=6, d=0 \Rightarrow D^{6<} \neq 0 .
$$

* In the case $\rho\left(\mathrm{C}^{2}\right)=2 \leftrightarrow$ Segre type [(3322)], we have $W_{2}^{(0)} \equiv \operatorname{Ker}\left(\mathrm{C}^{2}\right)=$ $\mathcal{W} \oplus \mathcal{V} \oplus\left\langle\mathbf{U}^{0}\right\rangle$. If we take $\mathbf{U}_{3}^{\prime}, \mathbf{U}_{4}^{\prime}$ such that $\mathcal{U}=\left\langle\mathbf{U}_{3}^{\prime}, \mathbf{U}_{4}^{\prime}, \mathbf{U}^{0}\right\rangle$ and $\mathbf{W}^{*} \in \mathcal{W} \backslash \operatorname{Im}\left(\check{C}_{\mathrm{K}}^{t}\right)$, a Jordan basis is given by

$$
J N B=\left(\mathbf{U}_{3}^{\prime}[3], \mathbf{U}_{4}^{\prime}[3], \mathbf{U}^{0}[2], \mathbf{W}^{*}[2]\right),
$$

corresponding to $W_{3}^{\prime(0)}=\left\langle\mathbf{U}_{3}^{\prime}, \mathbf{U}_{4}^{\prime}\right\rangle$ and $W_{2}^{\prime(0)}=\left\langle\mathbf{U}^{0}, \mathbf{W}^{*}\right\rangle \cdot{ }^{18}$

$*$ In the generic case $\rho\left(\mathrm{C}^{2}\right)=3 \leftrightarrow$ Segre type [(3331)], we have $W_{2}^{(0)} \equiv \operatorname{Ker}\left(\mathrm{C}^{2}\right)=$ $\mathcal{W} \oplus \mathcal{V}$ and

$$
J N B=\left(\mathbf{U}_{3}[3], \mathbf{U}_{4}[3], \mathbf{U}_{5}[3], \mathbf{Y}[1]\right),
$$

corresponding to $W_{3}^{\prime(0)}=\mathcal{U}$ and $W_{1}^{\prime(0)}=\langle\mathbf{Y}\rangle$.

- $\rho(\mathrm{C})<6 \Leftrightarrow \rho\left(\check{C}_{K}\right)=2$. We choose $\mathbf{V}^{*} \in \mathcal{V} \backslash \operatorname{Im}\left(\check{C}_{-\mathrm{K}}\right)$ and write

$$
\mathbf{X} \equiv \begin{cases}\mathbf{U}^{+}[2], & \rho(\mathbf{C})=5 \\ \left(\mathbf{U}^{+}+\mathbf{W}^{+}\right)[1], \mathbf{V}^{*}[1], & \rho(\mathbf{C})=4 .\end{cases}
$$

In this case $\rho\left(\mathrm{C}^{2}\right)$ is either 2 or 1 . With the choice (41)-(42) we have

$$
\rho\left(\mathrm{C}^{2}\right)=1 \quad \Leftrightarrow \quad D^{45<}=0 \quad \Leftrightarrow \quad \check{\mathrm{C}}_{\mathrm{K}}{ }^{t}\left(\mathbf{U}^{0}\right) \in\left\langle\mathbf{Y}^{1}, \mathbf{Y}^{2}\right\rangle,
$$

${ }^{18}$ We have used the result $\operatorname{Im}\left(\check{C}_{K}^{t}\right)=\left\langle\check{C}_{K}^{t}\left(\mathbf{U}_{3}^{\prime}\right), \check{C}_{K}^{t}\left(\mathbf{U}_{4}^{\prime}\right), \mathbf{Y}\right\rangle$, which follows from (B.6) and (B.17). 
where the determinant $D^{45<}$ is given by

$$
\begin{aligned}
D^{45<} & \equiv \operatorname{det}\left(\left[\begin{array}{c}
\left(\check{C}_{-K}\right)_{1 *} \\
\left(\check{C}_{-K}\right)_{2 *}
\end{array}\right] \cdot\left[\left(C_{K}^{t}\right)_{* 1}\left(C_{K}^{t}\right)_{* 2}\right]\right) \\
& =\beta_{5}^{2}+\gamma_{3+}^{2}+\gamma_{4-}^{2}-\alpha_{5}^{2}-\delta_{3+}^{2}-\delta_{4-}^{2} \\
& =\left(\check{n}_{3}^{2}+\check{v}^{2}-5 \check{v}_{3}^{2}\right)\left(\check{n}_{4}^{2}+\check{v}^{2}-5 \check{v}_{4}^{2}\right)-\left(\check{v}_{5}\left(\check{n}_{3}-\check{n}_{4}\right)-5 \check{v}_{3} \check{v}_{4}\right)^{2} \\
& \sim \operatorname{det}\left(\left[Y^{1} Y^{2}\left(C_{K}{ }^{t}\right)_{* 1}\left(C_{K}{ }^{t}\right)_{* 2}\right]\right) .
\end{aligned}
$$

Here definitions (B.1)-(B.3) and (B.10) have been used, as well as the correspondence $Y^{1} \leftrightarrow \mathbf{Y}^{1}$ and $Y^{2} \leftrightarrow \mathbf{Y}^{2}$ between column vectors in $\mathbb{R}^{4 \times 1}$ and elements of $\mathcal{W}$, as in (B.7).

$*$ In the case $\rho\left(\mathbf{C}^{2}\right)=1$ we have $W_{2}^{(0)} \equiv \operatorname{Ker}\left(\mathbf{C}^{2}\right)=\mathcal{W} \oplus \mathcal{V} \oplus\left\langle\mathbf{U}^{+}\right\rangle \oplus\left\langle\mathbf{U}^{0}\right\rangle$. If we take $\mathbf{U}_{3}^{\prime}$ such that $\mathcal{U}=\left\langle\mathbf{U}_{3}^{\prime}, \mathbf{U}^{0}, \mathbf{U}^{+}\right)$, and

$$
\begin{gathered}
\mathbf{Y}^{*} \in \operatorname{Ker}\left(\check{\mathrm{C}}_{-\mathrm{K}}\right) \backslash\left\langle\check{\mathrm{C}}_{\mathrm{K}}{ }^{t}\left(\mathbf{U}^{0}\right)\right\rangle, \quad \mathbf{W}^{*} \in \mathcal{W} \backslash\left(\operatorname{Im} \check{\mathrm{C}}_{\mathrm{K}}{ }^{t}+\operatorname{Ker}\left(\check{\mathrm{C}}_{-\mathrm{K}}\right)\right) \\
=\mathcal{W} \backslash\left(\left\langle\mathbf{Y}^{1}, \mathbf{Y}^{2}\right\rangle \oplus\left\langle\check{\mathrm{C}}_{\mathrm{K}}{ }^{t}\left(\mathbf{U}_{3}^{\prime}\right)\right\rangle\right),
\end{gathered}
$$

a Jordan basis is then given by

$$
J N B=\left(\mathbf{U}_{3}^{\prime}[3], \mathbf{U}^{0}[2], \mathbf{W}^{*}[2], \mathbf{X}, \mathbf{Y}^{*}[1]\right),
$$

corresponding for $\rho(\mathbf{C})=5$ to $W_{3}^{\prime(0)}=\left\langle\mathbf{U}_{3}^{\prime}\right\rangle, W_{2}^{\prime(0)}=\left\langle\mathbf{U}^{0}, \mathbf{W}^{*}, \mathbf{U}^{+}\right\rangle, W_{1}^{\prime(0)}=\left\langle\mathbf{Y}^{*}\right\rangle$ and for $\rho(\mathbf{C})=4$ to $W_{3}^{\prime(0)}=\left\langle\mathbf{U}_{3}^{\prime}\right\rangle, W_{2}^{\prime(0)}=\left\langle\mathbf{U}^{0}, \mathbf{W}^{*}\right\rangle, W_{1}^{\prime(0)}=\left\langle\mathbf{U}^{+}+\mathbf{W}^{+}, \mathbf{V}^{*}, \mathbf{Y}^{*}\right\rangle$.

* In the generic case $\rho\left(C^{2}\right)=2$ we have $W_{2}^{(0)} \equiv \operatorname{Ker}\left(C^{2}\right)=\mathcal{W} \oplus \mathcal{V} \oplus\left\langle\mathbf{U}^{+}\right\rangle$. A Jordan basis is

$$
J N B=\left(\mathbf{U}_{3}[3], \mathbf{U}_{4}[3], \mathbf{X}, \mathbf{Y}^{1}[1], \mathbf{Y}^{2}[1]\right),
$$

corresponding for $\rho(\mathbf{C})=5$ to $W_{3}^{\prime(0)}=\left\langle\mathbf{U}_{3}, \mathbf{U}_{4}\right\rangle, W_{2}^{\prime(0)}=\left\langle\mathbf{U}^{+}\right\rangle, W_{1}^{\prime(0)}=\left\langle\mathbf{Y}^{1}, \mathbf{Y}^{2}\right\rangle$ and for $\rho(\mathbf{C})=4$ to $W_{3}^{\prime(0)}=\left\langle\mathbf{U}_{3}, \mathbf{U}_{4}\right\rangle, W_{1}^{\prime(0)}=\left\langle\mathbf{U}^{+}+\mathbf{W}^{+}, \mathbf{V}^{*}, \mathbf{Y}^{1}, \mathbf{Y}^{2}\right\rangle$.

\section{B.3. Intersection of the two refinements}

In the subsequent analysis we will use the normal forms for $(X, x)=(\check{n}, \breve{v})$ of table 2, which is compatible with (42) when $\breve{v} \neq 0$. Referring to these forms, it is advantageous to distinguish between the cases $\check{v}=0, \check{v}_{3} \neq 0=\check{v}_{4}=\check{v}_{5}, \check{v}_{3} \check{v}_{5} \neq 0=\check{v}_{4}$ and $\check{v}_{3} \check{v}_{4} \check{v}_{5} \neq 0$. For $\check{v}=0$ we will work with the choice (41).

B.3.1. Case $\check{v}=0$ (spin types $\{\cdot\}_{0}$ ). This case is characterized by $\mathbf{W} \in \operatorname{Ker}\left(\check{C}_{-K}\right) \supsetneqq \operatorname{Ker}(\mathbf{C})$ or diagonal $\check{L}$, cf (35). Regarding $\operatorname{Im}\left(\check{C}_{K}{ }^{t}\right)$, note that we may replace $\check{C}_{K}{ }^{t}\left(\mathbf{U}_{3}\right)$ by $\mathbf{W}_{[45]}$ etc in (B.6) and (B.9); thus $\operatorname{Im}\left(\check{\mathrm{C}}_{\mathrm{K}}{ }^{t}\right)=\left\langle\check{n}_{3} \mathbf{W}_{[45]}, \check{n}_{4} \mathbf{W}_{[53]}, \check{n}_{5} \mathbf{W}_{[34]}\right\rangle$ in a unified form for all values of $\rho(\mathrm{C})$.

Regarding $\rho(\mathrm{C})$ all $d_{i}$ s vanish, such that by (40):

$$
D^{6}=d^{2}=\left(\check{n}_{3} \check{n}_{4} \check{n}_{5}\right)^{2}=\operatorname{det}(\check{L})^{2} .
$$

Thus, with the choice (41):

$$
\rho(C)=6 \quad \Leftrightarrow \quad \check{n}_{5} \neq 0 \quad \rightarrow \quad\langle\mathbf{Y}\rangle=\langle\mathbf{W}\rangle .
$$

This precisely covers the primary spin types $\{(11) 1\}$ and $\{111\}$, while $\rho(C)<6$ is equivalent to primary type $\{110\}$. In the latter case we get $\operatorname{Im}\left(\check{\mathrm{C}}_{-\mathrm{K}}\right)=\left\langle\mathbf{V}_{3}, \mathbf{V}_{4}\right\rangle$ and

$$
\left\langle\mathbf{Y}^{1}, \mathbf{Y}^{2}\right\rangle=\left\langle\mathbf{W}, \mathbf{W}_{[34]}\right\rangle, \quad \mathbf{U}^{+}=\mathbf{U}^{5}, \quad D^{45}=\beta_{5}^{2} \check{H}_{55} .
$$

Thus

$$
\rho(\mathrm{C})=5 \quad \Leftrightarrow \quad \check{n}_{5}=0 \neq \check{H}_{55} ;
$$




$$
\rho(\mathrm{C})=4 \quad \Leftrightarrow \quad \check{n}_{5}=0=\check{H}_{55} \quad \rightarrow \quad \mathbf{W}^{+}=0 .
$$

Regarding $\rho\left(\mathbf{C}^{2}\right)$ we obtain

$$
\rho\left(\mathrm{C}^{2}\right)=\rho\left(\operatorname{diag}\left(\check{n}_{3}^{2}, \check{n}_{4}^{2}, \breve{n}_{5}^{2}\right)\right)=\rho\left(\operatorname{diag}\left(\check{n}_{3}, \check{n}_{4}, \check{n}_{5}\right)\right)=\rho(\check{L})=\rho\left(\check{C}_{K}\right),
$$

such that for fixed value of $\rho(\mathrm{C})$ the Segre type is always the generic one. Referring to (B.15) this tells that $\operatorname{Ker}\left(\check{C}_{-K}\right)$ and $\operatorname{Im}\left(\check{C}_{K}^{t}\right)$ only have the zero vector in common (as is readily checked), and is also in accordance with

$$
D^{6<}=d^{2} \neq 0[\rho(\mathrm{C})=6], \quad D^{45<}=\beta_{5}^{2} \neq 0[\rho(\mathrm{C})<6] .
$$

B.3.2. Specifications for $\breve{v} \neq 0$. With the choice (42), and referring to (40) and identity (B.4), it is easily seen that

$$
\rho(\mathrm{C})<6 \quad \Leftrightarrow \quad d_{3}=d_{4}=0 \text { or } d_{3}=d_{5}=0,
$$

respectively. None of these conditions are automatically satisfied; i.e. the generic situation for all $\check{v} \neq 0$ spin types is $\rho(\mathrm{C})=6$. Regarding $\rho(\mathrm{C})<6$, the choice $j=1, k=4 k$ naturally leads to the explicit expressions

$$
\left\langle\mathbf{Y}^{1}, \mathbf{Y}^{2}\right\rangle=\left\langle\alpha_{5} \mathbf{W}_{[45]}+\delta_{4-} \mathbf{W}_{[34]}-\gamma_{3+} \mathbf{W}, \alpha_{5} \mathbf{W}_{[53]}+\delta_{3+} \mathbf{W}_{[34]}-\gamma_{4-} \mathbf{W}\right\rangle
$$

and

$$
\begin{aligned}
& \mathbf{U}^{+}=\delta_{4+} \mathbf{U}_{3}+ \delta_{3-} \mathbf{U}_{4}-\alpha_{5} \mathbf{U}_{5}, \\
& \mathbf{W}^{+}=\left(-\delta_{4+} \check{v}_{3} \check{H}_{33}-\delta_{3-} \check{v}_{4} \check{H}_{44}+2\left(\check{n}_{5}\left(\check{v}_{3}^{2}-\check{v}_{4}^{2}\right)-2 \check{v}_{3} \check{v}_{4} \check{v}_{5}\right) \check{H}_{34}\right) \frac{\mathbf{W}}{\alpha_{5}} \\
&+ 2\left(-\delta_{4+} \check{v}_{4} \check{H}_{33}+\delta_{3-} \check{v}_{3} \check{H}_{44}+2\left(\check{v}_{5}\left(\check{v}_{3}^{2}-\check{v}_{4}^{2}\right)+2 \check{v}_{3} \check{v}_{4} \check{n}_{5}\right) \check{H}_{34}\right) \frac{\mathbf{W}_{[34]}}{\alpha_{5}} \\
&+\left(\check{v}_{3} \mathbf{W}+2 \check{v}_{4} \mathbf{W}_{[34]}\right) \check{H}_{35}+\left(\check{v}_{4} \mathbf{W}-2 \check{v}_{3} \mathbf{W}_{[34]}\right) \check{H}_{45}, \\
&-D^{45}=\delta_{4+}^{2} \check{H}_{33}+\delta_{3-}^{2} \check{H}_{44}+\alpha_{5}^{2} \check{H}_{55}+2 \delta_{3-} \delta_{4+} \check{H}_{34}-2 \delta_{4+} \alpha_{5} \check{H}_{35}-2 \delta_{3-} \alpha_{5} \check{H}_{45} .
\end{aligned}
$$

In the cases where $\rho\left(\mathbf{C}^{2}\right)=\rho\left(C_{K}\right)-1$, the bivector $\mathbf{U}^{0}$ can be determined from $\check{\mathbf{C}}_{K}^{t}\left(\mathbf{U}^{0}\right)=\mathbf{Y}$ by Cramer's method (case $\rho(\mathrm{C})=6, D^{6<}=0$ ) or from $a_{3} \mathrm{C}^{2}\left(\mathbf{U}_{3}\right)+a_{4} \mathrm{C}^{2}\left(\mathbf{U}_{4}\right)=0$ (case $\left.\rho(\mathrm{C})<6, D^{45<}=0\right)$.

B.3.3. Case $\check{v}_{3} \neq 0=\check{v}_{4}=\check{v}_{5}$ (spin types $\{\cdot\}_{\|}$and $\left.\{(11) 1\}_{\perp}\right)$. Regarding $\rho(\mathrm{C})$ we have

$$
d_{4}=d_{5}=0, \quad d_{3}=-2 \check{v}_{3}\left(\check{v}_{3}^{2}+\check{n}_{4} \check{n}_{5}\right), \quad d=\check{n}_{3}\left(\check{v}_{3}^{2}+\check{n}_{4} \check{n}_{5}\right),
$$

such that (cf (B.29))

$$
\rho(\mathrm{C})<6 \quad \Leftrightarrow \quad \check{v}_{3}^{2}+\check{n}_{4} \check{n}_{5}=0 .
$$

This condition is only compatible with $\check{n}_{4} \check{n}_{5} \neq 0$ and $\check{n}_{4} \neq \check{n}_{5}$, and thus occurs in the following cases:

$$
\begin{aligned}
& \{110\}_{\| 0}, \quad \check{n}_{3}=0, \check{n}_{5}=-\check{n}_{4}= \pm \check{v}_{3} ; \\
& \{111\}_{\|}, \quad \check{v}_{3}^{2}+\check{n}_{4} \check{n}_{5}=0 ; \\
& \{(11) 1\}_{\perp}, \quad \check{n}_{5}=-2 \check{n}_{3}=-2 \check{n}_{4}, \quad \check{v}_{3}^{2}=2 \check{n}_{3}^{2} .
\end{aligned}
$$


Then (B.30) and (B.33) reduce to

$$
\begin{aligned}
& \left\langle\mathbf{Y}^{1}, \mathbf{Y}^{2}\right\rangle=\left\langle-\check{n}_{3} \mathbf{W}+2 \check{v}_{3} \mathbf{W}_{[45]}, \check{n}_{4} \mathbf{W}_{[34]}+\check{v}_{3} \mathbf{W}_{[53]}\right\rangle, \\
& D^{45}=4 \check{n}_{4} \check{n}_{5}^{2}\left(\check{n}_{5} \check{H}_{44}-\check{n}_{4} \check{H}_{55}+2 \check{v}_{3} \check{H}_{45}\right) .
\end{aligned}
$$

Thus, for the cases (B.36)-(B.38) we have $\rho(\mathrm{C})=4$ if and only if

$$
\check{n}_{5} \check{H}_{44}-\check{n}_{4} \check{H}_{55}+2 \check{v}_{3} \check{H}_{45}=0 \text {, }
$$

else $\rho(\mathrm{C})=5$ (cf also remark 5.2). In all cases different from (B.36)-(B.38) we have $\rho(\mathrm{C})=6$ and, from (B.7) with (B.34):

$$
\langle\mathbf{Y}\rangle=\left\langle\check{n}_{3} \mathbf{W}-2 \check{v}_{3} \mathbf{W}_{[45]}\right\rangle .
$$

Regarding $\rho\left(\mathbf{C}^{2}\right)$ the governing determinants reduce to

$$
D^{6<}=\left(\check{n}_{3}^{2}-4 \check{v}_{3}^{2}\right)\left(\check{v}_{3}^{2}+\check{n}_{4} \check{n}_{5}\right)^{2}, \quad D^{45<}=\left(\check{n}_{3}^{2}-4 \check{v}_{3}^{2}\right)\left(\check{n}_{4}^{2}+\check{v}_{3}^{2}\right),
$$

such that, also in view of (B.35):

$$
\rho\left(\mathrm{C}^{2}\right)=\rho\left(\check{C}_{K}\right)-1 \quad \Leftrightarrow \quad \check{n}_{3}= \pm 2 \check{v}_{3} .
$$

In conjunction with (B.13) it follows that $\mathbf{U}^{0}=\mathbf{U}_{3}$ in this case. If $\rho(\mathrm{C})=6$, (B.40) $\leftrightarrow \rho\left(\mathrm{C}^{2}\right)=2$ is possible for spin types $\{(11) 1\}_{\|},\{110\}_{\| 1},\{111\}_{\|}$and $\{(11) 1\}_{\perp}$, but not for $\{(000)\}_{\|}$and $\{110\}_{\| 0}$. Regarding the $\rho($ C $)<6$ cases (B.36)-(B.38) only (B.37) allows for the subcase (B.40) giving $\rho\left(\mathrm{C}^{2}\right)=1$, namely

$$
\check{v}_{3}= \pm \frac{\check{n}_{3}}{2}, \quad \check{n}_{4}=-\frac{1+\sqrt{2}}{2} \check{n}_{3}, \quad \check{n}_{5}=-\frac{1-\sqrt{2}}{2} \check{n}_{3} .
$$

B.3.4. Case $\check{v}_{3} \check{v}_{5} \neq 0=\check{v}_{4}$ (spin types $\{111 / 0\}_{\perp}$ and $\{(11) 1\}_{g}$ ). From (39) we have

$$
d_{4}=2 \check{v}_{3} \check{v}_{5}\left(\check{n}_{5}-\check{n}_{3}\right) \neq 0
$$

such that $\rho(C)=6$. It is readily checked that $D^{6<}=0$ (see (B.18)) admits solutions for both spin types, leading to subcases where $\rho\left(\mathrm{C}^{2}\right)=2$.

B.3.5. Case $\check{v}_{3} \check{v}_{4} \breve{v}_{5} \neq 0$ (spin type $\{111 / 0\}_{g}$ ). First we take $\{110\}_{g}$, with $\check{n}_{5}=0$ and $\check{n}_{4}=-\check{n}_{3} \neq 0$. As a necessary condition for $\rho(\mathrm{C})<6$ we obtain from (38):

$$
d=0 \quad \Leftrightarrow \quad \breve{v}_{3}^{2}=\check{v}_{4}^{2} .
$$

By possibly reflecting the $\mathbf{m}_{i} \mathrm{~s}$ we may assume that the $\check{v}_{i} \mathrm{~s}$ are positive. Then we obtain

$$
\rho(\mathrm{C})<6 \quad \Leftrightarrow \quad \check{v}_{3}=\check{v}_{4}, \quad \check{n}_{3}=-\frac{2 \breve{v}_{3}^{2}+\check{v}_{5}^{2}}{\check{v}_{5}} .
$$

Moreover, if $\rho(\mathbf{C})<6$ it is readily computed that the condition for having $\rho\left(\mathbf{C}^{2}\right)=1$ reads

$$
D^{45<}=0 \quad \Leftrightarrow \quad \check{n}_{3}=-5 \check{v}_{5}, \quad \check{v}_{3}=\sqrt{2} \check{v}_{5},
$$

while the condition for $\rho(\mathrm{C})=4$ follows from (B.33) and can always be solved for one of the $\check{H}_{i j}$.

In the case $\rho(\mathrm{C})=6$, the condition for $\rho\left(\mathrm{C}^{2}\right)=2$ is $D^{6<}=0$, which reads

$$
\breve{v}_{5}^{2} \breve{n}_{3}^{4}+4 \breve{v}_{3} \breve{v}_{4} \breve{v}_{5} \breve{n}_{3}^{3}-\left[\left(\frac{\breve{v}_{3}^{2}}{2}+\frac{\check{v}_{4}^{2}}{2}+\breve{v}_{5}^{2}\right)^{2}+\breve{v}_{5}^{4}-5 \breve{v}_{3}^{2} \breve{v}_{4}^{2}\right] \breve{n}_{3}^{2}+\left(\check{v}_{3}^{2}+\breve{v}_{4}^{2}+\breve{v}_{5}^{2}\right)^{3}=0
$$

Relations (B.19) and (B.42) imply that there are no solutions to this equation with $\breve{v}_{3}^{2}=\breve{v}_{4}^{2}$. However, an infinity of solutions exists: it is readily verified that there are, e.g., two real 
Table B1. Type III Weyl tensors: possible Segre types (columns) for given spin types (rows). The normal forms for $(X, x)=(\check{n}, \breve{v})$ of table 2 are used to define the spin types. The symbols - and $\mathrm{x}$ indicate that the corresponding Segre type is either not allowed or is unique, respectively. If $[(3331)]$ is the generic case (but other Segre types are allowed) this is denoted by G, where it is understood that the relations in columns 3 and 4 of the same row are not satisfied; for Segre type [(3322)] the relation in column 4 is not satisfied. If a spin type allows for a Segre type corresponding to $\rho(\mathrm{C})<6$ (columns 4-7), the Segre type is defined by the mentioned relation(s) and the negation of the remaining relations in columns $4-7$ of the same row; e.g., spin type $\{111\}_{\|}$ will be of Segre type [(32221)] if (B.37) and (B.40) hold (being equivalent to (B.41)), but not (B.39). In this manner, the relations written down for spin type $\{111\}_{\mathrm{g}}$ are the general Segre type defining relations; for more degenerate spin types these reduce to more specific conditions, as mentioned in the text and indicated here.

\begin{tabular}{lllllll}
\hline & {$[(3331)]$} & {$[(3322)]$} & {$[(33211)]$} & {$[(32221)]$} & {$[(331111)]$} & {$[(322111)]$} \\
\hline$\{110\}_{0}$ & - & - & $(\mathrm{B} .27)$ & - & $(\mathrm{B} .28)$ & - \\
$\{(11) 1\}_{0}$ & $\mathrm{X}$ & - & - & - & - & - \\
$\{111\}_{0}$ & $\mathrm{X}$ & - & - & - & - & - \\
$\{(000)\}_{\|}$ & $\mathrm{X}$ & - & - & - & - & - \\
$\{(11) 1\}_{\|}$ & $\mathrm{G}$ & $(\mathrm{B} .40)$ & - & - & - & - \\
$\{110\}_{\| 1}$ & $\mathrm{G}$ & $(\mathrm{B} .40)$ & - & - & $(\mathrm{B} .36),(\mathrm{B} .39)$ & - \\
$\{110\}_{\|}$ & $\mathrm{G}$ & - & $(\mathrm{B} .36)$ & - & $(\mathrm{B} .37),(\mathrm{B} .39)$ & $(\mathrm{B} .37),(\mathrm{B} .39),(\mathrm{B} .40)$ \\
$\{111\}_{\|}$ & $\mathrm{G}$ & $(\mathrm{B} .40)$ & $(\mathrm{B} .37)$ & $(\mathrm{B} .37),(\mathrm{B} .40)$ & $(\mathrm{B} .38),(\mathrm{B} .39)$ & - \\
$\{(11) 1\}_{\perp}$ & $\mathrm{G}$ & $(\mathrm{B} .40)$ & $(\mathrm{B} .38)$ & - & - & - \\
$\{111 / 0\}_{\perp}$ & $\mathrm{G}$ & $D^{6<}=0$ & - & - & - & - \\
$\{(11) 1\}_{\mathrm{g}}$ & $\mathrm{G}$ & $D^{6<}=0$ & - & - & $(\mathrm{B} .43), D^{45}=0$ & $(\mathrm{~B} .43),(\mathrm{B} .44), D^{45}=0$ \\
$\{110\}_{\mathrm{g}}$ & $\mathrm{G}$ & $(\mathrm{B} .45)$ & $(\mathrm{B} .43)$ & $(\mathrm{B} .43),(\mathrm{B} .44)$ & $\left.D^{6}\right)$ \\
$\{111\}_{\mathrm{g}}$ & $\mathrm{G}$ & $D^{6<}=0$ & $D^{6}=0$ & $D^{6}=D^{6<}=0$ & $D^{6}=D^{45=0}$ & $D^{6}=D^{6<}=D^{45}=0$ \\
\hline
\end{tabular}

solutions $\check{n}_{3} / \check{v}_{5}$ when $\check{v}_{3}=\check{v}_{5}$ and $\check{v}_{4}=3 \check{v}_{5}$. We conclude that for spin type III $\{110\}_{\mathrm{g}}$ all six Segre types of table 5 are possible.

The same conclusion is valid, a fortiori, for III $\{111\}_{\mathrm{g}}$. Note from (38) that $\check{v}_{3}=\check{v}_{4}=\check{v}_{5}$ implies $d=\breve{n}_{3} \check{n}_{4} \breve{n}_{5} \neq 0$ (recall $\check{n}_{3}+\check{n}_{4}+\check{n}_{5}=0$ ) and thus $\rho(\mathrm{C})=6$; there are, e.g., two real solutions $\check{n}_{3} / \check{v}_{5}$ to $D^{6<}=0$ for the subcase $\check{n}_{4}=2 \check{n}_{3}$ thereof. For $\check{v}_{3}=\check{v}_{4}\left(\neq \pm \check{v}_{5}\right)$, we have that

$$
\rho(\mathrm{C})<6 \quad \Leftrightarrow \quad \check{n}_{3}-\check{n}_{4}=3 \check{v}_{5}, \quad \check{n}_{5}= \pm \sqrt{4 \check{v}_{3}^{2}+5 \check{v}_{5}^{2}}
$$

while $D^{45<}=0$ if, in addition, $\breve{v}_{5}^{2}=7 \breve{v}_{3}^{2}$.

Table B1 summarizes the relation between the spin type and Segre type refinement schemes for 5D alignment type III Weyl tensors.

\section{Appendix C. Spin types versus eigenvalues for Weyl type II}

In this section we shall illustrate the classification of a type II Weyl operator based on its spin type. We shall indicate the degeneracies in the eigenvalue spectra of $\mathrm{M}, \Omega$ and $\mathrm{C}$, and indicate a zero eigenvalue by 0 . So, for instance, $C\{(000)(1111)(11) 1\}$ indicates that $C$ has one single, one double and one quadruple non-zero eigenvalue, while 0 is a triple eigenvalue. In particular, we present the case $\bar{w}=0$ and comment on the more general situations, where the subdivision is based on the number of zero components in the normal forms of table 3 .

\section{C.1. Case $\bar{w}=0$}

The spin type can be any of the $\{\cdots\}_{0}$ types. The matrices $M$ and $\Omega$ are diagonal so that their eigenvalues can be immediately read off; namely $\left[-\bar{R}_{3} / 2,-\bar{R}_{4} / 2,-\bar{R}_{5} / 2\right]$ for $M$ and 
$\left[\bar{R} / 2, \bar{R} / 2-\bar{R}_{3}, \bar{R} / 2-\bar{R}_{4}, \bar{R} / 2-\bar{R}_{5}\right]$ for $\Omega$. Obviously, none of these can be nilpotent in this case (unless they vanish identically, leading to type III spacetimes). We also observe that the combination $\rho(M)=2, \rho(\Omega)=3$ is not permitted here. Let us remark that, in the case of type $\mathbf{D}$, the presently considered case $\bar{w}=0$ is of some interest since it defines a purely electric type D Weyl tensor-several properties and explicit examples of such spacetimes are known [25] (see also section 7).

$\{(000)\}_{0}[\bar{R} \neq 0]$. This type arises when $\bar{R}_{3}=\bar{R}_{4}=\bar{R}_{5}=\bar{R} / 3 \neq 0$. Clearly we have $M:\{(111)\}$ (or $M:\{-\bar{R} / 6[3]\}), \Omega:\{(111) 1\}$ (or $\Omega:\{\bar{R} / 6[3], \bar{R} / 2[1]\}$ ), so that

$$
C:\{(111111)(111) 1\}
$$

or $C:\{-\bar{R} / 6[6], \bar{R} / 6[3], \bar{R} / 2[1]\}$. We observe that in the case of type $\mathbf{D}$ this is the spin type of the five-dimensional Schwarzschild-Tangherlini solution (cf subsection 6.4 of [14]) and, more generally, of any Robinson-Trautman spacetime (not necessarily empty) [26].

$\{(11) 1\}_{0}[\bar{R} \neq 0]$. This occurs for $\bar{R}_{5}=\bar{R}_{4}$ (up to permutations of the axes). In general we have $M:\{(11) 1\}, \Omega:\{(11) 11\}$, so that

$$
C:\{(1111)(11)(11) 11\} \text {. }
$$

It is interesting to observe that, for type $\mathbf{D}$ Einstein spacetimes, the presence of a non-geodetic multiple WAND is equivalent to the spacetime having this spin type, with $\bar{R}_{5}=\bar{R}_{4}=-\bar{R}_{3}$ (see proposition 9 in [14] and [16, 27]). All such spacetimes have in fact been found in [27], where it was also shown that in any dimensions an Einstein spacetime with a non-geodetic multiple WAND must be of type $\mathbf{D}$.

If $\bar{R}_{3}=2 \bar{R}_{4}$ the second (non-degenerate) eigenvalue of $\Omega$ turns into 0 , so that $C:\{(1111)(11)(11) 10\}$. There may be more special types in the following cases.

(1) For $\bar{R}_{3}=0, M:\{(11) 0\}(M:\{-\bar{R} / 4[2], 0[1]\}), \Omega:\{(11)(00)\}(\Omega:\{\bar{R} / 2[2], 0[2]\})$, so that

$$
C:\{(1111)(0000)(11)\}
$$

In the case of type $\mathbf{D}$ this is, in particular, the spin type of Schwarzschild black strings (and Kerr black strings on the equatorial plane).

(2) For $\bar{R}_{4}=0, M:\{(00) 1\}(M:\{0[2],-\bar{R} / 2[1]\}), \Omega:\{(111) 1\}(\Omega:\{\bar{R} / 2[3],-\bar{R} / 2[1]\})$, so that

$$
C:\{(0000)(111)(111)\}
$$

or $C:\{0[4], \bar{R} / 2[3],-\bar{R} / 2[3]\}$.

$\{(11) 1\}_{0}[\bar{R}=0]$. This can be seen as a subcase of the previous spin type, and occurs for $\bar{R}_{5}=\bar{R}_{4}, \bar{R}_{3}=-2 \bar{R}_{4}$. We find $M:\{(11) 1\}$ and $\Omega:\{(11) 10\}$, so that

$$
C:\{(1111)(11)(11) 10\} \text {. }
$$

$\{111 / 0\}_{0}[\bar{R} \neq 0]$. This is the case of a generic matrix $\bar{S}$, and in general we have $M:\{111\}$, $\Omega:\{1111\}$ and hence

$$
C:\{(11)(11)(11) 1111\} .
$$

A single eigenvalue of $\Omega$ can be zero for special values of the $\bar{R}_{i}$ (e.g., for $\bar{R}_{5}=\bar{R}_{3}+\bar{R}_{4}$; the case $\bar{R}_{5}=-\bar{R}_{3}-\bar{R}_{4}$ corresponds to $\bar{R}=0$ and is thus discussed separately below).

Further degeneracies in the spectrum of $C$ can still occur when $\Omega$ has degenerate eigenvalues, or when $M$ and $\Omega$ have some common eigenvalues, as we now discuss. 
(1) $\Omega$ has a degenerate eigenvalue iff $\bar{R}_{5}=0$ (up to axis permutation) so that $M:\{110\}$, $\Omega:\{(11) 11\}\left(M:\left\{-\bar{R}_{3} / 2[1],-\bar{R}_{4} / 2[1], 0[1]\right\}\right.$ and $\Omega:\left\{\bar{R} / 2[2],\left(\bar{R}_{3}-\bar{R}_{4}\right) / 2[1]\right.$, $\left.\left.\left(\bar{R}_{4}-\bar{R}_{3}\right) / 2[1]\right\}\right)$ and

$$
C:\{(11)(11)(11)(00) 11\} \text {. }
$$

(2) For $\bar{R}_{5}=-\bar{R}_{4}, M:\{111\}$ and $\Omega:\{1111\}$ (more precisely, $M$ : $\left\{-\bar{R}_{3} / 2[1],-\bar{R}_{4} / 2[1], \bar{R}_{4} / 2[1]\right\}$ and $\Omega:\left\{\bar{R}_{3} / 2[1],-\bar{R}_{3} / 2[1],\left(\bar{R}_{3} / 2-\bar{R}_{4}\right)[1],\left(\bar{R}_{3} / 2+\right.\right.$ $\left.\left.\left.\bar{R}_{4}\right)[1]\right\}\right)$, so that

$$
C:\{(111)(11)(11) 111\} .
$$

(3) For $\bar{R}_{5}=-\left(\bar{R}_{3}+\bar{R}_{4}\right) / 2, M:\{111\}$ and $\Omega:\{1111\}$ (more precisely, $M$ : $\left\{-\bar{R}_{3} / 2[1],-\bar{R}_{4} / 2[1],\left(\bar{R}_{3}+\bar{R}_{4}\right) / 4[1]\right\}$ and $\Omega:\left\{\left(\bar{R}_{3}+\bar{R}_{4}\right) / 4[1],\left(\bar{R}_{4}-3 \bar{R}_{3}\right) / 4[1],\left(\bar{R}_{3}-\right.\right.$ $\left.\left.\left.3 \bar{R}_{4}\right) / 4[1], 3\left(\bar{R}_{3}+\bar{R}_{4}\right) / 4[1]\right)\right)$, so that, again

$$
C:\{(111)(11)(11) 111\} \text {. }
$$

(4) For $\bar{R}_{5}=\left(\bar{R}_{3}-\bar{R}_{4}\right) / 2, M:\{111\}$ and $\Omega:\{1111\}$ (more precisely, $M:\left\{-\bar{R}_{3} / 2[1],-\bar{R}_{4} / 2[1],\left(-\bar{R}_{3}+\bar{R}_{4}\right) / 4[1]\right\}$ and $\Omega:\left\{\left(3 \bar{R}_{3}+\bar{R}_{4}\right) / 4[1],\left(\bar{R}_{4}-\right.\right.$ $\left.\left.\left.\bar{R}_{3}\right) / 4[1], 3\left(\bar{R}_{3}-\bar{R}_{4}\right) / 4[1],\left(\bar{R}_{3}+3 \bar{R}_{4}\right) / 4[1]\right)\right)$, and again

$$
C:\{(111)(11)(11) 111\} \text {. }
$$

$\{111\}_{0}[\bar{R}=0]$. This case arises for $\bar{R}_{5}=-\bar{R}_{3}-\bar{R}_{4}$. In general $M$ and $\Omega$ have no common eigenvalues, and $\Omega$ has no multiple eigenvalues, so that

$$
C:\{(11)(11)(11) 1110\} \text {. }
$$

However, if $\bar{R}_{4}=-\bar{R}_{3}$ then $M:\{110\}\left(M:\left\{-\bar{R}_{3} / 2[1], \bar{R}_{3} / 2[1], 0[1]\right\}\right)$ and $\Omega:\{(00) 11\}$ $\left(\Omega:\left\{0[2],-\bar{R}_{3}[1], \bar{R}_{3}[1]\right)\right)$, and hence

$$
C:\{(0000)(11)(11) 11\} \text {. }
$$

\section{C.2. Case $\bar{w}_{3} \neq 0=\bar{w}_{4}=\bar{w}_{5}$}

It can easily be seen that the combination $\rho(M)=2, \rho(\Omega)=3$ is still not permitted here, and $\Omega$ cannot be nilpotent.

$\{111 / 0\}_{\|}[\bar{R} \neq 0]$. This is the spin type defined by the conditions $\bar{w}_{3} \neq 0=\bar{w}_{4}=\bar{w}_{5}$ when no further restrictions apply (special subtypes are discussed in detail below). It is generically $\{111\}_{\|}$, although also type $\{110\}_{\|}$shows up in special instances described below. While we focus here mostly on the case $\bar{R} \neq 0$, some special subcases with $\bar{R}=0$ will also be mentioned, when they arise naturally.

The eigenvalues of $M$ and $\Omega$ are

$\lambda_{M}=\left\{-\frac{\bar{R}_{3}}{2}, \frac{1}{4}\left(-\bar{R}_{4}-\bar{R}_{5} \pm \sqrt{\left(\bar{R}_{4}-\bar{R}_{5}\right)^{2}-4 \bar{w}_{3}^{2}}\right)\right\}$

$\lambda_{\Omega}=\left\{\frac{1}{2}\left(\bar{R}_{3}+\bar{R}_{4}-\bar{R}_{5}\right), \frac{1}{2}\left(\bar{R}_{3}-\bar{R}_{4}+\bar{R}_{5}\right), \frac{1}{2}\left(\bar{R}_{4}+\bar{R}_{5} \pm \sqrt{\bar{R}_{3}^{2}-4 \bar{w}_{3}^{2}}\right)\right\}$.

Let us remark that our analysis is always restricted to Weyl tensors with real components only. Note, however, that in certain ranges of the parameters $M$ and $\Omega$ may admit a pair of complex conjugate eigenvectors. When that happens the corresponding pair $\{11\}$ should be replaced by $\{X \bar{X}\}$. For brevity, we shall sometimes omit this distinction at intermediate steps (whenever it depends on the value of an arbitrary parameter), and we shall be fully explicit 
only in the summarizing results for the eigenvalue structure of $C$. Thus, with these conventions we generically have $M:\{111\}$ and $\Omega:\{1111\}$.

An eigenvalue of $M$ vanishes for $\bar{R}_{3}=0$ or $\bar{w}_{3}^{2}=-\bar{R}_{4} \bar{R}_{5}$, while $\Omega$ has a zero eigenvalue for $\bar{R}_{5}= \pm \bar{R}_{3}+\bar{R}_{4}$. It easily follows that $M$ is nilpotent (with $M^{2}=0$ ) iff $\bar{R}_{3}=0=\bar{R}_{4}+\bar{R}_{5}$ (so that $\bar{R}=0$ ) with $\bar{w}_{3}^{2}=\bar{R}_{4}^{2}$, corresponding to a subcase of the case $\rho(M)=1, \rho(\Omega)=4$, cf (84) (up to a trivial axis permutation). As already mentioned, $\Omega$ can not be of type $\{(0000)\}$ here. Possible repeated eigenvalues are now discussed.

(1) For $2 \bar{w}_{3}=\bar{R}_{4}-\bar{R}_{5}, M:\{(11) 1\}, \Omega:\{1111\}$, and

$$
C:\{(1111)(11) 1111\} \text {, }
$$

where the last pair becomes $\{X \bar{X}\}$ if $\left(\bar{R}_{4}-\bar{R}_{5}\right)^{2}>\bar{R}_{3}^{2}$.

(a) If, additionally, $2 \bar{R}_{3}=\bar{R}_{4}+\bar{R}_{5}$ then $M:\{(111)\}, \Omega:\{1111\}$, and

$$
C:\{(111111) 1111\}
$$

where the last pair becomes $\{X \bar{X}\}$ if $\left(\bar{R}_{4}-3 \bar{R}_{5}\right)\left(\bar{R}_{5}-3 \bar{R}_{4}\right)<0$. We observe that the spin type becomes $\{110\}_{\|}[\bar{R} \neq 0]$ here. Note also that for $\bar{R}_{5}=-\bar{R}_{4}$ one gets that $M:\{(000)\}$ is nilpotent (as already mentioned above), $\Omega:\{11 X \bar{X}\}$ and $C:\{(000000) 11 X \bar{X}\}\}$ (with $\bar{R}=0$ in this case).

(i) With the further condition $\bar{R}_{5}=3 \bar{R}_{4}$ one gets $M:\left\{-\bar{R}_{4}[3]\right\}, \Omega:\left\{2 \bar{R}_{4}[3], 0\right\}$, so that

$$
C:\{(11111)(111) 0\} \text {. }
$$

(ii) If, instead, $\bar{R}_{5}=0$ one gets $M:\{(111)\}, \Omega:\{11 X \bar{X}\}$ with one common eigenvalue, hence

$$
C:\{(1111111) 1 X \bar{X}\} \text {. }
$$

(b) If $4 \bar{R}_{3} \bar{R}_{4}=5 \bar{R}_{4}^{2}-2 \bar{R}_{4} \bar{R}_{5}+\bar{R}_{5}^{2}$ then $M:\{(11) 1\}, \Omega:\{(11) 11\}$, therefore

$$
C:\{(1111)(11)(11) 11\} \text {. }
$$

(i) If $\bar{R}_{5}=-3 \bar{R}_{4}$ then $M$ and $\Omega$ share their double eigenvalue and one single eigenvalue, so that

$$
C:\{(111111)(111) 1\} .
$$

The spin type is $\{110\}_{\|}[\bar{R} \neq 0]$ here.

(ii) If $\bar{R}_{5}=9 \bar{R}_{4}$, the double $M$-eigenvalue equals a single $\Omega$-eigenvalue, hence

$$
C:\{(11111)(11)(11) 1\} \text {. }
$$

The spin type is $\{110\}_{\|}[\bar{R} \neq 0]$ here.

(c) If $\bar{R}_{3}=\bar{R}_{4}-\bar{R}_{5}$ then $M:\{(11) 1\}, \Omega:\{(11) 10\}$, so that

$$
C:\{(1111)(11)(11) 10\} \text {. }
$$

(i) If $\bar{R}_{5}=-\bar{R}_{4}$ the double eigenvalues of $M$ and $\Omega$ vanish, thus giving

$$
C:\{(0000000)(11) 1\} \text {. }
$$

(ii) If $\bar{R}_{4}=0$ the double eigenvalue of $\Omega$ equals the single eigenvalue of $M$, therefore

$$
C:\{(1111)(1111) 10\} \text {. }
$$

The spin type is $\{110\}_{\|}[\bar{R}=0]$.

(iii) If $3 \bar{R}_{5}=5 \bar{R}_{4}$ the single non-zero eigenvalue of $\Omega$ equals the double eigenvalue of $M$, which implies

$$
C:\{(11111)(11)(11) 0\} \text {. }
$$

(d) If $2 \bar{R}_{3}=\bar{R}_{4}-\bar{R}_{5}, M:\{(11) 1\}, \Omega:\{11 X \bar{X}\}$, and the single eigenvalue of $M$ is also an eigenvalue of $\Omega$, hence

$$
C:\{(1111)(111) 1 X \bar{X}\}
$$

which reduces to $C:\{(0000)(111) 1 X \bar{X}\}$ if $\bar{R}_{5}=-\bar{R}_{4}$. 
When, additionally, $\bar{R}_{5}=2 \bar{R}_{4}$, then also the double eigenvalue of $M$ is an eigenvalue of $\Omega$, so that

$$
C:\{(11111)(111) X \bar{X}\} .
$$

(e) If $\left(\bar{R}_{4}+\bar{R}_{5}\right) \bar{R}_{3}=-\left(\bar{R}_{4}^{2}+\bar{R}_{5}^{2}\right), M:\{(11) 1\}, \Omega:\{1111\}$, and the single eigenvalue of $M$ is also an eigenvalue of $\Omega$, hence

$$
C:\{(1111)(111) 111\} \text {. }
$$

(f) If $2 \bar{R}_{3}=\bar{R}_{4}-3 \bar{R}_{5}, M:\{(11) 1\}, \Omega:\{1111\}$, and the double eigenvalue of $M$ is also an eigenvalue of $\Omega$, hence

$$
C:\{(11111)(11) 111\},
$$

where the last pair becomes $\{X \bar{X}\}$ if $\left(\bar{R}_{4}+\bar{R}_{5}\right)\left(5 \bar{R}_{5}-3 \bar{R}_{4}\right)<0$.

(g) If $2 \bar{R}_{3}= \pm \sqrt{13\left(\bar{R}_{4}^{2}+\bar{R}_{5}^{2}\right)+10 \bar{R}_{4} \bar{R}_{5}}, M:\{(11) 1\}, \Omega:\{1111\}$, and the double eigenvalue of $M$ is also an eigenvalue of $\Omega$, hence

$$
C:\{(11111)(11) 111\} \text {. }
$$

(2) For $\bar{w}_{3}^{2}=-\left(\bar{R}_{3}-\bar{R}_{4}\right)\left(\bar{R}_{3}-\bar{R}_{5}\right), M:\{(11) 1\}, \Omega:\{1111\}$, and

$$
C:\{(1111)(11) 1111\} \text {, }
$$

where the last pair becomes $\{X \bar{X}\}$ if $\bar{R}_{3}^{2}+4\left(\bar{R}_{3}-\bar{R}_{4}\right)\left(\bar{R}_{3}-\bar{R}_{5}\right)<0$. Note also that $F_{3}^{*}=0$ identically here, therefore the spin type is $\{111\}_{\|}[\bar{R} \neq 0]$ (including all the following special subcases unless stated otherwise). For $\bar{R}_{3}=0$ (so that $\bar{w}_{3}^{2}=-\bar{R}_{4} \bar{R}_{5}$ ) one gets $M:\{(00) 1\}, \Omega:\{11 X \bar{X}\}$ and $C:\{(0000)(11) 11 X \bar{X}\}$, which describes the case $\rho(M)=1, \rho(\Omega)=4$ of $(84)$ (the more special subcase $M:\{(000)\}$ has been already mentioned).

(a) If $\bar{R}_{3}=\bar{R}_{4}-\bar{R}_{5}, M:\{(11) 1\}, \Omega:\{(11) 10\}$, hence

$C:\{(1111)(11)(11) 10\}$.

(b) If $4 \bar{R}_{5}\left(\bar{R}_{3}-\bar{R}_{4}\right)=\bar{R}_{3}\left(5 \bar{R}_{3}-4 \bar{R}_{4}\right), M:\{(11) 1\}, \Omega:\{(11) 11\}$, hence

$$
C:\{(1111)(11)(11) 11\} \text {. }
$$

(i) If, in addition, $\sqrt{3} \bar{R}_{3}= \pm 2 \bar{R}_{4}$, the double $M$-eigenvalue coincides with one of the single $\Omega$-eigenvalues, so that

$$
C:\{(11111)(11)(11) 1\} .
$$

(ii) If $9 \bar{R}_{3}=2(1 \pm \sqrt{10}) \bar{R}_{4}$, the double $M$-eigenvalue coincides with the double $\Omega$-eigenvalue, so that

$$
C:\{(111111)(11) 11\} \text {. }
$$

The spin type is $\{111\}_{\|}[\bar{R}=0]$.

(iii) If $\bar{R}_{4}=0$, the single $M$-eigenvalue coincides with one of the single $\Omega$-eigenvalues, therefore

$$
C:\{(1111)(111)(11) 1\} .
$$

(iv) If $3 \bar{R}_{3}=(-1 \pm \sqrt{13}) \bar{R}_{4}$, the single $M$-eigenvalue coincides with the double $\Omega$-eigenvalue, which implies

$$
C:\{(1111)(1111) 11\} \text {. }
$$

(c) If $2 \bar{R}_{3}=\bar{R}_{5}-\bar{R}_{4}, M:\{(11) 1\}, \Omega:\{1111\}$, where the double $M$-eigenvalue is also an $\Omega$-eigenvalue, so that

$$
C:\{(11111)(11) 111\},
$$

where the last pair becomes $\{X \bar{X}\}$ if $13 \bar{R}_{4}+6 \bar{R}_{4} \bar{R}_{5}-3 \bar{R}_{5}<0$ (with $\bar{w}_{3}^{2}>0$ ).

(d) Also for $4 \bar{R}_{3}=3\left(\bar{R}_{4}+\bar{R}_{5}\right) \pm \sqrt{13 \bar{R}_{4}+10 \bar{R}_{4} \bar{R}_{5}+13 \bar{R}_{5}}, M:\{(11) 1\}, \Omega:\{1111\}$, with the double $M$-eigenvalue being also an $\Omega$-eigenvalue, so that

$$
C:\{(1111)(11) 111\} \text {. }
$$


(e) If $\bar{R}_{4}=0, M:\{(11) 1\}, \Omega:\{1111\}$, now sharing the single $M$-eigenvalue, i.e.

$$
C:\{(1111)(111) 111\}
$$

where the last pair becomes $\{X \bar{X}\}$ if $\bar{R}_{3}\left(5 \bar{R}_{3}-4 \bar{R}_{5}\right)<0$ (with $\left.\bar{w}_{3}^{2}>0\right)$.

(f) If $\bar{R}_{3}= \pm \sqrt{\bar{R}_{4}^{2}+\bar{R}_{4} \bar{R}_{5}+\bar{R}_{5}^{2}}, M:\{(11) 1\}$ and $\Omega:\{1111\}$ again share the single $M$-eigenvalue, thus

$$
C:\{(1111)(111) 111\} \text {. }
$$

(3) For $4 \bar{w}_{3}^{2}=\bar{R}_{3}^{2}, M:\{111\}, \Omega:\{(11) 11\}$. Further possible subcases can easily be studied as illustrated above, and listing all possibilities is not particularly illuminating.

(4) For $\bar{w}_{3}^{2}=\bar{R}_{4}\left(\bar{R}_{3}-\bar{R}_{4}\right)$, one has again $M:\{111\}, \Omega:\{(11) 11\}$.

(5) There are four possible choices of parameters corresponding to $M$ and $\Omega$ having a common eigenvalue (which is thus a multiple eigenvalue of $C$ ). These are the cases (the common roots $x_{0}$ are indicated between square brackets):
(a) $\bar{w}_{3}^{2}=-\left(\bar{R}_{3}+\bar{R}_{5}\right)\left(\bar{R}_{3}-\bar{R}_{4}+2 \bar{R}_{5}\right) \quad\left[2 x_{0}=\bar{R}_{3}+\bar{R}_{5}-\bar{R}_{4}\right]$
(b) $4 \bar{w}_{3}^{2}=-\left(\bar{R}_{4}+\bar{R}_{5}\right)\left(2 \bar{R}_{3}+\bar{R}_{4}+\bar{R}_{5}\right)$;
(c) $3 \bar{w}_{3}^{2}=\bar{R}_{3}^{2}-4\left(\bar{R}_{4}^{2}+\bar{R}_{5}^{2}\right)-7 \bar{R}_{4} \bar{R}_{5} \pm\left(\bar{R}_{4}+\bar{R}_{5}\right) \sqrt{D}, \quad D \equiv 3\left(4 \bar{R}_{4}^{2}+4 \bar{R}_{5}^{2}+4 \bar{R}_{4} \bar{R}_{5}-\bar{R}_{3}^{2}\right)$;
(d) $2 \bar{R}_{3}=\bar{R}_{4}-\bar{R}_{5}$.

The common roots $x_{1}$ are given by: (a) $2 x_{1}=\bar{R}_{3}+\bar{R}_{5}-\bar{R}_{4}$, (b) and (d) $2 x_{1}=\bar{R}_{3}$, (c) $x_{1}=-\left(\bar{R}_{4}+\bar{R}_{5}\right) / 2+\sqrt{D} / 6$. Further specializations may give more common roots; for instance, if $\bar{R}_{4}=-\bar{R}_{5}$ additionally holds in case (c), such that the type becomes $\{110\}_{\|}$, then $x_{2}=-\sqrt{D} / 6$ is another common root. In general $M:\{111\}, \Omega:\{1111\}$ in all the above cases, but some degeneracy is possible in special subcases.

$\{111 / 0\}_{\|}[\bar{R}=0]$. This case is analogous to the case $\{111 / 0\}_{\|}[\bar{R} \neq 0]$ discussed above, and can be analyzed similarly with the additional condition $\bar{R}=0$. Some special subcases have been already mentioned above.

$\{(000)\}_{\|}[\bar{R} \neq 0]$. Here we have $\bar{R}_{3}=\bar{R}_{4}=\bar{R}_{5}=\bar{R} / 3 \neq 0$. It follows from (C.13) that $M:\{1 X \bar{X}\}$ and $\Omega:\{(11) 11\}$, so that

$$
C:\{(11)(11)(X X)(\bar{X} \bar{X}) 11\}
$$

where the last pair becomes $\{Z \bar{Z}\}$ if $4 \bar{w}_{3}^{2}>\bar{R}_{3}^{2}$.

$M$ cannot have any degenerate eigenvalues. For $\bar{R}_{3}^{2}=4 \bar{w}_{3}^{2}$, however, $\Omega$ has two double eigenvalues, namely $\bar{R}_{3}$ and $\bar{R}_{3} / 2$, therefore

$$
C:\{(11)(11)(11)(X X)(\bar{X} \bar{X})\} \text {. }
$$

$\{(000)\}_{\|}[\bar{R}=0]$. If all $\bar{R}_{i}=0$ then $M:\left\{0[1], \mathrm{i} \bar{w}_{3} / 2[1],-\mathrm{i} \bar{w}_{3} / 2[1]\right\}$ and $\Omega$ : $\left\{0[2], \mathrm{i} \bar{w}_{3}[1],-\mathrm{i} \bar{w}_{3}[1]\right\}$, so that

$$
C:\{(0000)(X X)(\bar{X} \bar{X}) Z \bar{Z}\} .
$$

Observe that, in the case of type $\mathbf{D}$, this is the spin type that defines a purely magnetic type D Weyl tensor. It has recently been shown that (in any higher dimensions) such spacetimes cannot occur, if one adds also the Ricci-flat/Einstein condition [25]. So far no type D purely magnetic spacetimes are known in 5D (or in any dimension higher than 4); in 4D the only known such spacetimes are locally rotationally symmetric, see [40, 41].

$\{(11) 1)\}_{\|}[\bar{R} \neq 0]$. This spin type occurs for $\bar{R}_{5}=\bar{R}_{4}$. We observe, in particular, that in the type $\mathbf{D}$ case this is the spin type of both five-dimensional Myers-Perry black holes and Kerr black strings, and in fact of a 'generic' five-dimensional Kerr-Schild spacetime (cf subsection 6.4 of [14] and subsection 5.5 of [49]). From (C.13) one gets $M:\{1 X \bar{X}\}$ and $\Omega:\{(11) 11\}$, so that in general

$$
C:\{(11)(11)(X X)(\bar{X} \bar{X}) 11\},
$$


where the last pair becomes $\{Z \bar{Z}\}$ if $4 \bar{w}_{3}^{2}>\bar{R}_{3}^{2}$. The Myers-Perry black hole has this general eigenvalue spectrum. One of the non-degenerate eigenvalues of $\Omega$ becomes zero if $4 \bar{w}_{3}^{2}=\bar{R}_{3}^{2}-4 \bar{R}_{4}^{2}$, in which case then

$$
C:\{(11)(11)(X X)(\bar{X} \bar{X}) 10\} \text {. }
$$

The eigenvalues of $M$ can never be degenerate for this spin type. Nevertheless, further degeneracies are possible in the following cases.

(1) For $4 \bar{w}_{3}^{2}=\bar{R}_{3}^{2}, M:\{1 X \bar{X}\}, \Omega:\{(11)(11)\}$, and

$$
C:\{(11)(11)(X X)(\bar{X} \bar{X})(11)\} \text {. }
$$

This becomes even more special if one of the followings additionally holds.

(a) If $\bar{R}_{3}=2 \bar{R}_{4}$ then $M:\{1 X \bar{X}\}, \Omega:\{(1111)\}$ (or $\left.\Omega:\left\{\bar{R}_{4}[4]\right\}\right)$, and

$$
C:\{(1111)(11)(X X)(\bar{X} \bar{X})\} .
$$

(b) If $\bar{R}_{4}=0$ then $M:\{0 X \bar{X}\}, \Omega:\{(11)(00)\}$, and

$$
C:\{(0000)(11)(11)(X X)(\bar{X} \bar{X})\} \text {. }
$$

(2) For $\bar{w}_{3}^{2}=\bar{R}_{4}\left(\bar{R}_{3}-\bar{R}_{4}\right), M:\{1 X \bar{X}\}, \Omega:\{(111) 1\}$, and

$$
C:\{(111)(11)(X X)(\bar{X} \bar{X}) 1\} .
$$

The non-degenerate eigenvalue of $\Omega$ vanishes for $\bar{R}_{3}=4 \bar{R}_{4}$, in which case

$$
C:\{(111)(11)(X X)(\bar{X} \bar{X}) 0\} .
$$

(3) For $\bar{R}_{3}=0$ we get $M:\{X \bar{X} 0\}, \Omega:\{(00) Z \bar{Z}\}$, thus

$$
C:\{(0000)(X X)(\bar{X} \bar{X}) Z \bar{Z}\} .
$$

This is the eigenvalue spectrum for the Kerr black string (away from the equatorial plane, where it is (C.3)).

(4) For $\bar{w}_{3}^{2}=-\bar{R}_{4}\left(\bar{R}_{3}+\bar{R}_{4}\right)$, we get $M:\{1 X \bar{X}\}$ and $\Omega:\{(11) 11\}$, but $M$ and $\Omega$ share one non-degenerate eigenvalue (i.e. $-\bar{R}_{3} / 2$ ), so that

$$
C:\{(111)(11)(X X)(\bar{X} \bar{X}) 1\} .
$$

The only non-degenerate eigenvalue of $C$ vanishes for $\bar{R}_{3}=-4 \bar{R}_{4}$, in which case

$$
C:\{(111)(11)(X X)(\bar{X} \bar{X}) 0\} .
$$

(5) For $\bar{R}_{4}=0=\bar{R}_{3}^{2}-3 \bar{w}_{3}^{2}$, we have $M:\{1 X \bar{X}\}$ and $\Omega:\{(11) X \bar{X}\}$, so that

$$
C:\{(X X X)(\bar{X} \bar{X} \bar{X})(11)(11)\} \text {. }
$$

$\{(11) 1)\}_{\|}[\bar{R}=0]$. This can also be understood as a subcase of the previous spin type, with again $\bar{R}_{5}=\bar{R}_{4}$, and the additional condition $\bar{R}_{3}=-2 \bar{R}_{4}$. Similarly, we have in general $M:\{1 X \bar{X}\}$ and $\Omega:\{(11) 11\}$, which gives

$$
C:\{(11)(11)(X X)(\bar{X} \bar{X}) 11\},
$$

where the last pair becomes $\{Z \bar{Z}\}$ if $\bar{w}_{3}^{2}>\bar{R}_{4}^{2}$. No eigenvalues can vanish here.

The only case with further degeneracy now arises for $\bar{w}_{3}^{2}=\bar{R}_{4}^{2}$, giving $M:\{1 X \bar{X}\}$ and $\Omega:\{(11)(11)\}$ with a common eigenvalue (namely $\bar{R}_{4}$ ), so that

$$
C:\{(1111)(11)(X X)(\bar{X} \bar{X})\} .
$$

$\{(11) 1)\}_{\perp}[\bar{R} \neq 0]$. This is defined by $\bar{R}_{4}=\bar{R}_{3}$. Hence $M:\{111\}$ (or $M:\{1 X \bar{X}\}$ if $\left.\left(\bar{R}_{3}-\bar{R}_{5}\right)^{2}<4 \bar{w}_{3}^{2}\right)$ and $\Omega:\{1111\}$ (or $\Omega:\{11 Z \bar{Z}\}$ if $4 \bar{w}_{3}^{2}>\bar{R}_{3}^{2}$ ), so that

$$
C:\{(11)(11)(11) 1111\} \text {, }
$$


or $C:\{(11)(X X)(\bar{X} \bar{X}) 1111\}$ or $C:\{(11)(X X)(\bar{X} \bar{X}) 11 Z \bar{Z}\}$ or $C:\{(11)(11)(11) 11 Z \bar{Z}\}$ according to the specific values of the parameters. One of the $M$-eigenvalues vanishes for either $\bar{R}_{3}=0$ or $\bar{w}_{3}^{2}=-\bar{R}_{3} \bar{R}_{5}$, while $\Omega$ has a zero eigenvalue for $\bar{R}_{5}=0$ or $\bar{R}_{5}=2 \bar{R}_{3}$ or $4 \bar{w}_{3}^{2}=-\bar{R}_{5}\left(2 \bar{R}_{3}+\bar{R}_{5}\right)$.

Additional degeneracies of the eigenvalues are possible in the following cases.

(1) For $4 \bar{w}_{3}^{2}=\left(\bar{R}_{3}-\bar{R}_{5}\right)^{2} M$ has a double eigenvalue, i.e. $M:\{(11) 1\}, \Omega:\{1111\}$, and

$$
C:\{(1111)(11) 1111\} \text {, }
$$

where the last pair becomes $\{X \bar{X}\}$ if $\bar{R}_{5}\left(2 \bar{R}_{3}-\bar{R}_{5}\right)<0$. The single $M$-eigenvalue vanishes for $\bar{R}_{3}=0$, in which case then $M:\{(11) 0\}, \Omega:\{1111\}$, and $C:\{(1111)(00) 11 X \bar{X}\}$. Other $C$-eigenvalues can possibly vanish only in more degenerate cases, which are all now listed.

(a) If $\bar{R}_{3}=5 \bar{R}_{5}$ then $M:\{(11) 1\}, \Omega:\{(11) 11\}$, so that

$$
C:\{(1111)(11)(11) 11\} \text {. }
$$

(b) If $\bar{R}_{5}=0$ then $M:\{(11) 1\}, \Omega:\{(11) 10\}$, and

$$
C:\{(1111)(11)(11) 10\} \text {. }
$$

(c) If $\bar{R}_{5}=2 \bar{R}_{3}$ then $M:\{(11) 1\}, \Omega:\{(11) 10\}$, and

$$
C:\{(1111)(11)(11) 10\} .
$$

(d) If $\bar{R}_{5}=5 \bar{R}_{3}$ then $M:\{(11) 1\}, \Omega:\{11 X \bar{X}\}$, the repeated eigenvalue of $M$ (i.e. $\left.-3 \bar{R}_{3} / 2\right)$ is also an eigenvalue of $\Omega$, so that

$$
C:\{(11111)(11) 1 X \bar{X}\} .
$$

(e) If $\bar{R}_{3}=-3 \bar{R}_{5}$ then $M:\{(11) 1\}, \Omega:\{11 X \bar{X}\}$, the repeated eigenvalue of $M$ (i.e. $\left.\bar{R}_{5} / 2\right)$ is also an eigenvalue of $\Omega$, so that

$$
C:\{(11111)(11) 1 X \bar{X}\} \text {. }
$$

(f) If $\bar{R}_{5}=-\bar{R}_{3}$ then $M:\{(00) 1\}, \Omega:\{11 X \bar{X}\}$, the single eigenvalue of $M$ (i.e. $-\bar{R}_{3} / 2$ ) is also an eigenvalue of $\Omega$, so that

$$
C:\{(0000)(111) 1 X \bar{X}\} \text {. }
$$

(2) For $4 \bar{w}_{3}^{2}=\bar{R}_{3}^{2}$ it is $\Omega$ that has a double (real) eigenvalue, i.e. $M:\{111\}, \Omega:\{(11) 11\}$, and

$$
C:\{(11)(11)(11)(11) 11\},
$$

where $\{(11)(11)\}$ becomes $\{(X X)(\bar{X} \bar{X})\}$ if $\bar{R}_{5}\left(\bar{R}_{5}-\bar{R}_{3}\right)<0$. One eigenvalue of $M$ vanishes if $\bar{R}_{3}=-4 \bar{R}_{5}$, so that $C:\{(11)(11)(00)(11) 11\}$. The vanishing of a single $\Omega$ eigenvalue leads to $M$ having a double eigenvalue and it has been thus already discussed above, while the vanishing of the double eigenvalue of $\Omega$ leads to $M$ and $\Omega$ sharing a single eigenvalue and is discussed below.

Further degeneracy (omitting the already discussed cases $M:\{(11) 1\}$ ) is possible when other $\Omega$-eigenvalues coincide, or when $\Omega$ and $M$ have some common eigenvalues, as we now analyze.

(a) If $\bar{R}_{3}=2 \bar{R}_{5}$ then $M:\{1 X \bar{X}\}, \Omega:\{(111) 1\}$, so that

$$
C:\{(111)(11)(X X)(\bar{X} \bar{X}) 1\} \text {. }
$$

(b) If $\bar{R}_{5}=3 \bar{R}_{3}$ or $8 \bar{R}_{5}=25 \bar{R}_{3}$ or $4 \bar{R}_{5}=(-2 \pm \sqrt{2}) \bar{R}_{3}$, a single eigenvalue of $\Omega$ is also an eigenvalue of $M$, so that $M:\{111\}, \Omega:\{(11) 11\}$, with

$$
C:\{(111)(11)(11)(11)(11) 1\} .
$$


(c) Also for $\bar{R}_{5}=-\bar{R}_{3}$ a single eigenvalue of $\Omega$ is also an eigenvalue of $M$, but additionally the double eigenvalue of $\Omega$ vanishes, so that $M:\{111\}, \Omega:\{(00) 11\}$ and

$$
C:\{(111)(11)(11)(00) 1\} \text {. }
$$

(d) If $\bar{R}_{5}=-2 \bar{R}_{3}$ or $4 \bar{R}_{5}=(-5 \pm \sqrt{7}) \bar{R}_{3}$, the double eigenvalue of $\Omega$ is also an eigenvalue of $M$, so that $M:\{111\}, \Omega:\{(11) 11\}$, with

$$
C:\{(1111)(11)(11) 11\} \text {. }
$$

(3) Also for $\bar{w}_{3}^{2}=\bar{R}_{5}\left(\bar{R}_{3}-\bar{R}_{5}\right)$ we have $M:\{111\}, \Omega:\{(11) 11\}$ with

$$
C:\{(11)(11)(11)(11) 11\},
$$

where $\{(11)(11)\}$ becomes $\{(X X)(\bar{X} \bar{X})\}$ if $\left(\bar{R}_{3}-\bar{R}_{5}\right)\left(\bar{R}_{3}-5 \bar{R}_{5}\right)<0$. No eigenvalue can vanish here.

For $\bar{R}_{3}=2 \bar{R}_{5}$ this further specializes to $M:\{1 X \bar{X}\}, \Omega:\{(111) 1\}$ with

$$
C:\{(111)(11)(X X)(\bar{X} \bar{X}) 1\} .
$$

(4) For $\bar{R}_{5}=3 \bar{R}_{3}$ or $\bar{R}_{5}=-\bar{R}_{3}$ or $\bar{w}_{3}^{2}=2 \bar{R}_{3}\left(\bar{R}_{5}-3 \bar{R}_{3}\right), M$ and $\Omega$ are of general Segre type, however they share one eigenvalue, so that

$$
C:\{(111)(11)(11) 111\} \text {. }
$$

No eigenvalue can vanish here (unless one allows for more special cases, see the appropriate paragraphs). Some eigenvalues can be complex, in which case one should use the more precise notation $\{X \bar{X}\}$ etc, but we omit these details here.

(5) Also for $\bar{w}_{3}^{2}=-2 \bar{R}_{5}\left(\bar{R}_{3}+\bar{R}_{5}\right), M$ and $\Omega$ are of general Segre type with a common eigenvalue, and

$$
C:\{(111)(11)(11) 111\}
$$

where the last pair becomes $\{X \bar{X}\}$ if $\bar{R}_{3}^{2}+8 \bar{R}_{5}\left(\bar{R}_{3}+\bar{R}_{5}\right)<0$ (along with $\bar{w}_{3}^{2}>0$ ).

An $M$-eigenvalue vanishes for $\bar{R}_{3}=-2 \bar{R}_{5}$, hence $C:\{(111)(11)(00) X \bar{X}\}$. For $7 \bar{R}_{5}=-6 \bar{R}_{3}$ an $\Omega$-eigenvalue is zero, so that $C:\{(111)(11)(11) 110\}$.

(6) Also for $4 \bar{w}_{3}^{2}=-\left(\bar{R}_{3}+\bar{R}_{5}\right)\left(3 \bar{R}_{3}+\bar{R}_{5}\right), M$ and $\Omega$ are of general Segre type with a common eigenvalue, and

$$
C:\{(111)(11)(11) 111\} .
$$

No $M$-eigenvalue can vanish here, whereas an $\Omega$-eigenvalue is zero when $2 \bar{R}_{5}=-3 \bar{R}_{3}$, in which case $C:\{(111)(11)(11) 110\}$.

For $23 \bar{R}_{5}=-45 \bar{R}_{3}, M$ and $\Omega$ are still of generic type but they share two eigenvalues, so that

$$
C:\{(111)(111)(11) 11\}
$$

(7) The last case where $M$ and $\Omega$ are of general Segre type with a common eigenvalue arises for $3 \bar{w}_{3}^{2}=-\left(\bar{R}_{3}+\bar{R}_{5}\right)\left(3 \bar{R}_{3}+4 \bar{R}_{5} \pm \sqrt{9 \bar{R}_{3}^{2}+12 \bar{R}_{3} \bar{R}_{5}+12 \bar{R}_{5}^{2}}\right.$, with

$$
C:\{(111)(11)(11) 111\} \text {. }
$$

No $M$-eigenvalue can vanish here, whereas an $\Omega$-eigenvalue is zero when $23 \bar{R}_{5}=$ $(-25 \pm \sqrt{73}) \bar{R}_{3}$, thus giving $C:\{(111)(11)(11) 110\}$.

For $23 \bar{R}_{5}=-45 \bar{R}_{3}, M$ and $\Omega$ share two eigenvalues, but this case has been just discussed above. 
$\{(11) 1)\}_{\perp}[\bar{R}=0]$. This is defined by $\bar{R}_{4}=\bar{R}_{3}, \bar{R}_{5}=-2 \bar{R}_{3}$. Hence $M:\{111\}$ (or $M:\{1 X \bar{X}\}$ if $4 \bar{w}_{3}^{2}>9 \bar{R}_{3}^{2}$ ) and $\Omega:\{1111\}$ (or $\Omega:\{11 Z \bar{Z}\}$ if $4 \bar{w}_{3}^{2}>\bar{R}_{3}^{2}$ ), so that

$$
C:\{(11)(11)(11) 1111\},
$$

or $C:\{(11)(X X)(\bar{X} \bar{X}) 11 Z \bar{Z}\}$ or $C:\{(11)(11)(11) 11 Z \bar{Z}\}$ according to the specific values of the parameters. For $\bar{w}_{3}^{2}=2 \bar{R}_{3}^{2}$ we have $M:\{110\}$ and $\Omega:\{11 Z \bar{Z}\}$, hence $C:\{(11)(11)(00) 11 Z \bar{Z}\}$. More special cases may arise as follows.

(1) For $4 \bar{w}_{3}^{2}=9 \bar{R}_{3}^{2}, M:\{(11) 1\}, \Omega:\{11 Z \bar{Z}\}$ so that

$$
C:\{(1111)(11) 11 Z \bar{Z}\} \text {. }
$$

(2) For $4 \bar{w}_{3}^{2}=\bar{R}_{3}^{2}, M:\{111\}, \Omega:\{(11) 11\}$, and the double $\Omega$-eigenvalue is also an eigenvalue of $M$, hence

$$
C:\{(1111)(11)(11) 11\}
$$

\section{C.3. Case $\bar{w}_{4}=0 \neq \bar{w}_{3} \bar{w}_{5}$}

The possible spin types are $\{111 / 0\}_{\perp}$ and $\{(11) 1\}_{\mathrm{g}}$. Since $\bar{R}_{i}-\bar{R}_{j}=\bar{S}_{i}-\bar{S}_{j}$, at most two of the $\bar{R}_{i}$ can take the same value. In particular, we can assume that at least one $\bar{R}_{i}$ is non-zero. Note also that the combination $\rho(M)=1, \rho(\Omega)=4$ is not permitted here (cf (84)), so that, in particular, $M^{2} \neq 0$. Both $\mathrm{M}$ and $\Omega$ can be nilpotent (with $M^{2} \neq 0, \Omega^{3}=0$ ), which includes the special situation (59) when $\mathrm{C}$ is nilpotent (in which case the spin type specializes to $\{110\}_{\perp 0}[\bar{R}=0]$, cf supra).

\section{C.4. General case $\bar{w}_{3} \bar{w}_{4} \bar{w}_{5} \neq 0$}

Here all of the $\bar{R}_{i}$ take different values, otherwise (after a suitable spin) this would reduce to the previous case. Accordingly, the only possible spin type is the most general one; i.e. $\{111 / 0\} \mathrm{g}$. In particular, we can assume that at least two $\bar{R}_{i}$ are non-zero. The cases $\rho(M)=1$ (which implies $\rho(\Omega)=4$ ) and $\rho(\Omega)=2$ (which implies $\rho(M)=2$ ) are not permitted here (cf table 8). Both $M$ and $\Omega$ can be nilpotent (with $M^{2} \neq 0, \Omega^{3} \neq 0$ ), but not simultaneously.

\section{References}

[1] Coley A, Milson R, Pravda V and Pravdová A 2004 Classification of the Weyl tensor in higher dimensions Class. Quantum Grav. 21 L35-41

[2] Milson R, Coley A, Pravda V and Pravdová A 2005 Alignment and algebraically special tensors in Lorentzian geometry Int. J. Geom. Methods Mod. Phys. 2 41-61

[3] Coley A 2008 Classification of the Weyl tensor in higher dimensions and applications Class. Quantum Grav. 25033001

[4] Pravda V, Pravdová A, Coley A and Milson R 2004 Bianchi identities in higher dimensions Class. Quantum Grav. $212873-97$

See also Pravda V, Pravdová A, Coley A and Milson R 2007 Class. Quantum Grav. 241691 (corrigendum)

[5] Ortaggio M, Pravda V and Pravdová A 2007 Ricci identities in higher dimensions Class. Quantum Grav. 24 1657-64

[6] Coley A, Milson R, Pravda V and Pravdová A 2004 Vanishing scalar invariant spacetimes in higher dimensions Class. Quantum Grav. 21 5519-42

[7] Durkee M, Pravda V, Pravdová A and Reall H S 2010 Generalization of the Geroch-Held-Penrose formalism to higher dimensions Class. Quantum Grav. 27215010

[8] Stephani H, Kramer D, MacCallum M, Hoenselaers C and Herlt E 2003 Exact Solutions of Einstein's Field Equations 2nd edn (Cambridge: Cambridge University Press) 
[9] Coley A and Hervik S 2009 Higher dimensional bivectors and classification of the Weyl operator Class. Quantum Grav. 27015002

[10] Emparan R and Reall H S 2008 Black holes in higher dimensions Living Rev. Rel. 116

[11] De Smet P J 2002 Black holes on cylinders are not algebraically special Class. Quantum Grav. 19 4877-95

[12] Garcia-Parrado Gomez-Lobo A and Martin-Garcia J M 2009 Spinor calculus on 5-dimensional spacetimes J. Math. Phys. 50122504

[13] Godazgar M 2010 Spinor classification of the Weyl tensor in five dimensions Class. Quantum Grav 27245013

[14] Pravda V, Pravdová A and Ortaggio M 2007 Type D Einstein spacetimes in higher dimensions Class. Quantum Grav. 24 4407-28

[15] Ortaggio M 2009 Bel-Debever criteria for the classification of the Weyl tensor in higher dimensions Class. Quantum Grav. 26195015

[16] Garcia-Parrado Gómez-Lobo A and Wylleman L 2011 A new special class of Petrov type D vacuum space-times in dimension five J. Phys.: Conf. Ser. 314012024 (extended version in preparation)

[17] Lang S 1984 Algebra (Reading, MA: Addison-Wesley)

[18] Schell J F 1961 Classification of four-dimensional Riemannian spaces J. Math. Phys. 2 202-6

[19] Goldberg J N and Kerr R P 1961 Some applications of the infinitesimal-holonomy group to the Petrov classification of Einstein spaces J. Math. Phys. 2 327-32

[20] Ortaggio M, Pravda V and Pravdová A 2010 Type III and N Einstein spacetimes in higher dimensions: general properties Phys. Rev. D 82064043

[21] Hervik S 2011 A spacetime not characterised by its invariants is of aligned type II Class. Quantum Grav. 28215009

[22] Hervik S and Coley A 2010 Curvature operators and scalar curvature invariants Class. Quantum Grav. 27095014

[23] Yang L and Xia B 1997 Explicit criterion to determine the number of positive roots of a polynomial MM Research Preprints no $15 \mathrm{p} 134$

[24] Coley A and Hervik S 2011 Discriminating the Weyl type in higher dimensions using scalar curvature invariants Gen. Rel. Grav. 43 2199-207

[25] Hervik S, Ortaggio M and Wylleman L 2012 Minimal tensors and purely electric or magnetic spacetimes of arbitrary dimension arXiv: 1203.3563 [gr-qc]

[26] Podolský J and Ortaggio M 2006 Robinson-Trautman spacetimes in higher dimensions Class. Quantum Grav. 23 5785-97

[27] Durkee M and Reall H S 2009 A higher-dimensional generalization of the geodesic part of the Goldberg-Sachs theorem Class. Quantum Grav. 26245005

[28] Durkee M 2009 Type II Einstein spacetimes in higher dimensions Class. Quantum Grav. 26195010

[29] Pravda V and Pravdová A 2005 WANDs of the black ring Gen. Rel. Grav. 37 1277-87

[30] Godazgar M and Reall H S 2009 Algebraically special axisymmetric solutions of the higher-dimensional vacuum Einstein equation Class. Quantum Grav. 26165009

[31] Richardson R W and Slodowy P 1990 Minimum vectors for real reductive algebraic groups J. Lond. Math. Soc. 42409

[32] Hall G 1973 On the Petrov classification of gravitational fields J. Phys. A: Math. Nucl. Gen. 6619

[33] Haddow B M 1995 Purely magnetic space-times J. Math. Phys. 36 5848-54

[34] Van den Bergh N 2003 Purely gravito-magnetic vacuum spacetimes Class. Quantum Grav. 20 L1-3

[35] Van den Bergh N 2003 Tidal effects cannot be absent in a vacuum Class. Quantum Grav. 20 L165-8

[36] Wylleman L 2006 Anti-Newtonian universes do not exist Class. Quantum Grav. 23 2727-40

[37] Wylleman L and Van den Bergh N 2006 Complete classification of purely magnetic, nonrotating, nonaccelerating perfect fluids Phys. Rev. D 74084001

[38] Lozanovski C 2007 Szekeres-type mappings of Kasner and Petrov type $I\left(M^{+}\right)$purely magnetic spacetimes Class. Quantum Grav. 24 1169-88

[39] Lozanovski C and Wylleman L 2011 Complex windmill transformation producing new purely magnetic fluids Class. Quantum Grav. 28075015

[40] Lozanovski C and Carminati J 2003 Purely magnetic locally rotationally symmetric spacetimes Class. Quantum Grav. $20215-38$

[41] Van den Bergh N and Wylleman L 2006 An exhaustive classification of aligned Petrov type D purely magnetic perfect fluids Class. Quantum Grav. 23 3353-9

[42] Myers R C and Perry M J 1986 Black holes in higher dimensional space-times Ann. Phys., NY 172 304-47

[43] Emparan R and Reall H S 2002 A rotating black ring solution in five dimensions Phys. Rev. Lett. 88101101

[44] Pomeransky A A and Sen'kov R A 2006 Black ring with two angular momenta arXiv:hep-th/0612005 
[45] Elvang H and Figueras P 2007 Black saturn J. High Energy Phys. JHEP05(2007)050

[46] Elvang H and Rodriguez M J 2008 Bicycling black rings J. High Energy Phys. JHEP04(2008)045

[47] Coley A A, Hervik S, Durkee M N and Godazgar M 2011 Algebraic classification of five-dimensional spacetimes using scalar invariants Class. Quantum Grav. 28155016

[48] Lewandowski J and Pawlowski T 2005 Quasi-local rotating black holes in higher dimension: geometry Class. Quantum Grav. 22 1573-98

[49] Ortaggio M, Pravda V and Pravdová A 2009 Higher dimensional Kerr-Schild spacetimes Class. Quantum Grav. 26025008 